\title{
Nucleation and Growth of Lattice Crystals
}

\author{
Andrea Braides $^{1}$ (I) $\cdot$ Giovanni Scilla ${ }^{2} \cdot$ Antonio Tribuzio $^{1,3}$
}

Received: 2 January 2021 / Accepted: 2 August 2021 / Published online: 8 October 2021

(c) The Author(s) 2021

\begin{abstract}
A variational lattice model is proposed to define an evolution of sets from a single point (nucleation) following a criterion of "maximization" of the perimeter. At a discrete level, the evolution has a "checkerboard" structure and its shape is affected by the choice of the norm defining the dissipation term. For every choice of the scales, the convergence of the discrete scheme to a family of expanding sets with constant velocity is proved.
\end{abstract}

Keywords Discrete systems · Nucleation · Minimizing movements · Geometric evolution · Pinning $\cdot$ Microstructure

Mathematics Subject Classification 35B27 · 74Q10 · 53E10 - 49M25 · 49J45

\section{Introduction}

In this paper, we propose a variational model for nucleation and growth of a set by maximization of its perimeter through an energy-dissipation balance at fixed time step. We follow an implicit Euler scheme used by Almgren, Taylor and Wang to prove

Communicated by Irene Fonseca.

$凶$ Andrea Braides

braides@mat.uniroma2.it

Giovanni Scilla

giovanni.scilla@uniroma1.it

Antonio Tribuzio

antonio.tribuzio@uni-heidelberg.de

1 Dipartimento di Matematica, Università di Roma "Tor Vergata”, Via della Ricerca Scientifica 1, 00133 Rome, Italy

2 Dipartimento di Scienze di Base ed Applicate per l'Ingegneria (SBAI), Sapienza Università di Roma, Via A. Scarpa 16, 00169 Rome, Italy

3 Ruprecht-Karls-Universität Heidelberg, Institut für Angewandte Mathematik, Im Neuenheimer Feld 205, 69120 Heidelberg, Germany 
existence of sets moving by mean curvature by minimization of the perimeter (see Almgren et al. 1993). In that case, fixed a time step $\tau>0$, one can define iteratively the discrete orbits $E_{k}^{\tau}$ at fixed $\tau$ from an initial set $E_{0}$ as $E_{0}^{\tau}=E_{0}$ and $E_{k}^{\tau}$ as a solution of

$$
\min \left\{P(E)+\frac{1}{\tau} D^{p}\left(E, E_{k-1}^{\tau}\right)\right\}, \quad D^{p}(E, F)=\int_{E \triangle F} \operatorname{dist}_{p}(x, \partial F) \mathrm{d} x
$$

where $\operatorname{dist}_{p}(x, \partial E)=\min \left\{\|x-y\|_{p}: y \in \partial E\right\}, p \in[1, \infty]$. The term $D^{p}$ is interpreted as a dissipation, and (1.1) can be seen as a minimization of $P$ subject to a constraint due to the dissipation, which forces $E_{k}^{\tau}$ to be close to $E_{k-1}^{\tau}$ for $\tau$ small. In Almgren et al. (1993), it is proved (in the case $p=2$ ) that the piecewise-constant interpolations $E^{\tau}(t)=E_{\lfloor t / \tau\rfloor}^{\tau}$ converge to a decreasing family of sets $E(t)$ which move by mean curvature.

Such a scheme cannot be directly followed taking maximization of the perimeter as a driving mechanism, which would correspond to replacing $P$ with $-P$. Indeed, we may have sets $E$ such that $E \triangle E_{0}$ has small measure (and hence with small dissipation) but with arbitrarily large perimeter, so that the minimum value for $k=1$ in (1.1) is $-\infty$ and the scheme arrests at the first step. In order to overcome this issue, we discretize our problem by introducing a spatial length scale $\varepsilon$. For technical reasons explained below, we will examine only a two-dimensional setting and for simplicity parameterize our problem on the lattices $\varepsilon \mathbb{Z}^{2}$. We then restrict to sets that can be written as the union of squares of side length $\varepsilon$ and centers in $\varepsilon \mathbb{Z}^{2}$. Within this class, we shall consider the problem of nucleation, i.e., of motion from a minimal set, a single $\varepsilon$-square $E_{0}^{\varepsilon}$ (which we may suppose to be centered in 0 ). With fixed $\varepsilon$ and $\tau$, the discrete orbits are defined as $E_{0}^{\varepsilon, \tau}=E_{0}^{\varepsilon}$ and $E_{k}^{\varepsilon, \tau}$ as a solution of

$$
\min \left\{-P_{\varepsilon}(E)+\frac{1}{\tau} D_{\varepsilon}^{p}\left(E, E_{k-1}^{\varepsilon, \tau}\right): E_{k-1}^{\varepsilon, \tau} \subseteq E\right\}
$$

where $P_{\varepsilon}$ is the restriction of the perimeter functional to unions of $\varepsilon$-squares, and $D_{\varepsilon}^{p}$ is a discretization of the dissipation $D^{p}$ which, for every $E \supseteq F$, reduces to

$$
D_{\varepsilon}^{p}(E, F)=\varepsilon^{2} \sum_{i \in E \cap \varepsilon Z^{2}} \operatorname{dist}_{p}\left(i,\left(\varepsilon \mathbb{Z}^{2} \backslash F\right)\right) .
$$

Note that we consider a growing family of sets with respect to inclusion. With fixed $\tau=\tau_{\varepsilon}$, we will characterize the cluster points $E(t)$ as $\varepsilon \rightarrow 0$ of the interpolated functions $E^{\varepsilon}(t)=E_{\lfloor\varepsilon t / \tau\rfloor}^{\varepsilon, \tau}$, which are the generalization to varying energies of the Almgren-Taylor-Wang scheme scaled in the time variable. Note the different scaling of the time variable, which is the one that better describes the evolution. The form of $E$ will depend on the interplay between $\varepsilon$ and $\tau$; more precisely, on the limit ratio $\alpha$ of $\varepsilon^{2} / \tau$ as $\varepsilon \rightarrow 0$. We remark that the chosen time scaling can be directly interpreted as giving the minimizing movements along the sequence $-\varepsilon P_{\varepsilon}$ at scale $\tau$, which are defined in Braides (2013). This scaling is also justified by the fact that the energies $-\varepsilon P_{\varepsilon}$ have a non-trivial $\Gamma$-limit. 
We describe the case $0<\alpha<+\infty$, which is the most relevant. It is not restrictive to suppose that $\alpha \tau=\varepsilon^{2}$. By the homogeneity properties of the perimeter and the dissipation, we note that $E_{k}^{\varepsilon, \tau}=\varepsilon A_{k}^{\alpha}$, where $A_{0}^{\alpha}=q$ (the unit square centered in 0 ), and we solve iteratively

$$
\min \left\{-P_{1}(A)+\alpha D_{1}^{p}\left(A, A_{k-1}^{\alpha}\right): A_{k-1}^{\alpha} \subseteq A\right\}
$$

The first step is particularly meaningful and consists in solving the minimum problem

$$
\min \left\{-P_{1}(A)+\alpha D_{1}^{p}(A, q): q \subseteq A\right\}
$$

We have

- the first set $A_{1}^{\alpha}$ is a part of the checkerboard of unit squares in $\mathbb{R}^{2}$ containing 0 (which we call the even checkerboard). While this fact is clear "locally," the proof that the whole set is a single checkerboard requires a non-trivial covering argument, in which $\mathbb{R}^{2}$ is covered by sets in which the minimal set $A$ is (part of) the correct checkerboard. This argument can be avoided in the case $p=\infty$, which has been treated directly in Braides and Scilla (2013b);

- since every square of the (even) checkerboard gives an independent contribution of energy and dissipation, a point $i \in \mathbb{Z}^{2}$ may belong to $A_{1}^{\alpha}$ if and only if $\left(i_{1}+i_{2} \in 2 \mathbb{Z}\right.$ and) the corresponding contribution is non-positive, i.e.,

$$
-4+\alpha\|i\|_{p} \leq 0
$$

- if $\alpha \notin\left\{4 /\|i\|_{p}: i \in \mathbb{Z}^{2}, i_{1}+i_{2} \in 2 \mathbb{Z}\right\}$, then $A_{1}^{\alpha}$ is uniquely determined by (1.5), and it is the union of all squares in the even checkerboard with centers in the set

$$
\mathcal{N}_{\alpha}^{p}=\left\{i \in \mathbb{Z}^{2} \cap B_{4 / \alpha}^{p}: i_{1}+i_{2} \in 2 \mathbb{Z}\right\}
$$

where $B_{r}^{p}=\left\{x \in \mathbb{R}^{2}:\|x\|_{p}<r\right\}$. Note that $\mathcal{N}_{\alpha}^{p}=\{0\}$ if $\alpha>4$ :

We consider only $\alpha$ with such a unique minimizer. The subset $\mathcal{N}_{\alpha}^{p}$ of $\mathbb{Z}^{2}$ will be called the nucleus of the process. Correspondingly, we have the continuum set $P_{\alpha}^{p}$ obtained as the convexification of $N_{\alpha}^{p}$. Note that $P_{\alpha}^{1}$ and $P_{\alpha}^{\infty}$ are always squares, but for the other $p$ the form of $P_{\alpha}^{p}$ does depend on $\alpha$.

The most delicate argument in the study of the discrete scheme is the characterization of the sets $A_{k}^{\alpha}$ for $k>1$. Similarly to the case $k=1$, this is done by covering $\mathbb{R}^{2}$ with a family of small sets, mainly squares and rectangles, in each of which we prove that the minimal set is again the even checkerboard. In order to construct this covering, we have to define the "edges" of the nucleus $\mathcal{N}_{\alpha}^{p}$ and consider separately the regions of $\mathbb{R}^{2}$ that project on those edges according to the $p$-distance. At this point, we have a technical hypothesis to add; namely, that all such regions are infinite (which is satisfied if these edges enclose a convex shape but may not be the case for some exceptional values of $\alpha$ ). The complex construction of this covering is the reason why we limit our analysis to a two-dimensional setting. 
With this characterization, using (1.5) we immediately have that the centers of the squares in $A_{k}^{\alpha}$ are exactly the points $i \in \mathbb{Z}^{2}$ with $i_{1}+i_{2} \in 2 \mathbb{Z}$ and distance not greater than $4 / \alpha$ from $A_{k-1}^{\alpha}$, so that

$$
A_{k}^{\alpha} \cap \mathbb{Z}^{2}=\left(A_{k-1}^{\alpha} \cap \mathbb{Z}^{2}\right)+\left(A_{1}^{\alpha} \cap \mathbb{Z}^{2}\right) .
$$

In a sense, every square in $A_{k-1}^{\alpha}$ acts as the "center" of a nucleus. Note in this step that if $A_{1}^{\alpha}$ were not unique, then we would have an "increasing non-uniqueness" of $A_{k}^{\alpha}$, which in particular may even not be the intersection of the square checkerboard with a convex region.

Since the centers of the squares in $A_{k}^{\alpha}$ are obtained as sums of $k$ elements in $\mathcal{N}_{\alpha}^{p}$, a result on Minkowski sums of sets shows then that the convex envelope of $A_{k}^{\alpha} \cap \mathbb{Z}^{2}$ is the convex envelope of $k \mathcal{N}_{\alpha}^{p}$, which is an interesting and not a trivial fact. At this point, we can go back to the original problem and describe the discrete orbits

$$
E_{k}^{\varepsilon, \tau}=\varepsilon A_{k}^{\alpha}=\varepsilon k P_{\alpha}^{p}, \quad E^{\varepsilon}(t)=E_{\lfloor\varepsilon t / \tau\rfloor}^{\varepsilon, \tau}=\varepsilon\left\lfloor\frac{\alpha}{\varepsilon}\right\rfloor P_{\alpha}^{p} .
$$

Letting $\varepsilon \rightarrow 0$, we then conclude that the desired evolution is a linear evolution of sets

$$
E(t)=\alpha t P_{\alpha}^{p}
$$

Note that $P_{\alpha}^{p}=\{0\}$ and hence the evolution is pinned if $\alpha>4$. Moreover, remarking that $\alpha P_{\alpha}^{p} \sim B_{4}^{p}$ for $\alpha$ small, we also recover the case $\alpha=0$, corresponding to the regime $\varepsilon^{2}<<\tau$, for which $E(t)=4 t B_{1}^{p}$.

We note that in Braides et al. (2010), the same discretization approach had been followed for the (positive) perimeter and non-trivial initial data. The resulting evolution therein is a discretized motion by square-crystalline curvature (see Almgren and Taylor 1995), which highlights the anisotropy of the lattice intervening in the perimeter part, while the effect of the dissipation is confined in the form of the mobility. In the present analysis, the effect of the dissipation and that of the perimeter parts is combined in the determination of the shape of the nucleus, but the perimeter term actually acts as an approximation of an area and is less relevant for small values of $\alpha$. Note that our discretization approach can be regarded as a "backward" version of Braides et al. (2010) if the index $k$ is considered as parameterizing negative time (see Braides 2013, Section 10.2). Other analyses of minimizing movements on lattices related to the perimeter can be found in Braides and Scilla (2013a), Scilla (2014, 2020) and Ruf (2018). We note that checkerboard, stripes and other structures arise in antiferromagnetic systems related to maximization of the perimeter (see Braides and Cicalese 2017 for a variational analysis in terms of $\Gamma$-convergence, and the wide literature in Statistical Mechanics, e.g., Giuliani et al. 2011; Daneri and Runa 2019). Some cases in which microstructures on lattices are involved and produce interesting variants of motion by crystalline curvature are studied in Braides et al. (2016) and Braides and Solci (2016). For an overview on geometric motion on planar lattices, see the recent lecture notes (Braides and Solci 2021). 
Even though our interest is mainly in the analytical issues of this nucleation process, it is suggestive and interesting to connect this work with the process of biomineralization, where nucleation occurs via the formation of a small nucleus of a new phase inside the large volume of the old phase (see, e.g., De Yoreo and Vekilov 2003). At very small size, adding even one more molecule increases the free energy of the system and this produces, on average, the dissolution of the nucleus. Above a threshold, when the contribution of the surface free energy becomes negligible, every addition of a molecule to the lattice lowers the free energy and allows for the growth of the nucleus. In this direction, lattice systems have been widely used as a simple model in simulations of complex phenomena, as the vapor-liquid nucleation (see, e.g., Kalikmanov 2013, Section 8.9). From a completely different point of view, our structure results can be related to the investigation of the influences of environmental heterogeneities on the spatial self-organization of microbial communities (see, e.g., Ciccarese 2020; Mimura et al. 2000); in particular, how interactions of different type (mutualism/commensalism) between competing neighboring genotypes and their mutual distance can produce spatial patterns of varying complexity and intermixing, as a random distribution, a spatial segregation or even a checkerboard, and how they may affect the collective behaviour and the rate of growth of the colony.

Outline of the paper In Sect. 2, we fix some notation and recall some preliminaries in discrete geometry. We introduce the class of admissible sets that we will consider throughout the paper, and the notions of effective boundary and discrete edge of a set. In Sect. 3, we define perimeter energies $P_{\varepsilon}$ and, for a general norm $\varphi$, dissipations $D_{\varepsilon}^{\varphi}$ we will deal with, together with the main functional $\mathcal{F}_{\varepsilon, \tau}^{\varphi}$. Correspondingly, we introduce the time-discrete minimization scheme for a suitably scaled version of the energies $\mathcal{F}_{\varepsilon, \tau}^{\varphi}$ (Sect. 3.1).

The convergence analysis of this scheme at the regime $\varepsilon<<\tau$ is carried out in Sect. 4. In Sect. 5, we address the problem of determining the solutions of scheme (1.4) at the critical regime $\varepsilon=\alpha \tau$, under a monotonicity constraint on the discrete trajectories. We introduce here also a first restriction on the dissipations $D_{\varepsilon}^{\varphi}$, by requiring that $\varphi$ be an absolute norm, i.e., $\varphi(\mathbf{x})=\varphi\left(\left|x_{1}\right|,\left|x_{2}\right|\right)$. The explicit characterization of the first step $A_{\alpha}^{1}$ of the discrete evolution, provided with Proposition 27, is based on a local analysis by means of the $2 \times 2$-square tilings introduced in Sect. 5.1 and the key submodularity-type norm-inequality (5.8). In order to prove that an analogous structure result can be obtained for each step $A_{\alpha}^{k}, k \geq 2$, i.e., for minimizers of the energy $\mathcal{F}_{\alpha}^{\varphi}\left(\cdot, A_{\alpha}^{k-1}\right)$, we will assume that $\varphi$ is a symmetric absolute normalized norm (see Sect. 5.2), complying with a technical assumption (H3), and that the competitors fulfill suitable geometric assumptions (see (5.13)). The proof of this stability result, given with Proposition 30, is the content of Sect. 5.5 and relies on a localization argument only reminiscent of that used in the proof of Proposition 27, as we are forced to define a new covering outside every discrete edge contained in the effective discrete boundary of the current step $A_{\alpha}^{k-1}$. In Sect. 5.6, with Theorem 38 we characterize the time-discrete flow $\left\{A_{\alpha}^{k}\right\}_{k \geq 0}$ as a geometric iterative process, based on properties of Minkowski sums.

In Sect. 6, we describe the resulting limit evolutions and we prove the existence of a pinning threshold (see Definition 40). We conclude our analysis by exhibiting, in 
Sect. 6.1, some examples where both the microscopic and the limit evolutions can be explicitly characterized. The closing Sect. 6.2 contains some conjectures on evolutions without the monotonicity constraint.

\section{Notation and Preliminaries}

The generic point of $\mathbb{R}^{2}$ will be denoted by $\mathbf{x}=\left(x_{1}, x_{2}\right)$, the Euclidean norm by $|\cdot|$ in any dimension. The space of subsets of $\mathbb{R}^{2}$ with finite perimeter endowed with the Hausdorff distance $d_{\mathcal{H}}$ is denoted by $\mathcal{X}$, and the one-dimensional Hausdorff measure by $\mathcal{H}^{1}$ (see, for instance, Ambrosio et al. 2000).

The function $\varphi: \mathbb{R}^{2} \rightarrow[0,+\infty)$ denotes any norm in the plane. We use the standard notation for the $\ell^{p}$-norm, for every $1 \leq p \leq \infty$; that is,

$$
\|\mathbf{x}\|_{p}=\left(\left|x_{1}\right|^{p}+\left|x_{2}\right|^{p}\right)^{\frac{1}{p}} \text { if } 1 \leq p<\infty, \quad\|\mathbf{x}\|_{\infty}=\max \left\{\left|x_{1}\right|,\left|x_{2}\right|\right\} \text { if } p=\infty
$$

for every $\mathbf{x} \in \mathbb{R}^{2}$. For every $r>0, B_{r}^{\varphi}(\mathbf{x})=\left\{\mathbf{y} \in \mathbb{R}^{2}: \varphi(\mathbf{x}-\mathbf{y})<r\right\}$ is the open ball of radius $r$ and center $\mathbf{x}$ corresponding to the norm $\varphi$, while $q_{r}(\mathbf{x})=\mathbf{x}+[-r / 2, r / 2]^{2}$ is the $r$-square of side length $r$ centered at $\mathbf{x}$; when $\mathbf{x}=(0,0)$, we will use the shorthand $B_{r}^{\varphi}$ and $q_{r}$ in place of $B_{r}^{\varphi}(\mathbf{x})$ and $q_{r}(\mathbf{x})$, respectively. For every $\mathbf{x} \in \mathbb{R}^{2}, E \subseteq \mathbb{R}^{2}$ we set $d^{\varphi}(\mathbf{x}, E)=\inf _{\mathbf{y} \in E} \varphi(\mathbf{x}-\mathbf{y})$. The segment connecting $\mathbf{x}_{1}, \mathbf{x}_{2} \in \mathbb{R}^{2}$ is denoted by $\left[\mathbf{x}_{1}, \mathbf{x}_{2}\right]:=\left\{\mathbf{y} \in \mathbb{R}^{2}: \mathbf{y}=s \mathbf{x}_{1}+(1-s) \mathbf{x}_{2}, s \in[0,1]\right\}$.

Definition 1 Given two unit vectors $\mathbf{v}_{1}, \mathbf{v}_{2} \in \mathbb{S}^{1}, \theta\left(\mathbf{v}_{2}, \mathbf{v}_{1}\right) \in[-\pi, \pi]$ denotes the signed angle between $\mathbf{v}_{1}$ and $\mathbf{v}_{2}$, defined as

$$
\theta\left(\mathbf{v}_{2}, \mathbf{v}_{1}\right)=\left(\theta_{2}-\theta_{1}+\pi(\bmod .2 \pi)\right)-\pi
$$

where $\theta_{1}$ and $\theta_{2}$ are the angles corresponding to the exponential representations of $\mathbf{v}_{1}$ and $\mathbf{v}_{2}$, respectively.

Let $\mathbb{Z}^{2}$ be the standard square lattice. We consider the partition of $\mathbb{Z}^{2}$ given by $\mathbb{Z}^{2}=\mathbb{Z}_{e}^{2} \cup \mathbb{Z}_{o}^{2}$, where $\mathbb{Z}_{e}^{2}=\left\{\mathbf{i} \in \mathbb{Z}^{2}: i_{1}+i_{2} \in 2 \mathbb{Z}\right\}$ and $\mathbb{Z}_{o}^{2}=(1,0)+\mathbb{Z}_{e}^{2}$.

We will call a lattice set any subset $\mathcal{J} \subseteq \mathbb{Z}^{2}$, and \#J denotes its cardinality. We also recall that the boundary of a lattice set $\mathcal{J}$ is the set

$$
\partial \mathcal{J}=\left\{\mathbf{i} \in \mathcal{J} \mid \text { there exists } \mathbf{j} \in \mathbb{Z}^{2} \backslash \mathcal{J}:|\mathbf{i}-\mathbf{j}|=1\right\}
$$

Given a lattice set $\mathcal{J}$, the convex hull of $\mathcal{J}$ is the smallest convex subset of $\mathbb{R}^{2}$ containing $\mathcal{J}$, which is denoted by conv $(\mathcal{J})$. A polygon whose vertices are points of the lattice is said a lattice polygon. The set conv $(\mathcal{J})$ is an example of a (convex) lattice polygon, for every $\mathcal{J} \subset \mathbb{Z}^{2}$.

Let $\varepsilon>0$ be a fixed parameter and consider the lattice $\varepsilon \mathbb{Z}^{2}$. All the notation given above for subsets of $\mathbb{Z}^{2}$ extends also to subsets of $\varepsilon \mathbb{Z}^{2}$. We identify any lattice set $\mathcal{J} \subset \varepsilon \mathbb{Z}^{2}$ with the subset $E(\mathcal{J})$ of $\mathbb{R}^{2}$ given by the union of $\varepsilon$-squares centered at points 
of J; namely,

$$
E(\mathcal{J}):=\bigcup_{\mathbf{i} \in \mathcal{J}} q_{\varepsilon}(\mathbf{i})
$$

Accordingly, we define the class of admissible sets as

$$
\mathcal{D}_{\varepsilon}:=\left\{E \subset \mathbb{R}^{2}: E=E(\mathcal{J}) \text { for some lattice set } \mathcal{J} \subseteq \varepsilon \mathbb{Z}^{2}\right\}
$$

and to each set $E \in \mathcal{D}_{\varepsilon}$ we associate the lattice set $Z_{\varepsilon}(E):=E \cap \varepsilon \mathbb{Z}^{2}$, the set of centers of $E$. When $\varepsilon=1$, we will simply write $\mathcal{D}$ and $Z(E)$ in place of $\mathcal{D}_{1}$ and $Z_{1}(E)$, respectively.

Definition 2 (The classes of checkerboard sets) We introduce the classes of even and odd $\varepsilon$-checkerboard sets

$$
\mathcal{A}_{\varepsilon}^{e}=\left\{E \in \mathcal{D}_{\varepsilon}: Z_{\varepsilon}(E) \subseteq \varepsilon \mathbb{Z}_{e}^{2}\right\}
$$

and analogously the class $\mathcal{A}_{\varepsilon}^{o}$ by requiring that $\mathcal{J} \subseteq \varepsilon \mathbb{Z}_{o}^{2}$. We refer to $E\left(\varepsilon \mathbb{Z}_{e}^{2}\right)$ and $E\left(\varepsilon \mathbb{Z}_{o}^{2}\right)$ as the even and odd $\varepsilon$-checkerboard, respectively. In the following, we will write $\mathcal{D}, \mathcal{A}^{e}, \mathcal{A}^{o}$ in place of $\mathcal{D}_{1}, \mathcal{A}_{1}^{e}, \mathcal{A}_{1}^{o}$, and we will use the shorthand checkerboard set (in place of "1-checkerboard set") to denote any set in $\mathcal{A}^{e}$ and $\mathcal{A}^{o}$.

\subsection{Preliminaries on Lattice Geometry}

For our purposes, we fix some notation and introduce some basic definitions in lattice geometry that will be useful for the analysis performed in Sect. 5.4.

Definition 3 A lattice set $\mathcal{J} \subseteq \mathbb{Z}_{e}^{2}$ is said to be $\mathbb{Z}_{e}^{2}$-convex if $\operatorname{conv}(\mathcal{J}) \cap \mathbb{Z}_{e}^{2}=\mathcal{J}$. Analogously, $\mathcal{J} \subseteq \mathbb{Z}_{o}^{2}$ is $\mathbb{Z}_{o}^{2}$-convex if $\operatorname{conv}(\mathcal{J}) \cap \mathbb{Z}_{o}^{2}=\mathcal{J}$. Accordingly, we define the subclass $\mathcal{A}_{\mathrm{conv}}^{e} \subset \mathcal{D}$ as

$$
\mathcal{A}_{\text {conv }}^{e}=\left\{E \in \mathcal{D}: Z(E) \text { is } \mathbb{Z}_{e}^{2} \text {-convex }\right\}
$$

and, analogously, the subclass $\mathcal{A}_{\text {conv }}^{o}$ by requiring $Z(E)$ to be $\mathbb{Z}_{o}^{2}$-convex. We also set the class $\mathcal{A}_{\text {conv }}:=\mathcal{A}_{\text {conv }}^{e} \cup \mathcal{A}_{\text {conv }}^{o}$.

The notion of convex lattice set has already been given for $\mathcal{J} \subset \mathbb{Z}^{2}$ (see, for instance, Gardner et al. 2005). Note that $\mathcal{J}$ is $\mathbb{Z}_{e}^{2}$-convex if and only if there exists a convex set $K \subset \mathbb{R}^{2}$ such that $\mathcal{J}=K \cap \mathbb{Z}_{e}^{2}$, and the same holds for $\mathbb{Z}_{o}^{2}$-convex sets.

For every lattice set $\mathcal{J} \subseteq \mathbb{Z}_{e}^{2}$ (or $\mathbb{Z}_{o}^{2}$ ), there holds $\partial \mathcal{J}=\mathcal{J}$, since $\mathcal{J}$ consists of isolated points of $\mathbb{Z}^{2}$. Since in the following we will deal with checkerboard sets, we need a finer definition of boundary for such lattice sets. 

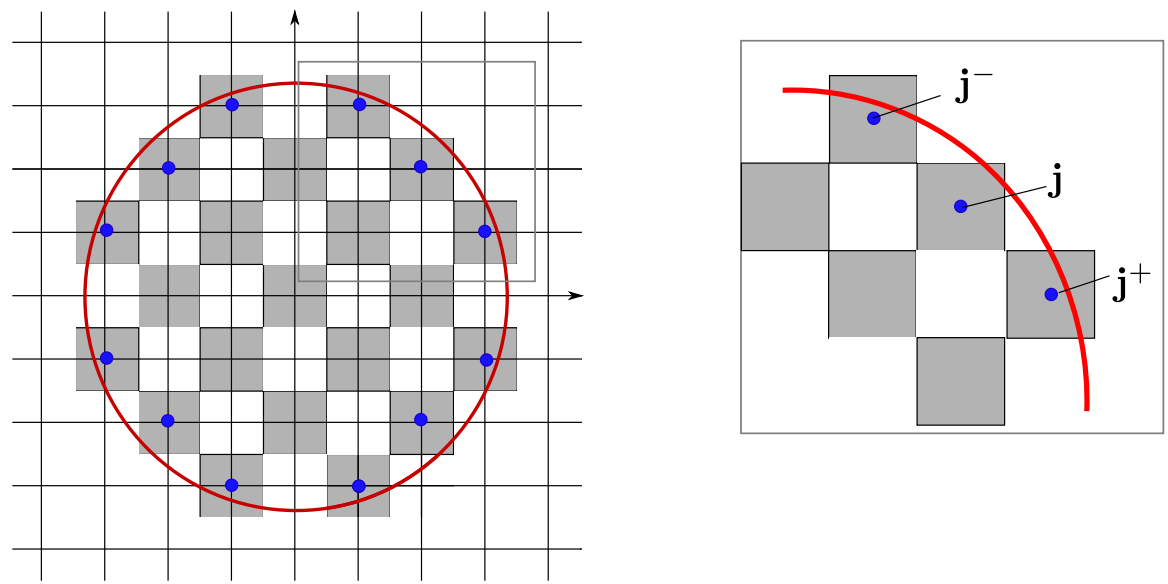

Fig. 1 The (discrete) effective boundary of $E$ (in blue). (Color figure online)

○

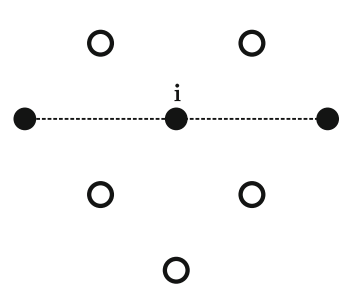

0

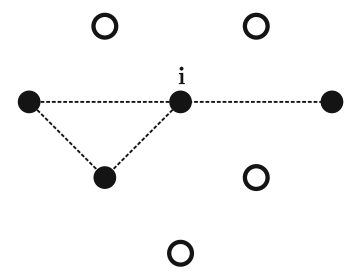

0

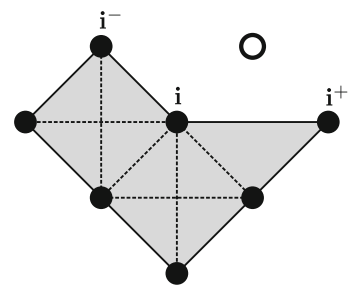

Fig. 2 The black dots are lattice points of J. The first two figures are different examples of "degenerate" i. On the right an example of a non-degenerate $\mathbf{i}$ and corresponding $\mathbf{i}^{-}$and $\mathbf{i}^{+}$; in gray polygon $\mathcal{P}$

Definition 4 Let $\mathcal{J} \subset \mathbb{Z}_{e}^{2}$ be a lattice set. We define the effective (discrete) boundary of $\mathcal{J}$ as

$$
\partial^{\text {eff } \mathcal{J}}=\left\{\mathbf{j} \in \mathcal{J}: \text { there exists } \mathbf{j}_{0} \in \mathbb{Z}_{e}^{2} \backslash \mathcal{J} \text { such that }\left|\mathbf{j}-\mathbf{j}_{0}\right|=\sqrt{2}\right\} .
$$

The same definition is given for lattice sets $\mathcal{J} \subset \mathbb{Z}_{o}^{2}$. Let $E \in \mathcal{A}^{e} \cup \mathcal{A}^{o}$, we will write $\partial^{\text {eff }} E=\partial^{\text {eff }} Z(E)$, see Fig. 1 .

Given $E \in \mathcal{A}^{e} \cup \mathcal{A}^{o}$, consider $\mathbf{i} \in \partial^{\text {eff }} E$. We set $\mathcal{J}=\left\{\mathbf{j} \in Z(E):\|\mathbf{j}-\mathbf{i}\|_{1} \leq 2\right\}$. Then, $\mathbf{i}$ is said to be non-degenerate if the set

$$
\bigcup_{\substack{\mathbf{j}_{1}, \mathbf{j}_{2} \in \mathcal{J} \\\left|\mathbf{j}_{1}-\mathbf{j}_{2}\right| \leq 2}}\left[\mathbf{j}_{1}, \mathbf{j}_{2}\right]
$$

is the boundary of a triangulation of a simple polygon $\mathcal{P}$. Then, we can define two boundary points $\mathbf{i}^{-}, \mathbf{i}^{+} \in \partial^{\text {eff }} E$ as the vertices of $\mathcal{P}$, respectively, preceding and following $\mathbf{i}$ in the clockwise orientation of $\partial \mathcal{P}$, as depicted in Fig. 2. We will say that $\mathbf{i}^{-}$precedes $\mathbf{i}$ and that $\mathbf{i}^{+}$follows $\mathbf{i}$. 


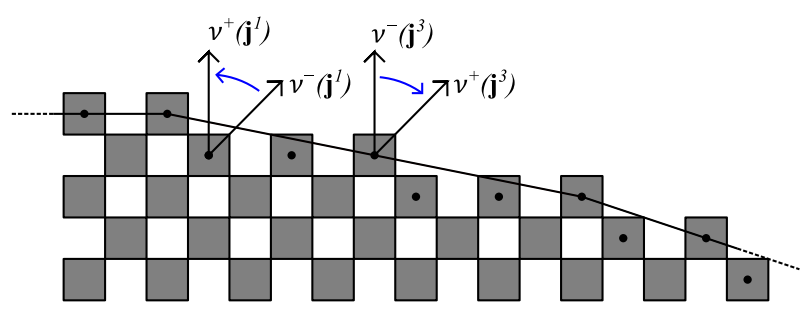

Fig. 3 A discrete vertex of $E$ may be a boundary point (not a vertex) of $\operatorname{conv}(Z(E)$ )

In the sequel, we will often consider the following non-degeneracy condition on sets $E \in \mathcal{A}_{\text {conv }}$;

$$
\text { every } \mathbf{i} \in \partial^{\text {eff }} E \text { is non-degenerate. }
$$

Condition (2.3) allows to define an orientation of $\partial^{\text {eff }} E$, since for every $\mathbf{i} \in \partial^{\text {eff }} E$ we can define $\mathbf{i}^{-}$and $\mathbf{i}^{+}$as above. The following definitions are therefore well-posed.

Definition 5 (Discrete convex vertices) Let $E \in \mathcal{A}_{\text {conv }}$ satisfy (2.3). Given $\mathbf{j} \in \partial^{\text {eff }} E$, let $\mathbf{j}^{+}$(resp., $\mathbf{j}^{-}$) follow (resp., precede) $\mathbf{j}$ in $\partial^{\text {eff }} E$ in the clockwise orientation. We define the right and left outward unit normal vector at $\mathbf{j}$ as

$$
\boldsymbol{v}^{+}(\mathbf{j}):=\frac{\left(j_{2}-j_{2}^{+}, j_{1}^{+}-j_{1}\right)}{\sqrt{\left(j_{2}^{+}-j_{2}\right)^{2}+\left(j_{1}^{+}-j_{1}\right)^{2}}}, \quad \boldsymbol{v}^{-}(\mathbf{j}):=\frac{\left(j_{2}^{-}-j_{2}, j_{1}-j_{1}^{-}\right)}{\sqrt{\left(j_{2}-j_{2}^{-}\right)^{2}+\left(j_{1}-j_{1}^{-}\right)^{2}}},
$$

respectively. Then, we say that $\mathbf{j}$ is a discrete convex vertex (or discrete vertex) if

$$
\theta\left(\boldsymbol{v}^{+}(\mathbf{j}), \boldsymbol{v}^{-}(\mathbf{j})\right)<0
$$

where $\theta$ is introduced in Definition 1.

Remark 6 (Vertices and discrete vertices) The definition of discrete vertex given above is motivated by the fact that the vertices of $\operatorname{conv}(Z(E))$ are discrete (convex) vertices of $E$, whereas points $\mathbf{j} \in \partial^{\text {eff }} E$ such that

$$
\theta\left(\boldsymbol{v}^{+}(\mathbf{j}), \boldsymbol{v}^{-}(\mathbf{j})\right)>0
$$

are always contained in the interior of $\operatorname{conv}(Z(E))$ (Fig. 3). This choice will also facilitate the definition of discrete edge (see Definition 7 ).

Note that we may have discrete vertices of $E$ lying on the boundary of $\operatorname{conv}(Z(E))$ which are not vertices of $\operatorname{conv}(Z(E))$ (see Fig. 3), and discrete vertices of $E$ in the interior of $\operatorname{conv}(Z(E))$, as well (see Fig. 4). 


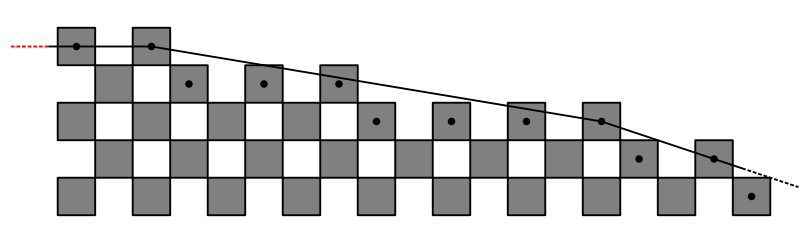

Fig. 4 An example of a discrete vertex of $E$ contained in the interior of $\operatorname{conv}(Z(E))$

(a)

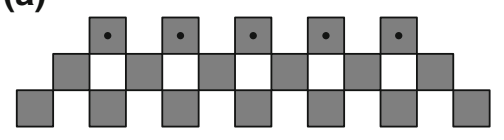

(c)

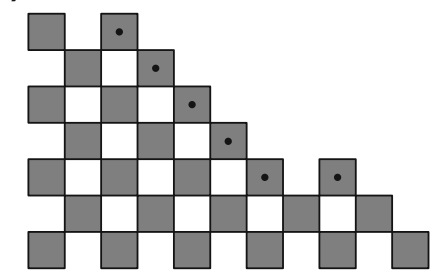

(b)

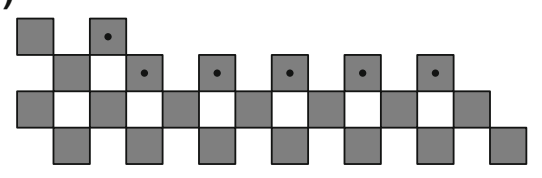

(d)

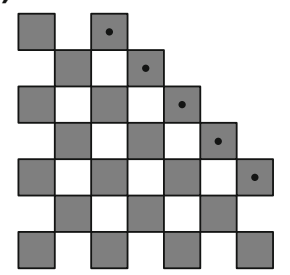

Fig. 5 Some examples of discrete edges

Definition 7 (Discrete edges) Let $E \in \mathcal{A}_{\text {conv }}$ satisfy (2.3). We define a discrete edge as a set of consecutive points of $\partial^{\text {eff }} E$, say $\ell=\left\{\mathbf{j}^{l}\right\}_{l=0}^{L}$ where $L \geq 2$ and $\mathbf{j}^{0}$ and $\mathbf{j}^{L}$ are discrete vertices. We define the outward unit normal vector of the discrete edge $\ell$ as

$$
\boldsymbol{v}(\ell):=\frac{\left(j_{2}^{0}-j_{2}^{L}, j_{1}^{L}-j_{1}^{0}\right)}{\sqrt{\left(j_{2}^{L}-j_{2}^{0}\right)^{2}+\left(j_{1}^{L}-j_{1}^{0}\right)^{2}}} .
$$

We denote by $\mathcal{E}(E)$ the set of all discrete edges $\ell \subset \partial^{\text {eff }} E$.

Let $E \in \mathcal{A}_{\text {conv }}$ satisfy (2.3). For every $\ell \in \partial^{\text {eff }} E$, we define the slope of $\ell$ as

$$
s(\ell):=\frac{v(\ell)_{1}}{v(\ell)_{2}} \in[-\infty,+\infty],
$$

where $v(\ell)_{k}, k=1,2$ indicate the components of $\boldsymbol{v}(\ell)$, with the convention that $\frac{ \pm 1}{0}= \pm \infty$.

Remark 8 We list all the possible cases of discrete edges of sets $E \in \mathcal{A}_{\text {conv }}$ satisfying (2.3) that are symmetric with respect to the axes and the bisectors $x_{2}= \pm x_{1}$. Such symmetric sets will play a central role in the sequel of the paper. Up to rotations of angle $k \pi$ and reflections, we can restrict this characterization to discrete edges $\ell \in \mathcal{E}(E)$ such that $\ell=\left\{\mathbf{j}^{l}\right\}_{l=0}^{L} \subset\left\{\mathbf{x} \in \mathbb{R}^{2}: x_{2}>0\right\}$ having $s(\ell) \in[0,1]$. We have the following characterization: 
(i) if $s(\ell)=0$, then $\mathbf{j}^{l}=\mathbf{j}^{l-1}+(2,0)$ for every $1 \leq l \leq L$;

(ii) if $s(\ell) \in\left(0, \frac{1}{3}\right]$, then $\mathbf{j}^{l}=\mathbf{j}^{l-1}+(2,0)$ for every $1<l \leq L$ and $\mathbf{j}^{1}=\mathbf{j}^{0}+(1,-1)$;

(iii) if $s(\ell) \in\left(\frac{1}{3}, 1\right)$, then $\mathbf{j}^{l}=\mathbf{j}^{l-1}+(1,-1)$ for every $1 \leq l<L$ and $\mathbf{j}^{L}=$ $\mathbf{j}^{L-1}+(2,0)$

(iv) if $s(\ell)=1$, then $\mathbf{j}^{l}=\mathbf{j}^{l-1}+(1,-1)$ for every $1 \leq l \leq L$.

These four types of discrete edge are pictured in Fig. 5a-d, respectively.

Definition 9 For every norm $\varphi$ and every $E \in \mathcal{D}$, we introduce the projection map of integer points on $E$; that is, the set-valued map $\pi_{E}^{\varphi}: \mathbb{Z}^{2} \rightarrow \mathcal{P}\left(\mathbb{Z}^{2}\right)$ defined as

$$
\pi_{E}^{\varphi}(\mathbf{j}):=\underset{\mathbf{j}^{\prime} \in Z(E)}{\operatorname{argmin}} \varphi\left(\mathbf{j}-\mathbf{j}^{\prime}\right)
$$

\subsection{Minkowski Sum of Sets}

We recall that the Minkowski sum of sets $A$ and $B$ is defined as $A+B=\{a+b \mid a \in$ $A, b \in B\}$, and $A+\emptyset=\emptyset$. If $m \in \mathbb{N}$, we denote by $m A$ the set $\{m a \mid a \in A\}$, and if $A$ is non-empty, we will often write $A[m]$ to indicate the sum $A+A+\cdots+A$ $m$-times. Among the many properties of Minkowski sum, we recall the commutability of Minkowski sum and the compatibility to the operation of taking the convex hull; that is,

$$
\operatorname{conv}(A+B)=\operatorname{conv}(A)+\operatorname{conv}(B) .
$$

We recall without proof a result about the Minkowski sum of two convex polygons (see, e.g., Barki et al. 2009).

Proposition 10 Let $A$ and $B$ be convex polygons in $\mathbb{R}^{2}$. Let $L_{A}:=\left\{l_{i, A}\right\}_{i=1, \ldots, n}$ and $L_{B}:=\left\{l_{j, B}\right\}_{j=1, \ldots, m}$ be the sets of the edges of $A$ and $B$, respectively. Let $V_{A}:=\left\{v_{i, A}\right\}_{i=1, \ldots, n}$ and $\mathcal{V}_{B}:=\left\{v_{j, B}\right\}_{j=1, \ldots, m}$ be the sets of the outer normal vectors of $A$ and $B$, respectively. Then,

(i) if $\mathcal{V}_{A} \cap \mathcal{V}_{B}=\emptyset$, then $L_{A+B}=L_{A} \cup L_{B}$ and $\mathcal{V}_{A+B}=\mathcal{V}_{A} \cup \mathcal{V}_{B}$;

(ii) if $\left|\mathcal{V}_{A} \cap \mathcal{V}_{B}\right|=p, 1 \leq p \leq \min \{n, m\}$, then $\left|L_{A+B}\right|=n+m-p$. More precisely, if $v_{i, A}=v_{j, B}$ for some $i \in\{1, \ldots, n\}$ and $j \in\{1, \ldots, m\}$, then $l_{i, A}+l_{j, B} \in L_{A+B}$, $l_{i, A} \notin L_{A+B}, l_{j, B} \notin L_{A+B}$ and $v_{i, A}=v_{j, B} \in V_{A+B}$. If, instead, $v_{i, A} \neq v_{j, B}$, then $l_{i, A} \in L_{A+B}, l_{j, B} \in L_{A+B}, v_{i, A} \in \mathcal{V}_{A+B}$ and $v_{j, B} \in \mathcal{V}_{A+B}$.

In particular, if $A=B$, then $L_{A+A}=\left\{l_{i, A}+l_{i, A}\right\}_{i=1, \ldots, n}$ and $\mathcal{V}_{A+A}=\mathcal{V}_{A}$.

\subsection{The Lattice Point-Counting Problem: $m$-Fold Minkowski Sums}

Let $B=\left\{\mathbf{w}_{1}, \mathbf{w}_{2}\right\}$ be a basis of $\mathbb{R}^{2}$. The set

$$
\Lambda=\Lambda(B):=\left\{z_{1} \mathbf{w}_{1}+z_{2} \mathbf{w}_{2}: z_{1}, z_{2} \in \mathbb{Z}\right\}
$$


is called a lattice of $\mathbb{R}^{2}$ with basis $B$. The corresponding fundamental cell is defined as

$$
\left\{\mu_{1} \mathbf{w}_{1}+\mu_{2} \mathbf{w}_{2}: \mu_{1}, \mu_{2} \in[0,1)\right\}
$$

whose area is $|\operatorname{det}(B)|$. It can be checked that the area of the fundamental cell is independent of the choice of the basis and is referred to as the determinant of $\Lambda$, $\operatorname{det}(\Lambda)$. Lattices are additive subgroups of $\mathbb{R}^{2}$ and they are discrete sets. Examples of lattices are the standard lattice $\mathbb{Z}^{2}$, with basis $\{(1,0),(0,1)\}$ and $\left|\operatorname{det}\left(\mathbb{Z}^{2}\right)\right|=1$, and the "checkerboard lattice" $\mathbb{Z}_{e}^{2}$, with basis $\{(-1,1),(1,1)\}$ and $\left|\operatorname{det}\left(\mathbb{Z}_{e}^{2}\right)\right|=2 . \mathbb{Z}_{o}^{2}$ is not a lattice, since $(1,0)+(0,1)=(1,1) \notin \mathbb{Z}_{0}^{2}$.

It will be useful in the sequel to obtain an estimate on the number of the lattice points contained in $m \mathcal{Q}, m \in \mathbb{N}$ for $\mathcal{Q}$ lattice convex polygon. For this, we first recall a fundamental result for counting the lattice points in $Q$.

Theorem 11 (Pick's Theorem, Pick 1899) Let $\Lambda$ be any lattice in $\mathbb{R}^{2}$, let $\mathcal{J} \subset \Lambda$ be a finite set and $Q=\operatorname{conv}(\mathcal{J})$. Then,

$$
\#(\mathcal{Q} \cap \Lambda)=\frac{1}{|\operatorname{det}(\Lambda)|}|\mathcal{Q}|+\frac{1}{2} \#(\partial \mathcal{Q} \cap \Lambda)+1,
$$

where $|Q|$ is the area of $\mathcal{Q}$ and $\partial \mathcal{Q}$ its topological boundary.

A non-trivial problem in discrete geometry is the comparison between the set of the lattice points contained in the homothetic copy $m \mathcal{Q}$ of a convex lattice polyhedron $\mathcal{Q}$ with the $m$-fold Minkowski sum $\left(\mathcal{Q} \cap \mathbb{Z}^{n}\right)[m], n \geq 2$ (see, e.g., Lindner and Roch 2011). It will be sufficient for our purposes here to mention that in the two-dimensional setting the two lattice sets coincide (see Lindner and Roch 2011, Corollary 2.4). Moreover, an inspection of the proof reveals that the result still holds if we replace $\mathbb{Z}^{2}$ with any two-dimensional lattice $\Lambda$.

Proposition 12 Let $\Lambda$ be any lattice in $\mathbb{R}^{2}$, let $\mathcal{J} \subset \Lambda$ be a finite set and $Q=\operatorname{conv}(\mathcal{J})$ be two-dimensional. Then, the equality

$$
(\mathcal{Q} \cap \Lambda)[m]=(m \mathcal{Q}) \cap \Lambda
$$

holds for every $m \in \mathbb{N}$.

Now, in view of Proposition 12 and by iterating formula (2.7), Pick's theorem generalizes to $m \mathcal{Q}, m \geq 1$, as

$$
\#((m \mathcal{Q}) \cap \Lambda)=\frac{1}{|\operatorname{det}(\Lambda)|}|\mathcal{Q}| m^{2}+\frac{1}{2} \#(\partial \mathcal{Q} \cap \Lambda) m+1 .
$$

\subsection{Submodularity and Absolute Norms}

We briefly recall the concept of submodularity which is well known in discrete convex analysis (see, e.g., Murota 2003, Ch. 2, eq. (2.17)). Setting $\mathbb{R}_{+}^{2}:=\left\{\mathbf{x}=\left(x_{1}, x_{2}\right) \in\right.$ 
$\left.\mathbb{R}^{2} \mid x_{1}, x_{2} \geq 0\right\}$, for every $\mathbf{x}, \mathbf{y} \in \mathbb{R}^{2}$ we define

$$
\mathbf{x} \vee \mathbf{y}:=\left(\max \left\{x_{1}, y_{1}\right\}, \max \left\{x_{2}, y_{2}\right\}\right) \text { and } \mathbf{x} \wedge \mathbf{y}:=\left(\min \left\{x_{1}, y_{1}\right\}, \min \left\{x_{2}, y_{2}\right\}\right)
$$

A function $f: \mathbb{R}_{+}^{2} \rightarrow \mathbb{R}$ is said to be submodular if it satisfies the following inequality

$$
f(\mathbf{x} \vee \mathbf{y})+f(\mathbf{x} \wedge \mathbf{y}) \leq f(\mathbf{x})+f(\mathbf{y}), \text { for every } \mathbf{x}, \mathbf{y} \in \mathbb{R}_{+}^{2} .
$$

It is known (see Marinacci and Montrucchio 2008, Proposition 5) that every positively homogeneous function defined in the cone $\mathbb{R}_{+}^{2}$ is subadditive if and only if it is submodular. In particular, this yields that every absolute norm $\varphi$ (i.e., $\varphi(\mathbf{x})$ depends only on $\left|x_{1}\right|$ and $\left|x_{2}\right|$ ) complies with (2.10). We recall that an absolute norm is monotonic:

$$
\left|x_{1}\right| \leq\left|y_{1}\right| \text { and }\left|x_{2}\right| \leq\left|y_{2}\right| \text { imply } \varphi(\mathbf{x}) \leq \varphi(\mathbf{y})
$$

\section{Setting of the Problem}

We will deal with negative discrete perimeters; that is, the Euclidean perimeter functional (with negative sign) restricted to $\mathcal{D}_{\varepsilon}$ relaxed to the space $X$. Namely, we define the functionals $F_{\varepsilon}: X \rightarrow(-\infty,+\infty]$ as

$$
F_{\varepsilon}(E)= \begin{cases}-\mathcal{H}^{1}(\partial E) & E \in \mathcal{D}_{\varepsilon} \\ +\infty & \text { otherwise }\end{cases}
$$

Note that these energies are related to the corresponding interaction energies defined on lattice sets

$$
F_{\varepsilon}^{\text {lat }}(\mathcal{J})=-\varepsilon \#\left\{(\mathbf{i}, \mathbf{j}) \in \varepsilon \mathbb{Z}^{2} \times \varepsilon \mathbb{Z}^{2}|\mathbf{i} \in \mathcal{J}, \mathbf{j} \notin \mathcal{J},| \mathbf{i}-\mathbf{j} \mid=\varepsilon\right\}
$$

where $\mathcal{J} \subset \varepsilon \mathbb{Z}^{2}$, and $F_{\varepsilon}^{\text {lat }}\left(Z_{\varepsilon}(E)\right)=F_{\varepsilon}(E)$. The functionals $F_{\varepsilon}$, in turn, may be seen as nearest-neighbor (NN) antiferromagnetic interaction energies associated to a lattice spin system; i.e., given $u: \varepsilon \mathbb{Z}^{2} \rightarrow\{-1,1\}$, one defines

$$
E_{\varepsilon}(u)=-\frac{\varepsilon}{4} \sum_{\substack{\mathbf{i}, \mathbf{j} \in \varepsilon \mathbb{Z}^{2} \\|\mathbf{i}-\mathbf{j}|=\varepsilon}}(u(\mathbf{i})-u(\mathbf{j}))^{2},
$$

whence $F_{\varepsilon}(E(\{u=1\}))=E_{\varepsilon}(u)$. The asymptotic behavior as $\varepsilon \rightarrow 0$ of energies like $F_{\varepsilon}$ has been studied, e.g., in Alicandro et al. (2006).

Let $\varphi: \mathbb{R}^{2} \rightarrow[0,+\infty)$ be a norm. For every pair of lattice sets $E, E^{\prime} \in \mathcal{D}_{\varepsilon}$, we define the dissipations

$$
D_{\varepsilon}^{\varphi}\left(E, E^{\prime}\right)=\varepsilon^{2} \sum_{\mathbf{i} \in Z_{\varepsilon}(E) \triangle Z_{\varepsilon}\left(E^{\prime}\right)} d_{\varepsilon}^{\varphi}\left(\mathbf{i}, \partial Z_{\varepsilon}\left(E^{\prime}\right)\right),
$$


where, given $\mathcal{J} \subset \varepsilon \mathbb{Z}^{2}, d_{\varepsilon}^{\varphi}$ denotes the discrete distance of any lattice point $\mathbf{i} \in \varepsilon \mathbb{Z}^{2}$ to $\partial \mathcal{J}$ defined as

$$
d_{\varepsilon}^{\varphi}(\mathbf{i}, \partial \mathcal{J})= \begin{cases}\inf \{\varphi(\mathbf{i}-\mathbf{j}) \mid \mathbf{j} \in \mathcal{J}\} & \text { if } \mathbf{i} \notin \mathcal{J} \\ \inf \left\{\varphi(\mathbf{i}-\mathbf{j}) \mid \mathbf{j} \in \varepsilon \mathbb{Z}^{2} \backslash \mathcal{J}\right\} & \text { if } \mathbf{i} \in \mathcal{J}\end{cases}
$$

Remark 13 In the sequel, the following integral formulation of the dissipation (3.2) will be useful. Indeed, for every $E^{\prime} \in X$ we set $d_{\varepsilon}^{\varphi}\left(\mathbf{i}, \partial E^{\prime}\right)=d_{\varepsilon}^{\varphi}\left(\mathbf{i}, \partial\left(E^{\prime} \cap \varepsilon \mathbb{Z}^{2}\right)\right)$. Furthermore, we can extend $d_{\varepsilon}^{\varphi}\left(\cdot, \partial E^{\prime}\right)$ to $\mathbb{R}^{2}$ by setting $d_{\varepsilon}^{\varphi}\left(\mathbf{x}, \partial E^{\prime}\right):=d_{\varepsilon}^{\varphi}\left(\mathbf{i}, \partial E^{\prime}\right)$ for $\mathbf{x} \in q_{\varepsilon}(\mathbf{i})$. Thus, for every $E, E^{\prime} \in \mathcal{X}$, let $E_{\varepsilon}, E_{\varepsilon}^{\prime} \in \mathcal{D}_{\varepsilon}$ be the corresponding discretizations; i.e., $Z_{\varepsilon}\left(E_{\varepsilon}\right)=E \cap \varepsilon \mathbb{Z}^{2}$ and the same for $E_{\varepsilon}^{\prime}$, we may write

$$
\int_{E \triangle E^{\prime}} d_{\varepsilon}^{\varphi}\left(\mathbf{x}, \partial E^{\prime}\right) \mathrm{d} \mathbf{x}=\varepsilon^{2} \sum_{\mathbf{i} \in Z_{\varepsilon}\left(E_{\varepsilon}\right) \triangle Z_{\varepsilon}\left(E_{\varepsilon}^{\prime}\right)} d_{\varepsilon}^{\varphi}\left(\mathbf{i}, \partial E_{\varepsilon}^{\prime}\right)=D_{\varepsilon}^{\varphi}\left(E_{\varepsilon}, E_{\varepsilon}^{\prime}\right)
$$

We will consider the dissipation in (3.2) as defined on every pair of sets of finite perimeter; i.e., $D_{\varepsilon}^{\varphi}: X \times X \rightarrow[0+\infty]$.

\subsection{The Time-Discrete Minimization Scheme with a Monotonicity Constraint}

For any $\varepsilon>0$ and $\tau>0$, let $F_{\varepsilon}$ and $D_{\varepsilon}^{\varphi}$ be defined as in (3.1) and (3.2), respectively. We introduce a discrete motion with underlying time step $\tau$ obtained by successive minimization. At each time step, we will minimize an energy $\mathcal{F}_{\varepsilon, \tau}^{\varphi}: \mathcal{X} \times \mathcal{X} \rightarrow$ $(-\infty,+\infty]$ defined as

$$
\mathcal{F}_{\varepsilon, \tau}^{\varphi}(E, F)=\varepsilon F_{\varepsilon}(E)+\frac{1}{\tau} D_{\varepsilon}^{\varphi}(E, F)
$$

with a monotonicity constraint on the discrete trajectories. Namely, we recursively define an increasing (with respect to inclusion) sequence $E_{\varepsilon, \tau}^{k}$ in $\mathcal{D}_{\varepsilon}$ by requiring the following:

$$
\left\{\begin{array}{l}
E_{\varepsilon, \tau}^{0}=q_{\varepsilon}, \\
E_{\varepsilon, \tau}^{k+1} \in \underset{E \in \mathcal{D}_{\varepsilon}, E \supset E_{\varepsilon, \tau}^{k}}{\operatorname{argmin}} \mathcal{F}_{\varepsilon, \tau}^{\varphi}\left(E, E_{\varepsilon, \tau}^{k}\right), \quad k \geq 0
\end{array}\right.
$$

In some cases, we will also analyze solutions of the corresponding unconstrained scheme; that is,

$$
\left\{\begin{array}{l}
E_{\varepsilon, \tau}^{0}=q_{\varepsilon} \\
E_{\varepsilon, \tau}^{k+1} \in \underset{E \in \mathcal{D}_{\varepsilon}}{\operatorname{argmin}} \mathcal{F}_{\varepsilon, \tau}^{\varphi}\left(E, E_{\varepsilon, \tau}^{k}\right), \quad k \geq 0
\end{array}\right.
$$


in which the minimization problems are performed over the whole class $\mathcal{D}_{\varepsilon}$. The discrete orbits associated with functionals $\mathcal{F}_{\varepsilon, \tau}^{\varphi}$ are thus defined by

$$
E_{\varepsilon, \tau}(t):=E_{\varepsilon, \tau}^{\lfloor t / \tau\rfloor}, \quad t>0
$$

We say that a curve $E:[0,+\infty) \rightarrow X$ is a minimizing movement for the problem (3.4) or (3.5) at regime $\tau-\varepsilon$ if it is pointwise limit (in the Hausdorff topology) of discrete orbits $E_{\varepsilon, \tau}$, as $\varepsilon, \tau \rightarrow 0$ up to subsequences.

Remark 14 (choice of scaling) The scale $\varepsilon$ in the energies $\varepsilon F_{\varepsilon}$ above is suggested by energetic considerations (see Braides and Scilla 2013b, (6)-(7)) and leads to a non-trivial limit of the discrete solutions defined in (3.6). This choice is motivated by the fact that $\varepsilon F_{\varepsilon}$ has a non-trivial $\Gamma$-limit, as we will show in Sect. 4.1. The energy scaling may also be seen as a time scaling of the discrete flow generated by taking the relaxation on $\mathcal{D}_{\varepsilon}$ of the energy functional $-\mathcal{H}^{1}$ (see Braides 2013, Section 10.2).

\section{Fast Convergences and the Emergence of a Critical Regime}

As remarked in (Braides 2013, Ch. 8), minimizing movements along families of functionals will depend in general on the regime $\tau-\varepsilon$; in our case, on the ratio between the two parameters $\tau$ and $\varepsilon$ that characterizes the motion. We first provide the following result that ensures a compactness property of the minimizers of the energies $\mathcal{F}_{\varepsilon, \tau}^{\varphi}$. In this section, $\varphi$ denotes a general norm, without any restriction.

Lemma 15 Let $F_{\varepsilon}$ and $D_{\varepsilon}^{\varphi}$ be defined as in (3.1) and (3.2), respectively, and $\mathcal{F}_{\varepsilon, \tau}^{\varphi}$ be as in (3.3). Let $E^{\prime} \in \mathcal{D}_{\varepsilon}$ be an admissible set. For every fixed $\tau>0$, consider

$$
E_{\varepsilon, \tau} \in \underset{E \in X}{\operatorname{argmin}} \mathcal{F}_{\varepsilon, \tau}^{\varphi}\left(E, E^{\prime}\right)
$$

Then, $Z_{\varepsilon}\left(E_{\varepsilon, \tau}\right) \subset E^{\prime}+B_{4 \tau}^{\varphi}$ and $d_{\mathcal{H}}\left(E_{\varepsilon, \tau}, E^{\prime}+B_{4 \tau}^{\varphi}\right)<3 \sqrt{2} \varepsilon$ for $\varepsilon$ small enough.

Proof For any $E \in \mathcal{D}_{\varepsilon}$, the variation of the energy $\mathcal{F}_{\varepsilon, \tau}^{\varphi}$ when removing a square of center $\mathbf{i} \in \varepsilon \mathbb{Z}^{2}$ is

$$
\mathcal{F}_{\varepsilon, \tau}^{\varphi}\left(E, E^{\prime}\right)-\mathcal{F}_{\varepsilon, \tau}^{\varphi}\left(E \backslash q_{\varepsilon}(\mathbf{i}), E^{\prime}\right) \leq 4 \varepsilon^{2}-\frac{\varepsilon^{2}}{\tau} d_{\varepsilon}^{\varphi}\left(\mathbf{i}, \partial E^{\prime}\right)
$$

which is strictly negative when $d_{\varepsilon}^{\varphi}\left(\mathbf{i}, \partial E^{\prime}\right)>4 \tau$, thus implying that $Z_{\varepsilon}\left(E_{\varepsilon, \tau}\right) \subset$ $E^{\prime}+B_{4 \tau}^{\varphi}$. Furthermore, since it is always convenient to add an isolated square $q_{\varepsilon}(\mathbf{j})$, if $\mathbf{j} \in Z_{\varepsilon}\left(E^{\prime}+B_{4 \tau}^{\varphi}\right)$, then for every $\mathbf{j} \in 3 \varepsilon \mathbb{Z}^{2} \cap E^{\prime}+B_{4 \tau}^{\varphi}$ we must have $E_{\varepsilon, \tau} \cap q_{3 \varepsilon}(\mathbf{j}) \neq \emptyset$; otherwise, $\mathcal{F}_{\varepsilon, \tau}^{\varphi}\left(E_{\varepsilon, \tau} \cup q_{\varepsilon}(\mathbf{j}), E^{\prime}\right)<\mathcal{F}_{\varepsilon, \tau}^{\varphi}\left(E_{\varepsilon, \tau}, E^{\prime}\right)$.

Remark 16 The regime $\tau / \varepsilon \rightarrow 0$ is completely characterized by the previous lemma. Indeed, in this case, when $\tau$ and $\varepsilon$ are small enough, $B_{4 \tau} \cap \varepsilon \mathbb{Z}^{2}=\{(0,0)\}$ and the minimizing movement is trivially $E(t) \equiv\{(0,0)\}$. This degenerate evolution is called a pinned motion. We will focus on such motions in Sect. 6 , where we will also introduce a "pinning threshold." 

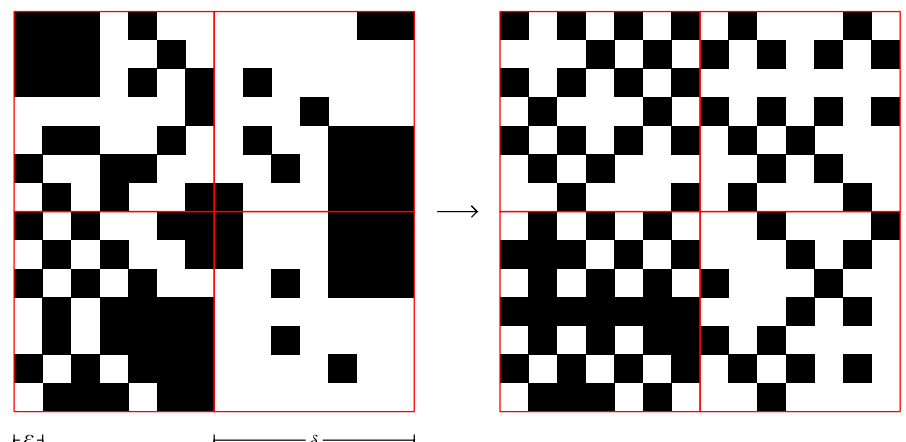

Fig. 6 On the left the set $E_{\varepsilon}$, on the right we exhibit a set $E_{\varepsilon}^{\delta}$ satisfying (i) and (ii)

\section{1 Г-Convergence of Interaction Energies}

This section is devoted to the study of the asymptotic behavior of energies $\varepsilon F_{\varepsilon}$. To this end, we associate with any admissible set $E \in \mathcal{D}_{\varepsilon}$ the corresponding characteristic function $\chi_{E} \in L^{\infty}\left(\mathbb{R}^{2}\right)$ and compute the $\Gamma$-limit with respect to the local weak ${ }^{*}$-topology. We then generalize energies in (3.1) by considering $F_{\varepsilon}: L^{\infty}\left(\mathbb{R}^{2}\right) \rightarrow$ $(-\infty,+\infty]$ as

$$
F_{\varepsilon}(u)= \begin{cases}F_{\varepsilon}(E) & u=\chi_{E}, E \in \mathcal{D}_{\varepsilon} \\ +\infty & \text { otherwise }\end{cases}
$$

with a slight abuse of notation.

Theorem 17 Let $F_{\varepsilon}$ be defined as in (4.1), and set $G_{\varepsilon}:=\varepsilon F_{\varepsilon}$. Then, $G_{\varepsilon} \Gamma$-converge as $\varepsilon \rightarrow 0$ to the energy

$$
G(u)= \begin{cases}4 \int_{\mathbb{R}^{2}}\left(\left|u(\mathbf{x})-\frac{1}{2}\right|-\frac{1}{2}\right) \mathrm{d} \mathbf{x} \quad u \in L^{\infty}\left(\mathbb{R}^{2} ;[0,1]\right) \\ +\infty & \text { otherwise, }\end{cases}
$$

with respect to the local weak ${ }^{*}$-topology.

Proof It will suffice to prove the result for $u \in L^{\infty}\left(\mathbb{R}^{2} ;[0,1]\right)$; otherwise, the assertion is trivial. We can assume, without loss of generality, that $u$ has compact support, and let $E_{\varepsilon} \in \mathcal{D}_{\varepsilon}$ be a sequence of sets such that $\chi_{E_{\varepsilon}}$ locally weakly-* converge to $u$.

We now provide a rearrangement of the centers of $E_{\varepsilon}$ which is energy decreasing. Let $\delta>0$ be fixed. We consider the lattice $\delta \mathbb{Z}^{2}$ and sets $E_{\varepsilon}^{\delta} \in \mathcal{D}_{\varepsilon}$ satisfying \# $\left(Z_{\varepsilon}\left(E_{\varepsilon}^{\delta}\right) \cap\right.$ $\left.q_{\delta}(\mathbf{i})\right)=\#\left(Z_{\varepsilon}\left(E_{\varepsilon}\right) \cap q_{\delta}(\mathbf{i})\right)$ and with the following properties:

(i) if $\varepsilon^{2} \#\left(Z_{\varepsilon}\left(E_{\varepsilon}\right) \cap q_{\delta}(\mathbf{i})\right) \leq \delta^{2} / 2$, then $Z_{\varepsilon}\left(E_{\varepsilon}^{\delta} \cap q_{\delta}(\mathbf{i})\right) \subset \varepsilon \mathbb{Z}_{e}^{2}$,

(ii) if $\varepsilon^{2} \#\left(Z_{\varepsilon}\left(E_{\varepsilon}\right) \cap q_{\delta}(\mathbf{i})\right)>\delta^{2} / 2$, then $Z_{\varepsilon}\left(E_{\varepsilon}^{\delta} \cap q_{\delta}(\mathbf{i})\right) \supset \varepsilon \mathbb{Z}_{e}^{2} \cap q_{\delta}(\mathbf{i})$,

for every $\mathbf{i} \in \delta \mathbb{Z}^{2}$ (see Fig. 6). 
Now, for every $E \in \mathcal{D}_{\varepsilon}$ and $F \in X$ we define

$$
F_{\varepsilon}(E ; F)=F_{\varepsilon}\left(E \cap E\left(\varepsilon \mathbb{Z}^{2} \cap F\right)\right),
$$

and analogously $G_{\varepsilon}(E ; F)$. In both cases (i) and (ii), we have $F_{\varepsilon}\left(E_{\varepsilon} ; q_{\delta}(\mathbf{i})\right) \geq$ $F_{\varepsilon}\left(E_{\varepsilon}^{\delta} ; q_{\delta}(\mathbf{i})\right)$. Since the contribution of the interaction between two adjacent $\delta$-squares $q_{\delta}(\mathbf{i})$ and $q_{\delta}(\mathbf{j})$ is less than $2 \delta \varepsilon$ and the number of $\delta$-squares whose intersection with $\operatorname{supp}(u) \neq \varnothing$ is proportional to $1 / \delta^{2}$, we get

$$
G_{\varepsilon}\left(E_{\varepsilon}\right) \geq G_{\varepsilon}\left(E_{\varepsilon}^{\delta}\right)-C \frac{\varepsilon}{\delta}
$$

for some positive constant $C$. Now, from the convergence of $\chi_{E_{\varepsilon}}$ to $u$, for every $\mathbf{i} \in \delta \mathbb{Z}^{2}$ we get

$$
\begin{aligned}
& G_{\varepsilon}\left(E_{\varepsilon}^{\delta} ; q_{\delta}(\mathbf{i})\right)+O(\varepsilon)=-4\left|E_{\varepsilon} \cap q_{\delta}(\mathbf{i})\right|+O(\varepsilon)=-4 \int_{q_{\delta}(\mathbf{i})} u(\mathbf{x}) \mathrm{d} \mathbf{x}:=u_{\delta}(\mathbf{i}), \\
& G_{\varepsilon}\left(E_{\varepsilon}^{\delta} ; q_{\delta}(\mathbf{i})\right)+O(\varepsilon)=-4\left|q_{\delta}(\mathbf{i}) \backslash E_{\varepsilon}\right|+O(\varepsilon)=-4 \int_{q_{\delta}(\mathbf{i})}(1-u(\mathbf{x})) \mathrm{d} \mathbf{x}:=u_{\delta}(\mathbf{i})
\end{aligned}
$$

in cases (i) and (ii), respectively. After identifying $u_{\delta}$ with its piecewise-constant interpolation, taking the limit as $\varepsilon \rightarrow 0$ first, we get

$$
\liminf _{\varepsilon \rightarrow 0} G_{\varepsilon}\left(E_{\varepsilon}\right) \geq \int_{\mathbb{R}^{2}} u_{\delta}(\mathbf{x}) \mathrm{d} \mathbf{x},
$$

and then taking the limit as $\delta \rightarrow 0$ we obtain the liminf inequality.

The construction of a recovery sequence follows an analogous argument. Let $u \in$ $L^{\infty}\left(\mathbb{R}^{2} ;[0,1]\right)$ have a compact support. Consider the lattice $\sqrt{\varepsilon} \mathbb{Z}^{2}$, and define

$$
u_{\varepsilon}(\mathbf{i})=\frac{1}{\varepsilon} \int_{q_{\sqrt{\varepsilon}}(\mathbf{i})} u(\mathbf{x}) \mathrm{d} \mathbf{x}, \quad \text { for every } \mathbf{i} \in \sqrt{\varepsilon} \mathbb{Z}^{2} .
$$

As a recovery sequence, we will choose $E_{\varepsilon}$ having the same mean (unless a small error) of $u$ in every $\sqrt{\varepsilon}$-square with maximal perimeter term. Indeed, we can take a set $E_{\varepsilon} \in \mathcal{D}_{\varepsilon}$ satisfying $\#\left(Z_{\varepsilon}\left(E_{\varepsilon}\right) \cap q_{\sqrt{\varepsilon}}(\mathbf{i})\right)=\left\lceil u_{\varepsilon}(\mathbf{i}) / \varepsilon\right\rceil$ and such that:

(i) if $u_{\varepsilon}$ (i) $\leq 1 / 2$, then $Z_{\varepsilon}\left(E_{\varepsilon}\right) \cap q_{\sqrt{\varepsilon}}$ (i) $\subset \varepsilon \mathbb{Z}_{e}^{2}$;

(ii) if $u_{\varepsilon}$ (i) $>1 / 2$, then $Z_{\varepsilon}\left(E_{\varepsilon}\right) \cap q_{\sqrt{\varepsilon}}(\mathbf{i}) \supset \varepsilon \mathbb{Z}_{e}^{2} \cap q_{\sqrt{\varepsilon}}(\mathbf{i})$.

Then, $\chi_{E_{\varepsilon}}$ weakly-* converge to $u$ and

$$
G_{\varepsilon}\left(E_{\varepsilon} ; q_{\sqrt{\varepsilon}}(\mathbf{i})\right)+O(\varepsilon)= \begin{cases}-4 \int_{q_{\sqrt{\varepsilon}}(\mathbf{i})} u(\mathbf{x}) \mathrm{d} \mathbf{x} & \text { if } u_{\varepsilon}(\mathbf{i}) \leq \frac{1}{2} \\ -4 \int_{q_{\sqrt{\varepsilon}}(\mathbf{i})}(1-u(\mathbf{x})) \mathrm{d} \mathbf{x} & \text { if } u_{\varepsilon}(\mathbf{i})>\frac{1}{2}\end{cases}
$$


for every $\mathbf{i} \in \sqrt{\varepsilon} \mathbb{Z}^{2}$, which proves that $\chi_{E_{\varepsilon}}$ is a recovery sequence and concludes the proof.

Remark 18 Note that in the proof of Theorem 17, we have exhibited a recovery sequence whose supports $E_{\varepsilon}$ also converges to $E=\operatorname{supp}(u)$ in the Hausdorff sense. This remark allows us to reduce the computation of the $\Gamma$-limit of $G_{\varepsilon}$ to functions weakly-* converging to $u$ having supports in $\mathcal{D}_{\varepsilon}$ converging to $E$ with respect to the Hausdorff distance.

Remark 19 ( $\Gamma$-limit on characteristic functions) An immediate consequence of Theorem 17 is that, among all the functions having the same support $E$, the ground state of the energy $G$ is achieved by the simple function $\frac{1}{2} \chi_{E}$. In particular, since any family of sets $\left\{E_{\varepsilon}\right\}$ converging in the Hausdorff sense to $E$ are such that $\chi_{E_{\varepsilon}}$ is weakly-* compact, from Theorem 17 we infer that

$$
\Gamma\left(d_{\mathcal{H}}\right)-\lim _{\varepsilon \rightarrow 0} G_{\varepsilon}(E)=-2|E|,
$$

once noted that the recovery sequences are $\varepsilon$-checkerboard sets.

\subsection{Convergence of the Minimizing-Movement Scheme}

We prove that when $\varepsilon / \tau \rightarrow 0$, every minimizing movement of scheme (3.5) may be seen as the solution of a continuum problem having a gradient-flow structure with respect to the limit energy. In this regime, the monotonicity constraint is not needed to obtain a completely characterized limit motion. A straightforward consequence is that the solution of the unconstrained problem corresponds to that of the monotone scheme (3.4).

Theorem 20 Let $F_{\varepsilon}, D_{\varepsilon}^{\varphi}$ and $\mathcal{F}_{\varepsilon, \tau}^{\varphi}$ be as in (3.1), (3.2) and (3.3), respectively. Then, there exists a unique minimizing movement of the unconstrained scheme (3.5) at regime $\varepsilon / \tau \rightarrow 0$ and it satisfies

$$
E(t)=B_{4 t}^{\varphi}, \quad t \geq 0
$$

Moreover, for every discrete solution $E_{\varepsilon, \tau}$ of (3.5) we have $\chi_{E_{\varepsilon, \tau}(t)} \stackrel{*}{\rightarrow} \frac{1}{2} \chi_{B_{4 t}^{\varphi}}$ for all $t \geq 0$ as $\varepsilon \rightarrow 0$.

Proof The first claim is a direct consequence of Lemma 15. Indeed, $d_{\mathcal{H}}\left(E_{\varepsilon, \tau}(t), B_{4\lfloor t / \tau\rfloor}\right)$ $<C\lfloor t / \tau\rfloor \varepsilon$, which goes to zero locally uniformly at regimes $\varepsilon / \tau \rightarrow 0$. In an analogous way as for (4.1), we further generalize the dissipations in Remark 13 as functionals $D_{\varepsilon}^{\varphi}: L^{\infty}\left(\mathbb{R}^{2}\right) \times X \rightarrow[0,+\infty]$ defined by

$$
D_{\varepsilon}^{\varphi}\left(u, E^{\prime}\right)= \begin{cases}D_{\varepsilon}^{\varphi}\left(E, E^{\prime}\right) & u=\chi_{E}, E \in \mathcal{D}_{\varepsilon} \\ +\infty & \text { otherwise. }\end{cases}
$$


Accordingly, we write $\mathcal{F}_{\varepsilon, \tau}^{\varphi}\left(u, E^{\prime}\right)=\varepsilon F_{\varepsilon}(u)+\frac{1}{\tau} D_{\varepsilon}^{\varphi}\left(u, E^{\prime}\right)$ for every $u \in L^{\infty}\left(\mathbb{R}^{2}\right)$ with $F_{\varepsilon}$ as in (4.1). Since for every sequence $\left\{E_{\varepsilon}\right\} \subset \mathcal{D}_{\varepsilon}$ such that $\chi_{E_{\varepsilon}}$ weakly-* converge to $u$ we have

$$
D_{\varepsilon}^{\varphi}\left(E_{\varepsilon}, q_{\varepsilon}\right) \rightarrow \int_{\mathbb{R}^{2}} u(\mathbf{x}) \varphi(\mathbf{x}) \mathrm{d} \mathbf{x}
$$

then Theorem 17 yields that $\mathcal{F}_{\varepsilon, \tau}^{\varphi} \Gamma$-converge, as $\varepsilon \rightarrow 0$, to the functional $\mathcal{F}_{\tau}^{\varphi}$ given by

$$
\mathcal{F}_{\tau}^{\varphi}(u):=\int_{\mathbb{R}^{2}}\left(|4 u(\mathbf{x})-2|-2+\frac{1}{\tau} u(\mathbf{x}) \varphi(\mathbf{x})\right) \mathrm{d} \mathbf{x},
$$

with respect to the weak-* topology. Energy $\mathcal{F}^{\varphi}$ has a unique minimizer in $L^{\infty}\left(\mathbb{R}^{2} ;[0,1]\right)$, given by $u=\frac{1}{2} \chi_{B_{4 \tau}^{\varphi}}$. Indeed,

$$
\begin{aligned}
\int_{\mathbb{R}^{2}}\left(|4 u(\mathbf{x})-2|-2+\frac{1}{\tau} u(\mathbf{x}) \varphi(\mathbf{x})\right) \mathrm{d} \mathbf{x}= & \int_{\{u \leq 1 / 2\}}\left(\frac{\varphi(\mathbf{x})}{\tau}-4\right) u(\mathbf{x}) \mathrm{d} \mathbf{x} \\
& +\int_{\{u>1 / 2\}}\left(4 u(\mathbf{x})-4+\frac{\varphi(\mathbf{x})}{\tau} u(\mathbf{x})\right) \mathrm{d} \mathbf{x}
\end{aligned}
$$

Both integrands are positive for almost every $\mathbf{x}$ such that $\varphi(\mathbf{x})>4 \tau$, and are minimized when $u \equiv 1 / 2$. Then, since $\Gamma$-convergence implies the convergence of minimum problems (see, for instance, Braides 2002, Theorem 1.21) and the minimum is unique, we get that $\chi_{E_{\varepsilon, \tau}^{1}}$ weakly-* converges to $u_{\tau}^{1}=\frac{1}{2} \chi_{B_{4 \tau}^{\varphi}}$ as $\varepsilon \rightarrow 0$. Note also that, by virtue of Lemma $15, E_{\varepsilon, \tau}^{1} \rightarrow B_{4 \tau}^{\varphi}$ in the Hausdorff sense and moreover by the minimality of $E_{\varepsilon, \tau}^{1}$ and Remark 13 follows that

$$
\begin{aligned}
\varepsilon F_{\varepsilon}\left(E_{\varepsilon, \tau}^{1}\right) & \leq \varepsilon F_{\varepsilon}\left(E_{\varepsilon}\right)+\frac{1}{\tau}\left(D_{\varepsilon}^{\varphi}\left(E_{\varepsilon}, q_{\varepsilon}\right)-D_{\varepsilon}^{\varphi}\left(E_{\varepsilon, \tau}^{1}, q_{\varepsilon}\right)\right) \\
& \leq \varepsilon F_{\varepsilon}\left(E_{\varepsilon}\right)+\frac{1}{\tau} \int_{\mathbb{R}^{2}}\left(\chi_{E_{\varepsilon}}(x)-\chi_{E_{\varepsilon, \tau}^{1}}(\mathbf{x})\right)(\varphi(\mathbf{x})+\varepsilon) \mathrm{d} \mathbf{x} \\
& \leq \varepsilon F_{\varepsilon}\left(E_{\varepsilon}\right)+o(1),
\end{aligned}
$$

for every $\chi_{E_{\varepsilon}}$ weakly* converging to $u_{\tau}^{1}$.

Now, we show the $\Gamma$-convergence of $\mathcal{F}_{\varepsilon, \tau}\left(\cdot, E_{\varepsilon, \tau}^{1}\right)$, which will allow us to deduce the convergence of the whole scheme by an inductive procedure. Consider $E_{\varepsilon} \in \mathcal{D}_{\varepsilon}$ such that $\chi_{E_{\varepsilon}}$ are converging weakly-* to some $u \in L^{\infty}\left(\mathbb{R}^{2}\right)$. Mimicking the arguments of the proof of Theorem 17, we consider $E_{\varepsilon}^{\prime} \in \mathcal{D}_{\varepsilon}$ satisfying $\#\left(Z_{\varepsilon}\left(E_{\varepsilon}^{\prime}\right) \cap q_{\sqrt{\varepsilon}}(\mathbf{i})\right)=$ $\#\left(Z_{\varepsilon}\left(E_{\varepsilon}\right) \cap q_{\sqrt{\varepsilon}}(\mathbf{i})\right)$ and such that:

(i) if \# $\left(Z_{\varepsilon}\left(E_{\varepsilon}\right) \cap q_{\sqrt{\varepsilon}}(\mathbf{i})\right) \leq \#\left(Z_{\varepsilon}\left(E_{\varepsilon, \tau}^{1}\right) \cap q_{\sqrt{\varepsilon}}(\mathbf{i})\right)$, then $Z_{\varepsilon}\left(E_{\varepsilon}^{\prime} \cap q_{\sqrt{\varepsilon}}(\mathbf{i})\right) \subset Z_{\varepsilon}\left(E_{\varepsilon, \tau}^{1}\right)$,

(ii) if \# $\left(Z_{\varepsilon}\left(E_{\varepsilon}\right) \cap q_{\sqrt{\varepsilon}}(\mathbf{i})\right)>\#\left(Z_{\varepsilon}\left(E_{\varepsilon, \tau}^{1}\right) \cap q_{\sqrt{\varepsilon}}(\mathbf{i})\right)$, then $Z_{\varepsilon}\left(E_{\varepsilon}^{\prime} \cap q_{\sqrt{\varepsilon}}(\mathbf{i})\right) \supset Z_{\varepsilon}\left(E_{\varepsilon, \tau}^{1}\right) \cap$ $q \sqrt{\varepsilon}(\mathbf{i})$ 
for every $\mathbf{i} \in \sqrt{\varepsilon} \mathbb{Z}^{2} \cap B_{4 \tau}^{\varphi}$, and $Z_{\varepsilon}\left(E_{\varepsilon}^{\prime}\right) \backslash B_{4 \tau}^{\varphi}=Z_{\varepsilon}\left(E_{\varepsilon}\right) \backslash B_{4 \tau}^{\varphi}$. Reasoning as in the proof of Theorem 17 and from (4.5), $\chi_{E_{\varepsilon}^{\prime}}$ still weakly-* converges to $u$ and $\varepsilon F_{\varepsilon}\left(E_{\varepsilon}\right)+o(1) \geq$ $\varepsilon F_{\varepsilon}\left(E_{\varepsilon}^{\prime}\right)$. Then, we get

$$
\begin{aligned}
\mathcal{F}_{\varepsilon, \tau}^{\varphi}\left(E_{\varepsilon}, E_{\varepsilon, \tau}^{1}\right)+o(1) \geq & \mathcal{F}_{\varepsilon, \tau}^{\varphi}\left(E_{\varepsilon}^{\prime}, E_{\varepsilon, \tau}^{1}\right) \\
= & \varepsilon F_{\varepsilon}\left(E_{\varepsilon}^{\prime}\right)+\frac{1}{\tau} D_{\varepsilon}^{\varphi}\left(E_{\varepsilon} \backslash B_{4 \tau}^{\varphi}, E_{\varepsilon, \tau}^{1}\right) \\
& +\frac{1}{\tau} \sum_{i \in \sqrt{\varepsilon} \mathbb{Z}^{2} \cap B_{4 \tau}^{\varphi}} D_{\varepsilon}^{\varphi}\left(E_{\varepsilon}^{\prime} \cap q_{\sqrt{\varepsilon}}(\mathbf{i}), E_{\varepsilon, \tau}^{1}\right) .
\end{aligned}
$$

Since $D_{\varepsilon}^{\varphi}\left(E_{\varepsilon}^{\prime} \cap q_{\sqrt{\varepsilon}}(\mathbf{i}), E_{\varepsilon, \tau}^{1}\right)=C \varepsilon^{3}\left|\# Z_{\varepsilon}\left(E_{\varepsilon}\right)-\# Z_{\varepsilon}\left(E_{\varepsilon, \tau}^{1}\right)\right|+O\left(\varepsilon^{2}\right)$ and $d_{\varepsilon}^{\varphi}\left(\mathbf{x}, \partial E_{\varepsilon, \tau}^{1}\right)$ converge uniformly to $d^{\varphi}\left(\mathbf{x}, B_{4 \tau}^{\varphi}\right)$ for every $\mathbf{x} \notin E_{\tau}^{1}$, we get that

$$
\Gamma-\lim _{\varepsilon \rightarrow 0} \mathcal{F}_{\varepsilon, \tau}^{\varphi}\left(u, E_{\varepsilon, \tau}^{1}\right)=\int_{\mathbb{R}^{2}}\left(|4 u(\mathbf{x})-2|-2+\frac{1}{\tau} u(\mathbf{x}) d^{\varphi}\left(\mathbf{x}, B_{4 \tau}^{\varphi}\right)\right) \mathrm{d} \mathbf{x},
$$

since the same argument applies to every recovery sequence $E_{\varepsilon}$. By arguing as above, we get $\chi_{E_{\varepsilon, \tau}^{2}}$ converge to $1 / 2 \chi_{B_{8 \tau}^{\varphi}}$ and by induction the result follows.

Arguing as in the proof of Theorem 20, we obtain the following result.

Corollary 21 Let $F_{\varepsilon}, D_{\varepsilon}^{\varphi}$ and $\mathcal{F}_{\varepsilon, \tau}^{\varphi}$ be defined as in (3.1)-(3.3). Then, there exists a unique minimizing movement of scheme (3.4) at regime $\varepsilon / \tau \rightarrow 0$ and it satisfies $E(t)=B_{4 t}^{\varphi}$ for $t \geq 0$. Moreover, for every discrete solution $E_{\varepsilon, \tau}$ of (3.4) we have $\chi_{E_{\varepsilon, \tau}(t)} \stackrel{*}{\rightarrow} \frac{1}{2} \chi_{B_{4 t}^{\varphi}}$ for $t \geq 0$ as $\varepsilon \rightarrow 0$.

Remark 22 Arguing as in Remark 19, for any $E^{\prime} \in \mathcal{X}$ and every $E_{\varepsilon}^{\prime}$ converging to $E^{\prime}$ in $d_{\mathcal{H}}$ such that $\varepsilon F\left(E_{\varepsilon}^{\prime}\right) \rightarrow-2\left|E^{\prime}\right|$ we get, from (4.6), that

$$
\Gamma\left(d_{\mathcal{H}}\right)-\lim _{\varepsilon \rightarrow 0} \mathcal{F}_{\varepsilon, \tau}^{\varphi}\left(E, E_{\varepsilon}^{\prime}\right)=\mathcal{F}_{\tau}^{\varphi}\left(E, E^{\prime}\right):=-2|E|+\frac{1}{2 \tau} \int_{E \Delta E^{\prime}} d^{\varphi}\left(x, E^{\prime}\right) \mathrm{d} x .
$$

Note that the minima of $\mathcal{F}_{\tau}^{\varphi}\left(\cdot, E^{\prime}\right)$ are solutions of

$$
\left(-2+\frac{1}{2 \tau} d^{\varphi}\left(x, E^{\prime}\right)\right) v_{E}(x) \mathcal{H}^{1}\llcorner\partial E=0 ;
$$

that is, $E \in \mathcal{X}$ such that $d^{\varphi}\left(x, E^{\prime}\right) \equiv 4 \tau$ for $\mathcal{H}^{1}$-almost every $x \in \partial E$. This gives that the limit scheme

$$
\left\{\begin{array}{l}
E_{\tau}^{0}=\{(0,0)\}, \\
E_{\tau}^{k+1} \in \underset{E \in X}{\operatorname{argmin}} \mathcal{F}_{\tau}^{\varphi}\left(E, E_{\tau}^{k}\right)
\end{array}\right.
$$

is solved by $E_{\tau}^{k}=B_{4 k \tau}^{\varphi}$. Hence, by Theorem 20 and Corollary 21 the minimizing movements of schemes (3.4) and (3.5) at regimes $\varepsilon / \tau \rightarrow 0$ are solutions of limit scheme (4.7). 


\section{The Critical Regime: A Microscopic Checkerboard Structure}

So far, we have shown that scheme (3.4) is completely characterized in the regimes $\tau / \varepsilon \rightarrow 0$ (Remark 16) and $\varepsilon / \tau \rightarrow 0$ (Remark 22). Throughout this section, we will study the regimes where $\varepsilon / \tau$ has a nonzero finite limit, which turn out to be richer of features than the others.

Without loss of generality, we consider only the case $\varepsilon=\alpha \tau$, where $\alpha>0$ is a positive constant. The main goal is to determine any solution to the iterative variational scheme (3.4). Within this regime, instead of solving a family of schemes depending on $\varepsilon$, by a rescaling argument we can solve one minimization scheme in the unique environment $\mathbb{Z}^{2}$. Indeed, for every $E, F \in \mathcal{D}_{\varepsilon}$, the energies defined in (3.3) can be rewritten as

$$
\begin{aligned}
\mathcal{F}_{\varepsilon, \tau}^{\varphi}(E, F) & =-\varepsilon \mathcal{H}^{1}(\partial E)+\frac{1}{\tau} D_{\varepsilon}^{\varphi}(E, F)=-\varepsilon \mathcal{H}^{1}(E)+\frac{\varepsilon^{2}}{\tau} \sum_{i \in Z_{\varepsilon}(E) \triangle Z_{\varepsilon}(F)} d_{\varepsilon}^{\varphi}(\mathbf{i}, \partial F) \\
& =\varepsilon\left(-\mathcal{H}^{1}(\partial E)+\alpha \sum_{\mathbf{i} \in Z_{\varepsilon}(E) \triangle Z_{\varepsilon}(F)} d_{\varepsilon}^{\varphi}(\mathbf{i}, \partial F)\right)=\varepsilon^{2} \mathcal{F}_{\alpha}^{\varphi}\left(\frac{1}{\varepsilon} E, \frac{1}{\varepsilon} F\right),
\end{aligned}
$$

where we have defined $\mathcal{F}_{\alpha}^{\varphi}: \mathcal{D} \times \mathcal{D} \rightarrow \mathbb{R}$ as

$$
\mathcal{F}_{\alpha}^{\varphi}\left(E^{\prime}, F^{\prime}\right)=-\mathcal{H}^{1}\left(\partial E^{\prime}\right)+\alpha \sum_{\mathbf{i} \in Z\left(E^{\prime}\right) \triangle Z\left(F^{\prime}\right)} d^{\varphi}\left(\mathbf{i}, \partial F^{\prime}\right)
$$

Thus, the solutions of (3.4) are $E_{\varepsilon, \tau}^{k}=\varepsilon E_{\alpha}^{k}$ for every $\varepsilon>0, k \in \mathbb{N}$, where $\left\{E_{\alpha}^{k}\right\}$ solves the scaled scheme

$$
\left\{\begin{array}{l}
E_{\alpha}^{0}=q, \\
E_{\alpha}^{k+1} \in \underset{E \in \mathcal{D}, E \supset E_{\alpha}^{k}}{\operatorname{argmin}} \mathcal{F}_{\alpha}\left(E, E_{\alpha}^{k}\right), \quad k \geq 0 .
\end{array}\right.
$$

We will prove that scheme (5.2) has a unique solution $\left\{E_{\alpha}^{k}\right\}$ whenever $\alpha$ is outside a countable set (see Remark 24). If $\alpha$ is greater than a threshold value $\tilde{\alpha}>0$, the corresponding solution is trivially $E_{\alpha}^{k} \equiv q$, and we will say that the motion is pinned. If instead $\alpha$ is below the pinning threshold (see Definition 40 ), the solutions $\left\{E_{\alpha}^{k}\right\}$ have a checkerboard structure; that is, $E_{\alpha}^{k} \in \mathcal{A}^{e}$ for every $k \in \mathbb{N}$, and they are obtained by the iterative formula

$$
Z\left(E_{\alpha}^{k+1}\right)=Z\left(E_{\alpha}^{k}\right)+Z\left(E_{\alpha}^{1}\right), \quad \text { for every } k \in \mathbb{N}, k \geq 1
$$

We call this process nucleation from the origin, and the lattice set $Z\left(E_{\alpha}^{1}\right)$, which we call the nucleus of the process, completely characterizes the motion. The limit evolution will be a motion of expanding polygons with constant velocity; both the velocity and the shape of the limit sets will be a discretization (depending on $\alpha$ ) of those of the minimizing movement of (3.4) at regime $\varepsilon / \tau \rightarrow 0$ studied in Sect. 4 . This result will 
be proven under a technical assumption on the "convexity" of the nucleus $Z\left(E_{\alpha}^{1}\right)$ (cf. (5.13)) which will allow us to use a localization method to solve any minimization problem of the scheme (5.2).

The following result is a rereading of Lemma 15 in the scaled setting. We note that, as for Lemma 15, the following result holds for every norm.

Lemma 23 Let $\mathcal{F}_{\alpha}^{\varphi}: \mathcal{D} \times \mathcal{D} \rightarrow \mathbb{R}$ be as in (5.1), where $\mathcal{D}$ is defined as in (2.1) with $\varepsilon=1$. Then, for any given $E^{\prime} \in \mathcal{D}$ it holds that

$$
\mathcal{F}_{\alpha}^{\varphi}\left(E(\mathcal{J}), E^{\prime}\right) \leq \mathcal{F}_{\alpha}^{\varphi}\left(E, E^{\prime}\right), \quad \text { where } \mathcal{J}=\left\{\mathbf{i} \in Z(E): d^{\varphi}\left(\mathbf{i}, \partial E^{\prime}\right) \leq \frac{4}{\alpha}\right\}
$$

for every $E \in \mathcal{D}$. In particular, for every $\left\{E_{\alpha}^{k}\right\}$ discrete solution of the scheme (5.2), there holds

$$
Z\left(E_{\alpha}^{k+1}\right) \subset\left\{\mathbf{i} \in \mathbb{Z}^{2}: d^{\varphi}\left(\mathbf{i}, \partial E_{\alpha}^{k}\right) \leq \frac{4}{\alpha}\right\}, \quad \text { for every } k \in \mathbb{N} .
$$

Proof The result immediately follows from the fact that for every $E^{\prime} \in \mathcal{D}$, the variation of adding an isolated square to any $E \in \mathcal{D}$ is $\mathcal{F}_{\alpha}^{\varphi}\left(E \cup q(\mathbf{i}), E^{\prime}\right)-\mathcal{F}_{\alpha}^{\varphi}\left(E, E^{\prime}\right)=$ $-4+\alpha d^{\varphi}\left(\mathbf{i}, \partial E^{\prime}\right)$.

Remark 24 (Non-uniqueness) Note that for every $\mathbf{i} \in \mathbb{Z}^{2}$ such that $d^{\varphi}\left(\mathbf{i}, \partial E_{\alpha}^{k}\right)=\frac{4}{\alpha}$ (if any), the energy contribution of the square $q(\mathbf{i})$ is zero; that is,

$$
\mathcal{F}_{\alpha}^{\varphi}\left(E_{\alpha}^{k+1} \cup q(\mathbf{i}), E_{\alpha}^{k}\right)=\mathcal{F}_{\alpha}^{\varphi}\left(E_{\alpha}^{k+1} \backslash q(\mathbf{i}), E_{\alpha}^{k}\right) .
$$

Therefore, in this case, there is non-uniqueness of solutions for the problem (5.2). Note that if $\varphi(\mathbf{x})=\frac{4}{\alpha}$ has no integer solutions, then, by the periodicity of $\mathbb{Z}^{2}$, the same holds true for equation $d^{\varphi}(\mathbf{x}, \partial E)=\frac{4}{\alpha}$ for every $E \in \mathcal{D}$. This in particular implies that the $k$ th minimization problem of the scheme (5.2) has non-unique solution if and only if the first minimization problem has non-unique solution.

With the previous remark in mind, we define the singular set $\Lambda^{\varphi}$ as

$$
\Lambda^{\varphi}:=\left\{\frac{4}{\varphi(\mathbf{i})}: \mathbf{i} \in \mathbb{Z}_{e}^{2} \backslash\{(0,0)\}\right\}
$$

Note that the set $\Lambda^{\varphi}$ is countable and has a unique accumulation point in 0 .

Example 25 We take $\varphi$ as the $\ell^{\infty}$-norm and choose $\alpha=4$, so that $\alpha \in \Lambda^{\varphi}$ as defined in (5.4). In this case, the set of lattice points having zero energy is $\left\{\mathbf{i} \in \mathbb{Z}^{2}:\|i\|_{\infty}=1\right\}$. This yields that $\mathcal{F}_{\alpha}^{\varphi}(q, q)=\mathcal{F}_{\alpha}^{\varphi}(E, q)=-4$ for every admissible set $E \subset q \cup\{q(\mathbf{i})$ : $\left.\left|i_{1}\right|=\left|i_{2}\right|=1\right\}$ which implies that the minimum of the first step of (5.2) is not unique. As already noted in Remark 24, the same situation arises at each minimization step of the scheme (5.2). 

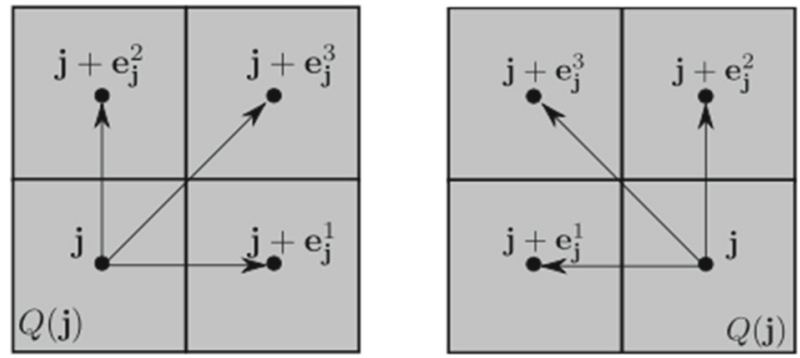

Fig. 7 Examples of $2 \times 2$ squares of the covering. On the left the case $j_{1}, j_{2}>0$, on the right $j_{1}>0$, $j_{2}<0$

Without entering into the details, we may check that every parametrized family $E:[0,+\infty) \rightarrow X$ of connected sets satisfying

$$
E(0)=\{(0,0)\}, \quad E(t) \subset E(s) \text { for every } t<s, \quad\left\|v_{\perp}(t)\right\|_{\infty} \leq 4 \text { for every } t \geq 0,
$$

is a minimizing movement, where $v_{\perp}$ denotes the normal velocity of $\partial E(t)$. Indeed, for every fixed $t>0$, from $(5.5)$ we have $E(t) \subseteq[-4 t, 4 t]^{2}$, since $E(t)$ is connected. Then, for any $\tau>0$ define

$$
E_{\varepsilon, \tau}^{k}:=E\left(E(k \tau) \cap \varepsilon \mathbb{Z}_{e}^{2}\right)
$$

Since $E(k \tau) \subseteq[-4 k \tau, 4 k \tau]^{2}=[-k \varepsilon, k \varepsilon]^{2}, E_{\tau, \varepsilon}^{k}$ can be obtained by solving the first $k$ steps of (3.4). The corresponding discrete solutions $E_{\varepsilon, \tau}(t)$ converge to $E(t)$ as $\varepsilon, \tau \rightarrow 0$ in the Hausdorff sense for every $t>0$, whence $E(t)$ is a minimizing movement.

\subsection{A Localization Argument: The 2 × 2-Square Tiling}

In order to determine the optimal structure of a minimizer, we will argue locally by defining the following covering of admissible sets.

Definition $26\left(2 \times 2\right.$-square coverings) For every $\mathbf{j}=\left(j_{1}, j_{2}\right) \in \mathbb{Z}^{2}$, we define the vectors $\mathbf{e}_{\mathbf{j}}^{1}=\left(\operatorname{sgn}\left(j_{1}\right), 0\right), \mathbf{e}_{\mathbf{j}}^{2}=\left(0, \operatorname{sgn}\left(j_{2}\right)\right), \mathbf{e}_{\mathbf{j}}^{3}=\mathbf{e}_{\mathbf{j}}^{1}+\mathbf{e}_{\mathbf{j}}^{2}$ and, correspondingly, the $2 \times 2$ square (see Fig. 7)

$$
Q(\mathbf{j}):=q(\mathbf{j}) \cup \bigcup_{k=1}^{3} q\left(\mathbf{j}+\mathbf{e}_{\mathbf{j}}^{k}\right)
$$

Let $E \in \mathcal{D}$ be an admissible set. Then, we define the family of sets

$$
\mathcal{S}_{e}(E):=\left\{Q(\mathbf{j}): \mathbf{j} \in \mathbb{Z}_{e}^{2} \text { with } j_{1}, j_{2} \text { odd, } Q(\mathbf{j}) \cap E \neq \emptyset\right\},
$$


Fig. 8 The picture clarifies the $2 \times 2$-square covering for a set $E$, whose boundary is marked by a bold black line. The darker $2 \times 2$ squares are in $\mathcal{S}_{e}^{c}(E)$, the lighter ones in $\mathcal{S}_{e}^{b}(E)$. The areas in white are those left uncovered

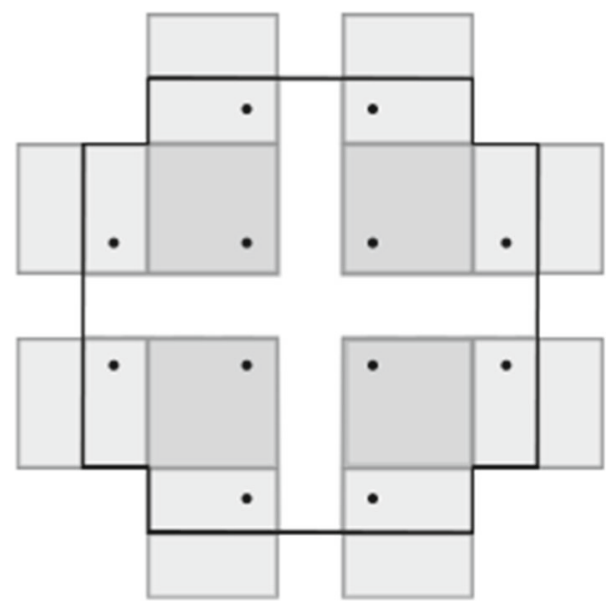

which is a covering of non-overlapping squares of $E \backslash \bigodot_{0}$, where $\bigodot_{0}:=\bigcup\left\{q(\mathbf{i}) \mid i_{1} i_{2}=\right.$ $0\}$ (see Fig. 8). We can subdivide the squares of $\mathcal{S}_{e}(E)$ in those contained in $E$ and those that are not, defining the partition $\mathcal{S}_{e}(E)=\mathcal{S}_{e}^{b}(E) \cup \mathcal{S}_{e}^{c}(E)$ where $\mathcal{S}_{e}^{c}(E)=$ $\left\{Q(\mathbf{j}) \in \mathcal{S}_{e}(E) \mid Q(\mathbf{j}) \subseteq E\right\}$ and $\mathcal{S}_{e}^{b}(E)=\left\{Q(\mathbf{j}) \in \mathcal{S}_{e}(E) \mid Q(\mathbf{j}) \cap E^{c} \neq \emptyset\right\}$.

\subsection{Choice of the Dissipation Term}

We restrict our analysis to dissipations (3.2) induced by an absolute norm $\varphi$; i.e., $\varphi(\mathbf{x})$ depends only on $\left|x_{1}\right|$ and $\left|x_{2}\right|$, with the additional assumptions

(H1) $\varphi$ is symmetric (or permutation invariant); that is, $\varphi\left(x_{1}, x_{2}\right)=\varphi\left(x_{2}, x_{1}\right)$ for every $\mathbf{x} \in \mathbb{R}^{2}$

(H2) $\varphi$ complies with the normalization condition $\varphi(1,0)=\varphi(0,1)=1$.

We refer to an absolute norm with these properties as a symmetric absolute normalized norm. The $\ell^{p}$-norms, $1 \leq p \leq \infty$, are examples of such norms. This choice is of course motivated by the symmetry properties of the corresponding unit balls, which simplify the computations and the arguments of the proofs. Moreover, as remarked in Sect. 2.4 an absolute norm is a submodular function on $\mathbb{R}^{2+}$, a property that will be crucial in the sequel as it will allow to reduce the main minimization problem to a finite number of local minimization problems, taking into account four-point interactions. Indeed, we can infer from (2.10) a submodularity-type inequality involving only the norms of the four lattice points contained in any of the $2 \times 2$ squares of the coverings defined above. Namely,

$$
\varphi(\mathbf{i})+\varphi\left(\mathbf{i}+\mathbf{e}_{\mathbf{i}}^{3}\right) \leq \varphi\left(\mathbf{i}+\mathbf{e}_{\mathbf{i}}^{1}\right)+\varphi\left(\mathbf{i}+\mathbf{e}_{\mathbf{i}}^{2}\right),
$$

for every $\mathbf{i} \in \mathbb{Z}^{2}$. 

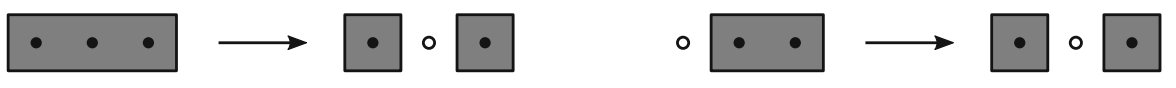

Fig. 9 Clusters of two or three lattice points are "locally" not energetically convenient

\subsection{The First Step of the Evolution: Checkerboards Nucleating from a Point}

With the covering argument of Sect. 5.1 and the key norm inequality (5.8) at hand, we are now in position to give the explicit characterization of the first step $E_{\alpha}^{1}$ of the discrete evolution, showing that it is an even checkerboard. A local analysis by means of the $2 \times 2$-square tilings will allow us to prove, with Proposition 27, that the set of centers of $E_{\alpha}^{1}$ coincides with the discretization of the ball $B_{\frac{4}{\alpha}}$ on the even lattice $\mathbb{Z}_{e}^{2}$. We stress the generality of the following result, which only requires $\varphi$ to be an absolute norm without any additional assumption; in particular, we do not assume (H1) and (H2).

Proposition 27 Let $\varphi$ be an absolute norm, let $\alpha>0$ be such that $\alpha \notin \Lambda^{\varphi}$, and let $\mathcal{F}_{\alpha}^{\varphi}$ be as in (5.1). Then, the first minimization problem of scheme (5.2) has a unique solution

$$
E_{\alpha}^{1}=\underset{E \in \mathcal{D}, E \supset q}{\operatorname{argmin}} \mathcal{F}_{\alpha}^{\varphi}(E, q)
$$

and it satisfies

$$
E_{\alpha}^{1}=E\left(\mathbb{Z}_{e}^{2} \cap B_{\frac{4}{\alpha}}^{\varphi}\right)
$$

In particular, $E_{\alpha}^{1} \in \mathcal{A}_{\mathrm{conv}}^{e}$.

Proof The argument does not require the normalization assumption (H2); we then set

$$
\varphi_{\max }:=\max \{\varphi(1,0), \varphi(0,1)\}, \quad \varphi_{\min }:=\min \{\varphi(1,0), \varphi(0,1)\}
$$

and we assume, without loss of generality, that $\varphi_{\max }=\varphi(1,0)$. Note that $\frac{4}{\varphi_{\min }}, \frac{4}{\varphi_{\max }} \in$ $\Lambda^{\varphi}$.

If $\alpha>\frac{4}{\varphi_{\min }}$, we get $E_{\alpha}^{1}=q$ since $\mathcal{F}_{\alpha}^{\varphi}(q(\mathbf{i}), q)>0$ for every $\mathbf{i} \in \mathbb{Z}^{2} \backslash\{(0,0)\}$ and (5.9) trivially holds. If $\frac{4}{\varphi_{\max }}<\alpha<\frac{4}{\varphi_{\min }}$, we get that for any $\mathbf{i}=\left(i_{1}, i_{2}\right)$ with $i_{1} \neq 0$, there holds $\mathcal{F}_{\alpha}^{\varphi}(q(\mathbf{i}), q)>0$, thus $Z\left(E_{\alpha}^{1}\right) \subset\{0\} \times \mathbb{Z}$.

Let $E \in \mathcal{D}$ be a competitor such that $Z(E) \subset\{0\} \times \mathbb{Z}$. If $\mathbf{i} \in Z(E) \backslash\{(0,0)\}$ has two nearest-neighbors, removing $q(\mathbf{i})$ leaves the total perimeter unchanged but decreases the dissipation (see Fig. 9). If instead $\mathbf{i}$ has only one nearest-neighbor $\mathbf{i}^{\prime} \neq(0,0)$ and if $\left|i_{2}\right|<\left|i_{2}^{\prime}\right|$, then shifting $q(\mathbf{i})$ toward the origin does not decrease the perimeter but reduces the dissipation; if instead $\left|i_{2}\right|>\left|i_{2}^{\prime}\right|$, the same holds shifting $q\left(\mathbf{i}^{\prime}\right)$ (see Fig. 9). Hence, we may restrict our analysis to the two configurations $E\left(\mathbb{Z}_{e}^{2} \cap B_{\frac{4}{\alpha}}^{\varphi}\right)$ and $E\left(\mathbb{Z}_{o}^{2} \cap B_{\frac{4}{\alpha}}^{\varphi}\right) \cup q$. A comparison between the two energy contributions yields 
Fig. 10 It is convenient to remove $q(\mathbf{i})$ if $\mathbf{i}$ has two nearest-neighbors
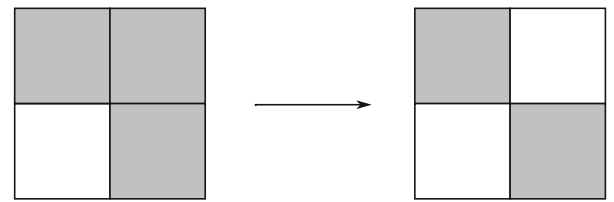

that the variation from the odd checkerboard to the even one is less than 0 ; thus, $E_{\alpha}^{1}=E\left(\mathbb{Z}_{e}^{2} \cap B_{\frac{4}{\alpha}}^{\varphi}\right)$.

Now, let $\alpha<\frac{4}{\varphi_{\max }}$. We consider the covering described in Definition 26. First, we note that the energy of every admissible set $E$ complies with the estimate

$$
\mathcal{F}_{\alpha}^{\varphi}(E, q) \geq \sum_{Q(\mathbf{j}) \in \mathcal{S}_{e}\left(\mathbb{R}^{2}\right)} \mathcal{F}_{\alpha}^{\varphi}(E \cap Q(\mathbf{j}), q)+\mathcal{F}_{\alpha}^{\varphi}\left(E \cap \mathcal{C}_{0}, q\right)
$$

the equality holding if and only if $\{E \cap Q(\mathbf{j})\}$ and $E \cap \mathcal{C}_{0}$ are non-overlapping; this is the case of sets $E$ having a checkerboard structure. Inequality (5.10) corresponds to localizing the energy, neglecting interactions between neighboring squares.

From Lemma 23, we can reduce our analysis to admissible sets contained in $E_{\alpha, \varphi}:=$ $\mathbb{Z}^{2} \cap B_{\frac{4}{\alpha}}^{\varphi}$ and inequality (5.10) holds restricting the sum to every $Q(\mathbf{j}) \in \mathcal{S}_{e}\left(E_{\alpha, \varphi}\right)$ since $\mathcal{F}_{\alpha}^{\stackrel{\alpha}{\varphi}}(q(\mathbf{j}), q)>0$ for every $\varphi(\mathbf{j})>\frac{4}{\alpha}$. We will prove that

$$
\begin{gathered}
\left.\min _{E \in \mathcal{D}, E \supset q} \mathcal{F}_{\alpha}^{\varphi}(E \cap Q(\mathbf{j}), q)=\mathcal{F}_{\alpha}^{\varphi}\left(E\left(\mathbb{Z}_{e}^{2} \cap B_{\frac{4}{\alpha}}^{\varphi}\right)\right) \cap Q(\mathbf{j}), q\right) \\
\min _{E \in \mathcal{D}, E \supset q} \mathcal{F}_{\alpha}^{\varphi}\left(E \cap \mathcal{C}_{0}, q\right)=\mathcal{F}_{\alpha}^{\varphi}\left(E\left(\mathbb{Z}_{e}^{2} \cap B_{\frac{4}{\alpha}}^{\varphi}\right) \cap \mathcal{C}_{0}, q\right)
\end{gathered}
$$

for every $Q(\mathbf{j}) \in \mathcal{S}_{e}\left(E_{\alpha, \varphi}\right)$; that is, the optimal structure is an even checkerboard set in each of the following cases: (a) inside $Q(\mathbf{j}) \in \mathcal{S}_{e}^{c}\left(E_{\alpha, \varphi}\right)$; (b) inside $Q(\mathbf{j}) \in \mathcal{S}_{e}^{b}\left(E_{\alpha, \varphi}\right)$; (c) on $E_{\alpha, \varphi} \cap \mathcal{C}_{0}$. In the sequel, $E$ will denote a general competitor $E \in \mathcal{D}, E \subset E_{\alpha, \varphi}$.

(a) Consider $Q(\mathbf{j}) \in \mathcal{S}_{e}^{c}\left(E_{\alpha, \varphi}\right)$ and let $q(\mathbf{i}) \subset Q(\mathbf{j}) \cap E$. Note that the class $\mathcal{S}_{e}^{c}(E)$ is not empty if and only if $\alpha<\frac{\varphi_{\max }}{2}$. Moreover, since adding an isolated square in $Q(\mathbf{j})$ is always energetically convenient, we can restrict to configurations of $Q(\mathbf{j}) \cap$ $E$ consisting of exactly two squares $q\left(\mathbf{i}^{\prime}\right)$ and $q\left(\mathbf{i}^{\prime \prime}\right)$ (see Fig. 10). Now, if $q\left(\mathbf{i}^{\prime}\right) \cup$ $q\left(\mathbf{i}^{\prime \prime}\right)$ has no checkerboard structure; that is, $\mathbf{i}^{\prime}$ and $\mathbf{i}^{\prime \prime}$ are nearest-neighbors, both the checkerboard configurations $E^{\prime}$ and $E^{\prime \prime}$, containing $q\left(\mathbf{i}^{\prime}\right)$ and $q\left(\mathbf{i}^{\prime \prime}\right)$, respectively, decrease the energy. Indeed, the corresponding variation of the energy is given by

$$
\begin{aligned}
& \mathcal{F}_{\alpha}^{\varphi}\left(E^{\prime}, q\right)-\mathcal{F}_{\alpha}^{\varphi}\left(q\left(\mathbf{i}^{\prime}\right) \cup q\left(\mathbf{i}^{\prime \prime}\right), q\right) \leq-2+\alpha \varphi_{\max }, \\
& \mathcal{F}_{\alpha}^{\varphi}\left(E^{\prime \prime}, q\right)-\mathcal{F}_{\alpha}^{\varphi}\left(q\left(\mathbf{i}^{\prime}\right) \cup q\left(\mathbf{i}^{\prime \prime}\right), q\right) \leq-2+\alpha \varphi_{\max } .
\end{aligned}
$$

This variation is never positive, since when $\alpha>\frac{2}{\varphi_{\max }}$ the class $\mathcal{S}_{e}\left(E_{\alpha, \varphi}\right)$ is empty. Thus, any checkerboard configuration inside $Q(\mathbf{j})$ is a competitor with less energy than $E$ (see Fig. 11). Now, we should compare the energies of the two possible checkerboard configurations inside $Q(\mathbf{j})$. For this, we note that the variation of the energy in order 


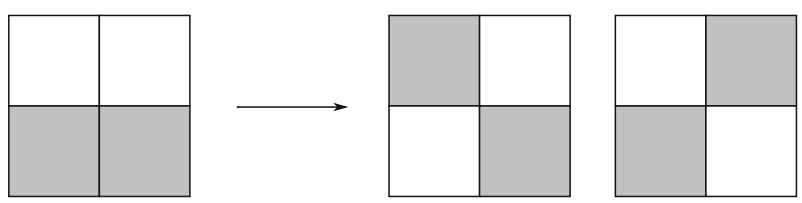

Fig. 11 Any checkerboard configuration inside $Q(\mathbf{j})$ is a competitor with less energy
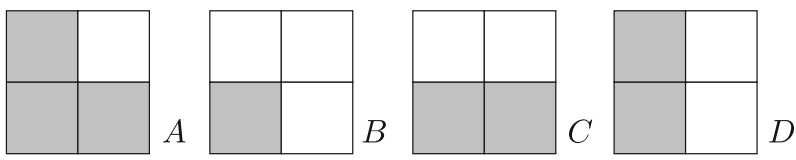

Fig. 12 The possible cases of $Q(\mathbf{j}) \cap E_{\alpha, \varphi}$

to pass from the odd checkerboard configuration $q\left(\mathbf{j}+\mathbf{e}_{\mathbf{j}}^{1}\right) \cup q\left(\mathbf{j}+\mathbf{e}_{\mathbf{j}}^{2}\right)$ to the even one $q(\mathbf{j}) \cup q\left(\mathbf{j}+\mathbf{e}_{\mathbf{j}}^{3}\right)$ is

$$
\begin{aligned}
& \mathcal{F}_{\alpha}^{\varphi}\left(q(\mathbf{j}) \cup q\left(\mathbf{j}+\mathbf{e}_{\mathbf{j}}^{3}\right), q\right)-\mathcal{F}_{\alpha}^{\varphi}\left(q\left(\mathbf{j}+\mathbf{e}_{\mathbf{j}}^{1}\right) \cup q\left(\mathbf{j}+\mathbf{e}_{\mathbf{j}}^{2}\right), q\right) \\
& =\alpha\left(\varphi(\mathbf{j})+\varphi\left(\mathbf{j}+\mathbf{e}_{\mathbf{j}}^{3}\right)-\varphi\left(\mathbf{j}+\mathbf{e}_{\mathbf{j}}^{1}\right)-\varphi\left(\mathbf{j}+\mathbf{e}_{\mathbf{j}}^{2}\right)\right),
\end{aligned}
$$

which is non-positive by (5.8).

(b) Now, let $Q(\mathbf{j}) \in \mathcal{S}_{e}^{b}\left(E_{\alpha, \varphi}\right)$. Without loss of generality, we may assume that $j_{1}, j_{2}>0$, the situation being completely symmetric in the other cases. Inside such a $2 \times 2$ square, we have four possible cases for $Q(\mathbf{j}) \cap E_{\alpha, \varphi}$, as pictured in Fig. 12. We claim that the configuration with minimal energy inside $Q(\mathbf{j})$ is a checkerboard set. Consider first $\alpha>\frac{2}{\varphi_{\max }}$, then $\mathbf{i} \in B_{\frac{4}{\alpha}}^{\varphi}$ if and only if $\left|i_{1}\right| \leq 1$; thus, the only possible cases for $Q(\mathbf{j}) \cap E_{\alpha, \varphi}$ are those labeled by $B$ and $D$ in Fig. 12. Since

$\mathcal{F}_{\alpha}^{\varphi}(q(\mathbf{j}), q)<\mathcal{F}_{\alpha}^{\varphi}\left(\mathbf{e}_{\mathbf{j}}^{2}, q\right), \quad \mathcal{F}_{\alpha}^{\varphi}(q(\mathbf{j}), q)-\mathcal{F}_{\alpha}^{\varphi}\left(q(\mathbf{j}) \cup q\left(\mathbf{j}+\mathbf{e}_{\mathbf{j}}^{2}\right), q\right)=2-\alpha \varphi_{\max }<0$

in both cases the optimal configuration is $q(\mathbf{j})$. Consider now $\alpha<\frac{2}{\varphi_{\max }}$. Reasoning as before, we can assume $Q(\mathbf{j}) \cap E=q\left(\mathbf{i}^{\prime}\right) \cup q\left(\mathbf{i}^{\prime \prime}\right)$. In cases $B, C$ and $D$, if $\mathbf{i}^{\prime}$ and $\mathbf{i}^{\prime \prime}$ were nearest-neighbors, with, e.g., $\varphi\left(\mathbf{i}^{\prime}\right)>\varphi\left(\mathbf{i}^{\prime \prime}\right)$, then removing $q\left(\mathbf{i}^{\prime}\right)$ would produce a negative variation; that is,

$$
\begin{aligned}
\mathcal{F}_{\alpha}^{\varphi}\left(q\left(\mathbf{i}^{\prime \prime}\right), q\right)-\mathcal{F}_{\alpha}^{\varphi}\left(q\left(\mathbf{i}^{\prime}\right) \cup q\left(\mathbf{i}^{\prime \prime}\right), q\right) & \leq 2-\alpha \varphi\left(\mathbf{i}^{\prime}\right)<2-\alpha\left(\frac{4}{\alpha}-\varphi_{\max }\right) \\
& \leq-2+\alpha \varphi_{\max } .
\end{aligned}
$$

Thus, the minimal configuration is the even checkerboard. For what concerns the case $A$, since $\varphi\left(\mathbf{j}+\mathbf{e}_{\mathbf{j}}^{3}\right)>\frac{4}{\alpha}$, by (5.8), we have that

$$
\mathcal{F}_{\alpha}^{\varphi}(q(\mathbf{j}), q)<\mathcal{F}_{\alpha}^{\varphi}\left(q(\mathbf{j}) \cup q\left(\mathbf{j}+\mathbf{e}_{\mathbf{j}}^{3}\right), q\right) \leq \mathcal{F}_{\alpha}^{\varphi}\left(q\left(\mathbf{j}+\mathbf{e}_{\mathbf{j}}^{1}\right) \cup q\left(\mathbf{j}+\mathbf{e}_{\mathbf{j}}^{2}\right), q\right)
$$

which again leads to the result. 

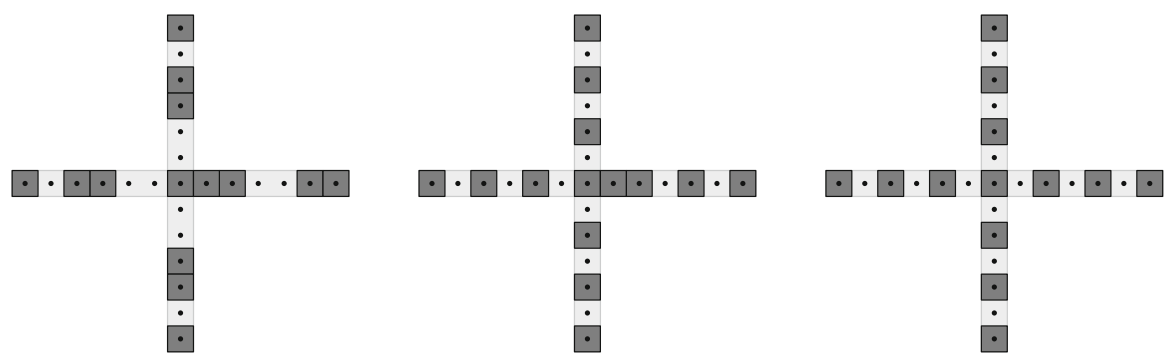

Fig. 13 Optimal configuration for $E_{\alpha, \varphi} \cap \mathcal{C}_{0}$

(c) Finally, we consider $E_{\alpha, \varphi} \cap \mathcal{C}_{0}$. Reasoning as in the case $\frac{4}{\varphi_{\max }}<\alpha<\frac{4}{\varphi_{\min }}$, we can restrict our analysis to competitors having a checkerboard structure union $q$ on the coordinate axes. A comparison between the two energy contributions on each axis yields that the variation from the odd checkerboard to the even one is less than 0 and equals 0 if and only if $\alpha \in \Lambda$. Thus, the minimal configuration is the even checkerboard (see Fig. 13). With (5.10) and the finite superadditivity of the infimum, this implies that

$$
\begin{aligned}
& \min _{E \in \mathcal{D}, E \supset q} \mathcal{F}_{\alpha}^{\varphi}(E, q) \\
& \geq \min _{E \in \mathcal{D}, E \supset q}\left(\sum_{Q(\mathbf{j}) \in \mathcal{S}_{e}\left(E_{\alpha, \varphi}\right)} \mathcal{F}_{\alpha}^{\varphi}(E \cap Q(\mathbf{j}), q)+\mathcal{F}_{\alpha}^{\varphi}\left(E \cap \mathcal{C}_{0}, q\right)\right) \\
& \geq \sum_{Q(\mathbf{j}) \in \mathcal{S}_{e}\left(E_{\alpha, \varphi}\right)} \min _{E \in \mathcal{D}, E \supset q} \mathcal{F}_{\alpha}^{\varphi}(E \cap Q(\mathbf{j}), q)+\min _{E \in \mathcal{D}, E \supset q} \mathcal{F}_{\alpha}^{\varphi}\left(E \cap \mathcal{C}_{0}, q\right) \\
& =\sum_{Q(\mathbf{j}) \in \mathcal{S}_{e}\left(E_{\alpha, \varphi}\right)} \mathcal{F}_{\alpha}^{\varphi}\left(E\left(\mathbb{Z}_{e}^{2} \cap B_{\frac{4}{\alpha}}^{\varphi}\right) \cap Q(\mathbf{j}), q\right)+\mathcal{F}_{\alpha}^{\varphi}\left(E\left(\mathbb{Z}_{e}^{2} \cap B_{\frac{4}{\alpha}}^{\varphi}\right) \cap \mathcal{C}_{0}, q\right) \\
& =\mathcal{F}_{\alpha}^{\varphi}\left(E\left(\mathbb{Z}_{e}^{2} \cap B_{\frac{4}{\alpha}}^{\varphi}\right), q\right),
\end{aligned}
$$

whence the equality follows, thus concluding the proof. Uniqueness comes from step (c).

Note that the local minimum problems studied in points (a) and (b) in the proof above might be satisfied also by the odd checkerboard if (5.8) reduces to an equality (e.g., when $\varphi=\|\cdot\|_{1}$ ). Nevertheless, for odd checkerboards the equality in (5.12) no longer holds and this implies that $E_{\alpha, \varphi}$ is the unique minimum.

Definition 28 For every $\alpha>0, \alpha \notin \Lambda^{\varphi}$, we define the nucleus of the motion given by the scheme (5.2) as the lattice set

$$
\mathcal{N}_{\alpha}^{\varphi}:=Z\left(E_{\alpha}^{1}\right)
$$

where $E_{\alpha}^{1}=\underset{E \in \mathcal{D}, E \supset q}{\operatorname{argmin}} \mathcal{F}_{\alpha}^{\varphi}(E, q)$, which is well defined by Proposition 27. 
Fig. 14 The black dots represent the lattice set $\mathbb{Z}^{2} \cap B_{\frac{4}{\alpha}}^{\varphi}$, while the set $E_{\alpha}^{1}$ is pictured in gray

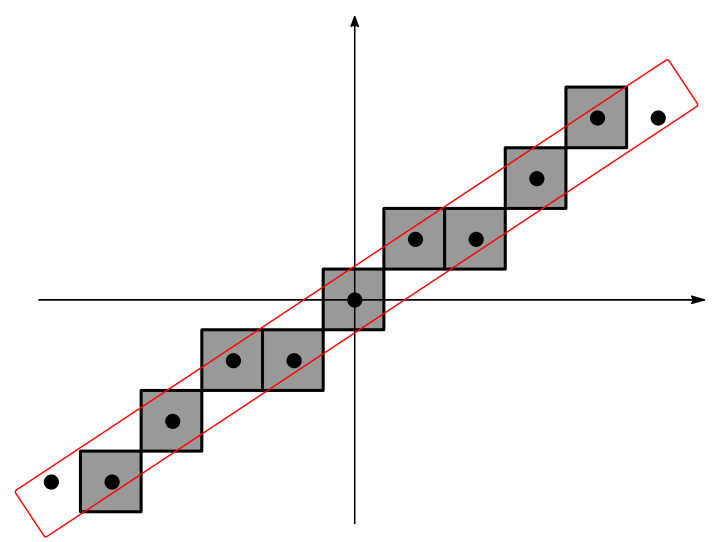

We stress that the assumption on $\varphi$ to be an absolute norm is crucial in order to obtain the previous structure result of Proposition 27. Indeed, if not fulfilled, the set $E_{\alpha}^{1}$ may not be a checkerboard as shown by the following simple example.

Example 29 (Non-checkerboard minimizers) We consider the norm

$$
\varphi(\mathbf{x})=\max \left\{\frac{\left|3 x_{1}+2 x_{2}\right|}{10},\left|3 x_{2}-2 x_{1}\right|\right\}
$$

and we assume that $\alpha \in\left(\frac{20}{13}, \frac{40}{21}\right)$. In this case, for every such $\alpha$, the set $B_{\frac{4}{\alpha}}^{\varphi}$ is a rectangle and

$$
\mathcal{J}^{\varphi, \alpha}:=B_{\frac{4}{\alpha}}^{\varphi} \cap \mathbb{Z}^{2}=\{(0,0), \pm(1,1), \pm(2,1), \pm(3,2), \pm(4,3), \pm(5,3)\}
$$

(see Fig. 14). We show that the first step of (5.2) $E_{\alpha}^{1}$ is not a checkerboard set. First note that the points $(0,0)$ and $\pm(3,2)$ are isolated in $\mathcal{J}^{\varphi, \alpha}$, so their contribution is $-4+\alpha \varphi(\mathbf{i})$ which is always negative, thus $Z\left(E_{\alpha}^{1}\right)$ contains these points. Hence, we are reduced to study the minimal configurations of the pairs of nearest-neighbors $\{(1,1),(2,1)\}$ and $\{(4,3),(5,3)\}$ :

$\mathcal{F}_{\alpha}^{\varphi}(q(1,1) \cup q(2,1), q)=-6+2 \alpha<-4+\alpha=\mathcal{F}_{\alpha}^{\varphi}(q(1,1), q)=\mathcal{F}_{\alpha}^{\varphi}(q(2,1), q)$

and

$$
\begin{aligned}
\mathcal{F}_{\alpha}^{\varphi}(q(4,3), q) & =-4+\frac{9}{5} \alpha<-6+\alpha\left(\frac{9}{5}+\frac{21}{10}\right)=\mathcal{F}_{\alpha}^{\varphi}(q(4,3) \cup q(5,3), q) \\
& <-4+\alpha \frac{21}{10}=\mathcal{F}_{\alpha}^{\varphi}(q(5,3), q) .
\end{aligned}
$$

The same holds for $\{(-1,-1),(-2,-1)\}$ and $\{(-4,-3),(-5,-3)\}$, and this gives that

$$
E_{\alpha}^{1}=E\left(\mathcal{J}^{\varphi, \alpha} \backslash\{ \pm(5,3)\}\right)
$$


which is not a checkerboard (see Fig. 14).

We conclude noting that if we renounce to the monotonicity constraint $E \supset q$, the minimization problem above may admit, for suitable values of $\alpha$, also a checkerboard solution $E_{\alpha}^{1}$ of odd parity. In order not to distract the reader's attention from the monotone case, we prefer to postpone this generalization of Proposition 27 to Sect. 6.2 (see Proposition 48).

\subsection{The Structure Result for Non-trivial Initial Datum}

Proposition 27 shows that the first step $E_{\alpha}^{1}$ of discrete scheme (5.2) is a checkerboard set and that $Z\left(E_{\alpha}^{1}\right)$ is a $\mathbb{Z}_{e}^{2}$-convex set (see Definition 3 ). Our aim now is to prove that an analogous structure result can be obtained for minimizers of the energy $\mathcal{F}_{\alpha}^{\varphi}(\cdot, E)$, where $\varphi$ is a symmetric absolute normalized norm (see Sect. 5.2), also for a general $E \in \mathcal{A}_{\text {conv }}$ fulfilling suitable assumptions (see (5.13)), and then to iteratively apply it to $E=E_{\alpha}^{k-1}$ for $k \geq 1$. The proof of this stability result will rely on a localization argument only reminiscent of that used in the proof of Proposition 27. Indeed, we have to face a technical issue: since the dissipation term $D^{\varphi}(\cdot, E)$ does not satisfy a submodularity inequality analogous to $(5.8)$, the $2 \times 2$-square covering no longer works. We will then define suitable coverings "outside" every discrete edge (see Definition 7 ) of $E$ which mimic the $2 \times 2$-square covering and then match them altogether. For this, we need the following "convexity" conditions:

(i) on the norm, we assume that

(H3) $\varphi(h, h+1)-\varphi(h, h) \geq \frac{1}{2}, \quad$ for every $h \in \mathbb{N}$;

(ii) on the structure of $\partial^{\text {eff }} E$, we require that

$\theta\left(\boldsymbol{v}\left(\ell^{\prime}\right), \boldsymbol{v}(\ell)\right)<0$ for every $\ell, \ell^{\prime} \in \mathcal{E}(E)$ such that $\ell$ precedes (clockwise) $\ell^{\prime}$,

where $\theta$ is introduced in Definition 1.

The $\ell^{p}$-norms, $1 \leq p \leq \infty$, are a class of norms complying with (H1)-(H3). We also note that assumption (H3) will play a role only in Step 5 of the proof of Proposition 30 .

In order to avoid some (interesting) pathological phenomena (as a one-dimensional motion, see Example 37), we assume non-degeneracy conditions on the sets $E$ and on the minimizer of $\mathcal{F}_{\alpha}^{\varphi}(\cdot, E)$; namely, (H2) and (2.3). Finally, to simplify the exposition, we assume that

$E$ is symmetric with respect to the axes and the lines $x_{2}= \pm x_{1}$.

We now state the main result of this section.

Proposition 30 Let $\varphi$ be a symmetric absolute normalized norm complying with (H3), and let $\alpha>0$ be such that $\alpha \notin \Lambda^{\varphi}$. Let $E \in \mathcal{A}_{\text {conv }}^{e}$ be a set satisfying (2.3), (5.13) and (5.14). Then, there exists a unique solution of the minimization problem 

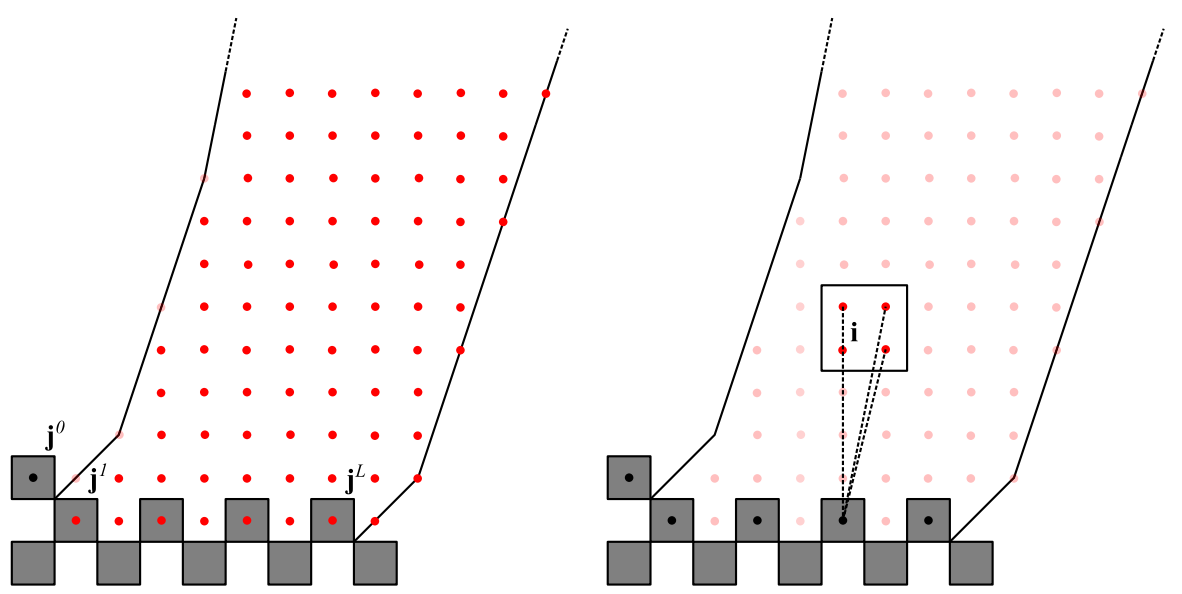

Fig. 15 In red an example of $A(\ell)$ for $\ell$ as in (ii) of Remark 8. On the left, lighter dots are outside $A(\ell)$. On the right, the projection of the centers of a $2 \times 2$-square on a common point of $\ell$

$$
E_{\alpha}=\underset{E^{\prime} \supset E E^{\prime} \in \mathcal{D}}{\operatorname{argmin}} \mathcal{F}_{\alpha}^{\varphi}\left(E^{\prime}, E\right)
$$

and it satisfies

$$
Z\left(E_{\alpha}\right)=\left\{\mathbf{i} \in \mathbb{Z}_{e}^{2}: d^{\varphi}(\mathbf{i}, E)<\frac{4}{\alpha}\right\}
$$

In particular, $E_{\alpha} \in \mathcal{A}_{\text {conv }}^{e}$.

Before entering in the details of the proof, we premise some remarks.

Remark 31 (Projection of a $2 \times 2$ square) Let $E$ be given as in the statement of Proposition 30 . We partition the lattice points of the region of the plane "outside" $E$ into sets $A(\ell)$ according to the discrete edge $\ell \in \mathcal{E}(E)$ they project onto. We follow the classification of discrete edges given in Remark 8 , and we start with case (ii); that is, $\ell \subset\left\{\mathbf{x} \in \mathbb{R}^{2}: x_{2}>0\right\}$ and $s(\ell) \in\left(0, \frac{1}{3}\right]$. For such edges, we define the set

$$
A(\ell):=\left\{\mathbf{i} \in \mathbb{Z}^{2}: i_{1} \geq j_{1}^{1}, i_{2} \geq j_{2}^{1}, \pi_{E}^{\varphi}(\mathbf{i}) \subset\left\{\mathbf{j}^{l}\right\}_{l=1}^{L} \text { or } \pi_{E}^{\varphi}(\mathbf{i}) \ni \mathbf{j}^{L}\right\}
$$

consisting of all the lattice points that project on $\ell \backslash\left\{\mathbf{j}^{0}\right\}$ (Fig. 15). The choice of excluding the points projecting also on $\mathbf{j}^{0}$, although arbitrary, will simplify the definition of the covering in the proof of Proposition 30; moreover, thanks to this choice, if $\ell$ and $\ell^{\prime}$ are two consecutive edges, then $A(\ell)$ and $A\left(\ell^{\prime}\right)$ are disjoint.

We can assume, up to translations and for the sake of simplicity, that $\ell:=\left\{\mathbf{j}^{l}\right\}_{l=0}^{L}=$ $\{(1,1)\} \cup\{(2 l, 0)\}_{l=1}^{L}$. From the fact that $\varphi$ is monotonic, for every $\mathbf{i} \in A(\ell)$ it holds that

$$
\pi_{E}^{\varphi}(\mathbf{i}) \ni \begin{cases}\mathbf{j}^{l} & 2 l-1 \leq i_{1} \leq 2 l+1 \text { with } 0<l<L \\ \mathbf{j}^{L} & i_{1} \geq 2 L-1 .\end{cases}
$$



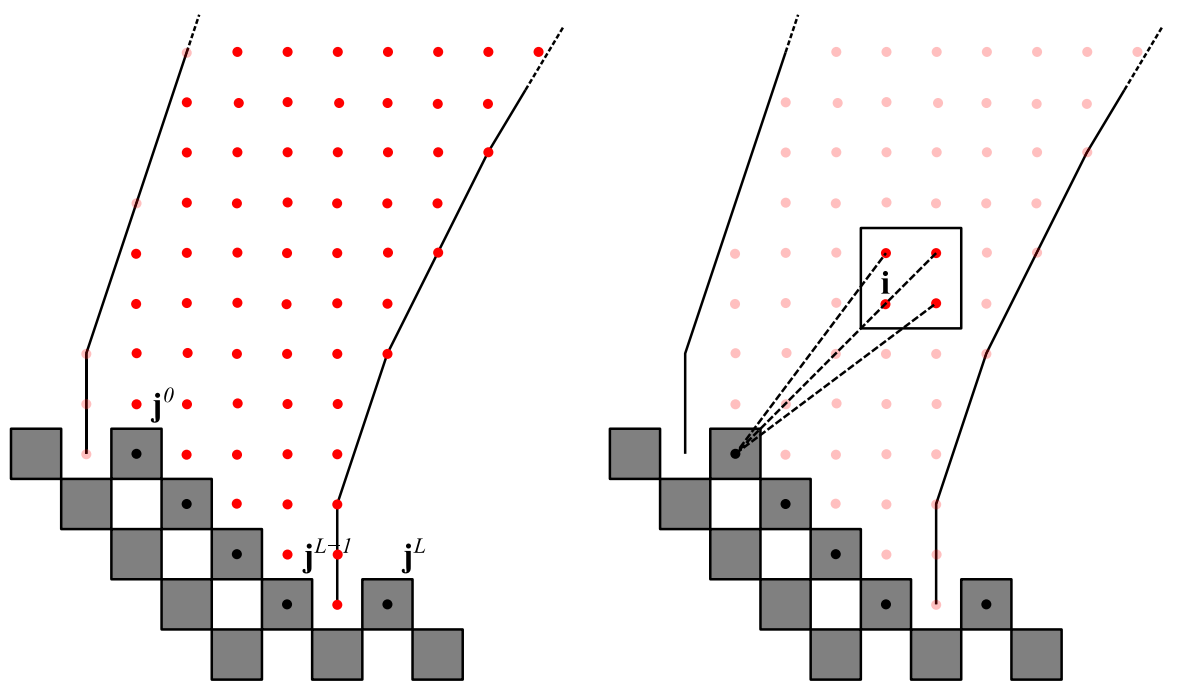

Fig. 16 In red an example of $A(\ell)$ for $\ell$ as in (iii) of Remark 8. On the left, lighter dots are outside $A(\ell)$. On the right, the projection of the centers of a $2 \times 2$-square on a common point of $\ell$

This yields that for every $\mathbf{i} \in A(\ell)$ such that $Z(Q(\mathbf{i})) \subset A(\ell)$, there holds

$$
\left(\bigcap_{\mathbf{j} \in Z(Q(\mathbf{i}))} \pi_{E}^{\varphi}(\mathbf{j})\right) \cap\left(\ell \backslash\left\{\mathbf{j}^{0}\right\}\right) \neq \varnothing
$$

This means that the four lattice points inside $Q(\mathbf{i})$ project onto a common point of $\ell$, see Fig. 15. An analogous result holds in case (i) of Remark 8, when $s(\ell)=0$.

Now, consider $\ell \in \mathcal{E}(E)$ complying with case (iii) of Remark 8; that is, $\ell \subset\{\mathbf{x} \in$ $\left.\mathbb{R}^{2}: x_{2}>0\right\}$ and $s(\ell) \in\left(\frac{1}{3}, 1\right)$. In this case, the sets of lattice points that project on $\ell \backslash\left\{\mathbf{j}^{L}\right\}$ is defined as

$$
\begin{aligned}
& A(\ell):=\left\{\mathbf{i} \in \mathbb{Z}^{2}:\left\|\mathbf{i}-\left(j_{1}^{0}, j_{2}^{L-1}\right)\right\|_{1} \geq\left|j_{2}^{0}-j_{2}^{L-1}\right|, i_{2} \geq j_{2}^{L-1},\right. \\
& \left.\quad \pi_{E}^{\varphi}(\mathbf{i}) \subset\left\{\mathbf{j}^{l}\right\}_{l=0}^{L-1} \text { or } \pi_{E}^{\varphi}(\mathbf{i}) \ni \mathbf{j}^{L-1}\right\},
\end{aligned}
$$

see Fig. 16. For simplicity, we can assume, up to translations, that $\ell=\left\{\mathbf{j}^{l}\right\}_{l=0}^{L}=$ $\{(l,-l)\}_{l=0}^{L-1} \cup\{(L+1,-L+1)\}$. From the symmetry assumption (H1) there holds

$$
\pi_{E}^{\varphi}(\mathbf{i}) \ni \begin{cases}\mathbf{j}^{0} & i_{1}-i_{2} \leq 1 \\ \mathbf{j}^{l} & 2 l-1 \leq i_{1}-i_{2} \leq 2 l+1 \text { with } 0<l<L\end{cases}
$$

This can be seen by characterizing the projection of points $\mathbf{i} \in A(\ell)$ of coordinates $\mathbf{i}=(h, h)$ and $(h+1, h)$ with $h \in \mathbb{N}$, since the other cases reduce to this situation from the translation invariance of the distance. Thus, assume by contradiction that there exist $h$ and $0<l<L$ such that $\varphi\left(\mathbf{i}-\mathbf{j}^{l}\right)=\varphi(h-l, h+l)<\varphi(h, h)=\varphi\left(\mathbf{i}-\mathbf{j}^{0}\right)$. We 

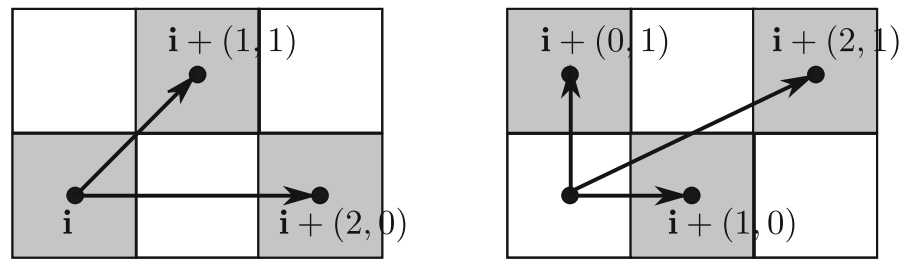

Fig. 17 The triples of points involved in (5.21)

reduce to $l \leq h$ from the fact that $\varphi$ is monotonic. Then, by (H1) and convexity we get

$$
\varphi(h, h) \leq \frac{1}{2} \varphi(h+l, h-l)+\frac{1}{2} \varphi(h-l, h+l)=\varphi(h-l, h+l),
$$

leading to a contradiction. As for the case $\mathbf{i}=(h+1, h)$, assuming that $\varphi\left(\mathbf{i}-\mathbf{j}^{l}\right)<$ $\varphi\left(\mathbf{i}-\mathbf{j}^{0}\right)$ again by $(\mathrm{H} 1)$ and convexity we get

$$
\begin{aligned}
\varphi(h+1, h) \leq & \frac{h+1}{2 h+1} \varphi(h+1-l, h+l) \\
& +\frac{h}{2 h+1} \varphi(h+l, h+1-l)=\varphi(h+1-l, h+l)
\end{aligned}
$$

and we obtain a contradiction. Hence, for every $\mathbf{i} \in A(\ell)$ such that $Z(Q(\mathbf{i})) \subset A(\ell)$ there holds

$$
\left(\bigcap_{\mathbf{j} \in Z(Q(\mathbf{i}))} \pi_{E}^{\varphi}(\mathbf{j})\right) \cap\left(\ell \backslash\left\{\mathbf{j}^{L}\right\}\right) \neq \emptyset
$$

again, as for (5.18), (5.20) means that the lattice points inside $Q(i)$ project onto a common point of $\ell$, see Fig. 16. An analog of (5.20) holds in the case (iv) of Remark 8 .

Remark 32 In order to compare the energies of checkerboard configurations with different parities inside certain rectangular tiles, it will be useful to establish some inequalities involving the dissipation term.

Consider $E$ as in the statement of Proposition 30 and $\ell \in \mathcal{E}(E)$ such that $\ell \subset\{\mathbf{x} \in$ $\left.\mathbb{R}^{2}: x_{2}>0\right\}$ and $s(\ell) \in[0,1]$. For the sake of simplicity, we can assume (up to a translation) that $\mathbf{j}^{L}=(0,0)$ where $\ell=\left\{\mathbf{j}^{l}\right\}_{l=0}^{L}$. If $s(\ell) \in\left[0, \frac{1}{3}\right]$, for every $\mathbf{i} \in A(\ell)$ with $i_{1} \in 2 \mathbb{Z}$ such that (5.18) holds, from (5.8) and the properties of $\varphi$ one can infer (see Fig. 17) the inequality

$$
\begin{aligned}
& d^{\varphi}(\mathbf{i}, E)+d^{\varphi}(\mathbf{i}+(1,1), E)+d^{\varphi}(\mathbf{i}+(2,0), E) \\
& \quad \leq d^{\varphi}(\mathbf{i}+(0,1), E)+d^{\varphi}(\mathbf{i}+(1,0), E)+d^{\varphi}(\mathbf{i}+(2,1), E) .
\end{aligned}
$$

The same inequality holds if $s(\ell) \in\left(\frac{1}{3}, 1\right]$, for every $\mathbf{i} \in A(\ell)$ with $\mathbf{i} \in \mathbb{Z}_{e}^{2}$ such that (5.20) holds. 
Indeed, (5.18) and (5.20) ensure the existence of some $\mathbf{j}^{\prime} \in \ell$ such that $d^{\varphi}(\mathbf{j}, E)=$ $\varphi\left(\mathbf{j}-\mathbf{j}^{\prime}\right)$ for every $\mathbf{j} \in Z(Q(\mathbf{i}))$. Hence, (5.8) reads

$$
d^{\varphi}(\mathbf{i}, E)+d^{\varphi}(\mathbf{i}+(1,1), E) \leq d^{\varphi}(\mathbf{i}+(0,1), E)+d^{\varphi}(\mathbf{i}+(1,0), E) .
$$

Now, from the fact that $i_{2} \geq j_{2}^{\prime}$ (see Remark 31) and the monotonicity of the norm $\varphi$, we have $\varphi\left(\mathbf{i}+(2,0)-\mathbf{j}^{\prime}\right) \leq \varphi\left(\mathbf{i}+(2,1)-\mathbf{j}^{\prime}\right)$, whence we get

$$
d^{\varphi}(\mathbf{i}+(2,0), E) \leq d^{\varphi}(\mathbf{i}+(2,1), E)
$$

Inequality (5.21) then follows by adding term by term (5.22) and (5.23).

Remark 33 As a last preparatory remark to the proof of Proposition 30, we analyze and motivate assumption (5.13) on the sets that intervene in minimization problem (5.15). Assumption (5.13) ensures that for every discrete edge, there are infinitely many $2 \times 2$-squares whose centers project onto it. This property is crucial to define a well-posed covering argument (see Sect. 5.5). Specifically, let $E$ be as in the statement of Proposition 30 and $\ell \in \mathcal{E}(E)$ be such that $\ell \subset\left\{\mathbf{x} \in \mathbb{R}^{2}: x_{2}>0\right\}$ and

$$
s(\ell) \in[0,1] .
$$

We claim that for every such $\ell$ the following property holds:

$$
\text { for every } h \in \mathbb{N} \text { there exists } \mathbf{i} \in A(\ell) \cap(\mathbb{Z} \times\{h\}) \cap \mathbb{Z}_{e}^{2}: Z(Q(\mathbf{i})) \subset A(\ell),
$$

where we have set $\ell=\left\{\mathbf{j}^{l}\right\}_{l=0}^{L}$ and $\mathbf{j}^{L}=(0,0)$ for simplicity. This claim is proved inductively (on parameter labeling clockwise consecutive discrete edges) by showing that for any triple of consecutive edges of $E$, say $\ell^{-}, \ell, \ell^{+}$with $\ell^{-}$satisfying (5.25), we can find a point $\mathbf{i} \in(\mathbb{Z} \times\{h\}) \cap \mathbb{Z}_{e}^{2}$ for which, thanks to (5.13) and the translation invariance of the distance, there holds $d^{\varphi}(\mathbf{j}, \ell) \leq d^{\varphi}\left(\mathbf{j}, \ell^{-} \cup \ell^{+}\right)$for every $\mathbf{j} \in Z(Q(\mathbf{i}))$ and every $h \geq 0$.

Let $\ell_{0}=\left\{\mathbf{j}_{0}^{l}\right\}_{l=0}^{L_{0}}$ be the first (clockwise-ordered) edge such that $s(\ell) \geq 0$ and set

$$
\ell_{0}^{\prime}:= \begin{cases}\ell_{0} & \text { if } \boldsymbol{v}\left(\ell_{0}\right)=(0,1) \\ \left\{\mathbf{j}_{0}^{0}\right\} & \text { otherwise }\end{cases}
$$

It is straightforward that (5.25) is satisfied for $\ell=\ell_{0}^{\prime}$ (reasoning as in Remark 31) where we have set

$$
A\left(\mathbf{j}_{0}^{0}\right)=\left\{\mathbf{i} \in \mathbb{Z}^{2}: \pi_{E}^{\varphi}(\mathbf{i}) \ni \mathbf{j}_{0}^{0}\right\}
$$

Consider $\ell, \ell^{-}, \ell^{+} \in \mathcal{E}(E)$ satisfying (5.24), with $\ell^{-}$preceding $\ell, \ell$ preceding $\ell^{+}$. Write $\ell^{-}=\left\{\mathbf{j}^{-, l}\right\}_{l=0}^{L^{-}}$and $\ell^{+}=\left\{\mathbf{j}^{+, l}\right\}_{l=0}^{L^{+}}$. We point out that if $\ell^{-}$coincide with $\ell_{0}=\left\{\mathbf{j}_{0}^{0}\right\}$, then $\ell=\ell_{0}$. 


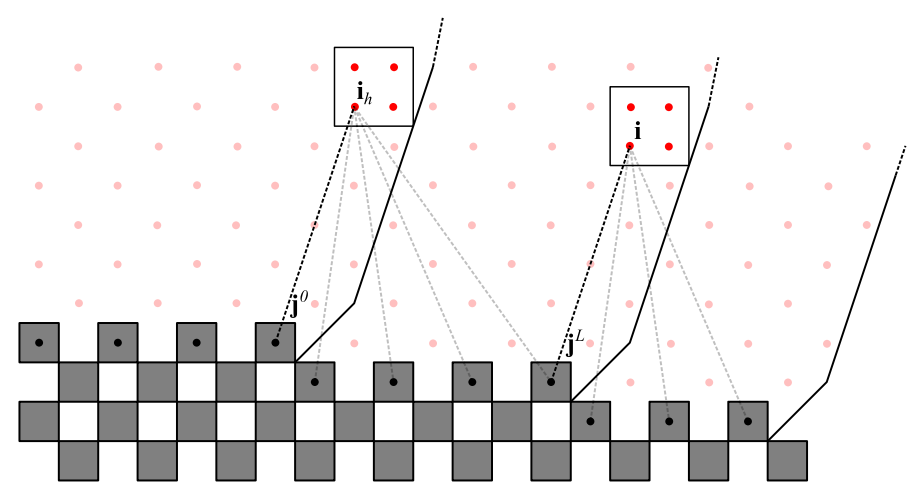

Fig. 18 An example of the situation described in Remark 33 in the case $s(\ell) \leq \frac{1}{3}$. The lighter dots represent the points of $\mathbb{Z}_{e}^{2}$ inside $A\left(\ell^{-}\right), A(\ell)$ and $A\left(\ell^{+}\right)$

Assume that $\ell^{-}$satisfies (5.25). Consider first the case $s(\ell) \leq \frac{1}{3}$ (see Fig. 18). For any fixed $h \in \mathbb{N}$, we set $\mathbf{i}_{h}=\operatorname{argmax}\left\{i_{1}: \mathbf{i} \in \mathbb{Z}_{e}^{2}, Z(Q(\mathbf{i})) \subset A\left(\ell^{-}\right), i_{2}=h+1\right\}$, which is well defined since we have assumed that $\ell^{-}$satisfies (5.25). By Remark 31 and by definition of $A\left(\ell^{-}\right)$(5.17), there holds

$$
d^{\varphi}(\mathbf{j}, E)=\varphi\left(\mathbf{j}-\mathbf{j}_{0}\right)<d^{\varphi}(\mathbf{j}, \ell) \text { for every } \mathbf{j} \in Z\left(Q\left(\mathbf{i}_{h}\right)\right) .
$$

Set $\mathbf{i}:=\mathbf{i}_{h}+(2 L-1,-1)$ and note that $\mathbf{i} \in \mathbb{Z}_{e}^{2}$ and $i_{2}=h$. Note also that the definition of $\mathbf{i}_{h}$ yields $Z(Q(\mathbf{i})) \cap A\left(\ell^{-}\right)=\emptyset$. Then, by (5.27) and the translation invariance of the distance, since $\mathbf{j}_{0}+(2 L-1,-1)=\mathbf{j}_{L}$, we get

$$
d^{\varphi}(\mathbf{j}, \ell)=\varphi\left(\mathbf{j}-\mathbf{j}_{L}\right)<d^{\varphi}(\mathbf{j}, \ell+(2 L-1,-1)), \quad \text { for every } \mathbf{j} \in Z(Q(\mathbf{i})) .
$$

Now, (5.13) yields $d^{\varphi}(\mathbf{j}, \ell+(2 L-1,-1)) \leq d^{\varphi}\left(\mathbf{j}, \ell^{+}\right)$. Indeed, if $s\left(\ell^{+}\right) \leq \frac{1}{3}$, then $L^{+} \leq L$ by (5.13); thus, $\ell^{+} \subset \ell+(2 L-1,-1)$. If instead $s\left(\ell^{+}\right)>\frac{1}{3}$, then by the monotonicity of $\varphi$ we get $d^{\varphi}\left(\mathbf{j}, \ell^{+}\right) \geq \varphi\left(\mathbf{j}-\mathbf{j}^{L}\right)$.

Now, consider the case $s(\ell)>\frac{1}{3}$ (Fig. 19). For any fixed $h \in \mathbb{N}$, we set

$$
\mathbf{i}_{h}=\operatorname{argmax}\left\{i_{1}: \mathbf{i} \in \mathbb{Z}_{e}^{2}, Z(Q(\mathbf{i})) \subset A\left(\ell^{-}\right), i_{2}=h+L-1\right\},
$$

which is well defined as above. By Remark 31 and by definition of $A\left(\ell^{-}\right)$(5.19), there holds

$$
d^{\varphi}(\mathbf{j}, E)=\varphi\left(\mathbf{j}-\mathbf{j}^{\prime}\right) \leq \varphi\left(\mathbf{j}-\mathbf{j}^{0}\right)=d^{\varphi}(\mathbf{j}, \ell) \quad \text { for every } \mathbf{j} \in Z\left(Q\left(\mathbf{i}_{h}\right)\right)
$$

for some $\mathbf{j}^{\prime} \in\left\{j^{-, l}\right\}_{l=0}^{L^{-}-1}$. Again, set $\mathbf{i}:=\mathbf{i}_{h}+(L-1,-L+1)$ and note that $\mathbf{i} \in \mathbb{Z}_{e}^{2}$ and $i_{2}=h$. Reasoning as above, we have $Z(Q(\mathbf{i})) \cap A\left(\ell^{-}\right)=\emptyset$. By (5.28), we have the translation invariance of the distance and since $\mathbf{j}^{\prime \prime}:=\mathbf{j}^{\prime}+\left(L^{-}+1,-L^{-}+1\right) \in$ 


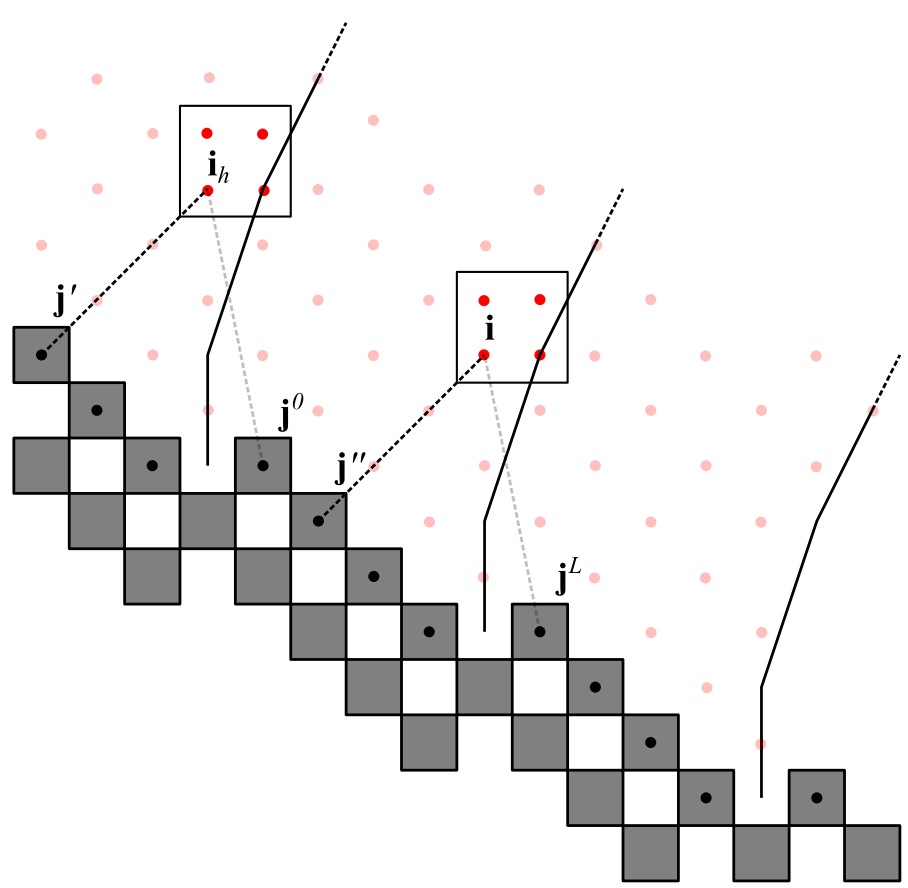

Fig. 19 An example of the situation described in Remark 33 in the case $s(\ell)<\frac{1}{3}$. The lighter dots represent those points of $\mathbb{Z}_{e}^{2}$ lying inside $A\left(\ell^{-}\right), A(\ell)$ and $A\left(\ell^{+}\right)$

\section{$\left\{\mathbf{j}_{l}\right\}_{l=0}^{L^{-}-1} \subset \ell$ we get}

$$
d^{\varphi}(\mathbf{j}, \ell)=\varphi\left(\mathbf{j}-\mathbf{j}^{\prime \prime}\right) \leq \varphi\left(\mathbf{j}-\mathbf{j}^{L}\right), \quad \text { for every } \mathbf{j} \in Z(Q(\mathbf{i}))
$$

From (5.13), we have $s(\ell)>\frac{1}{3}$ and $L^{+} \geq L$, thus $\varphi\left(\mathbf{j}-\mathbf{j}^{L}\right)=d^{\varphi}\left(\mathbf{j}, \ell^{+}\right)$, arguing as in Remark 31.

\subsection{Proof of Proposition 30}

We are now ready to prove the main result on the structure of the minimizer of $\mathcal{F}_{\alpha}^{\varphi}(\cdot, E)$. For the covering argument that we will introduce, the $2 \times 2$-squares are not sufficient. Therefore, we define a new class of tiles for the covering.

Definition 34 For every $\mathbf{i} \in \mathbb{Z}^{2}$, we set

$$
\begin{aligned}
& R^{\text {hor }}(\mathbf{i}):=Q(\mathbf{i}) \cup q(\mathbf{i}+(2,0)) \cup q(\mathbf{i}+(2,1)), R^{+}(\mathbf{i}):=R^{\mathrm{ver}}(\mathbf{i}) \cup R^{\text {hor }}(\mathbf{i}), \\
& R^{\operatorname{ver}}(\mathbf{i}):=Q(\mathbf{i}) \cup q(\mathbf{i}+(0,2)) \cup q(\mathbf{i}+(1,2)), R^{-}(\mathbf{i}):=R^{\mathrm{ver}}(\mathbf{i}+(1,-1)) \cup R^{\text {hor }}(\mathbf{i}),
\end{aligned}
$$

where $Q(\mathbf{i})$ is defined as in (5.6) (see Fig. 20). 

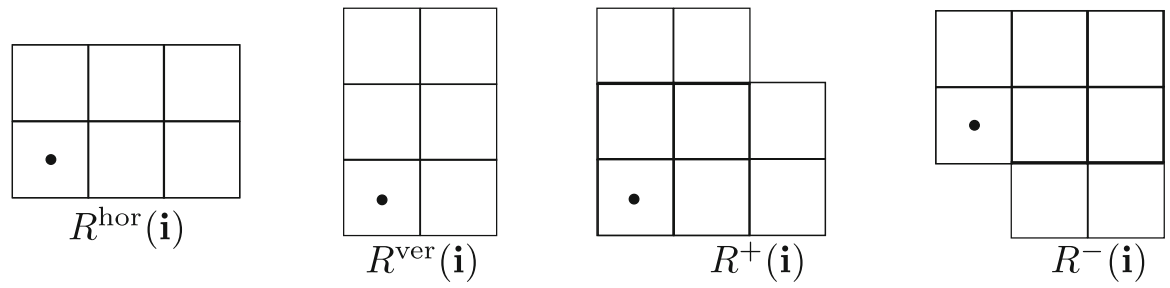

Fig. 20 The different tiles of the covering

For every discrete edge $\ell \in \mathcal{E}(E)$, we will define a covering of the region outside $E$ projecting onto $\ell$. We warn the reader that the choice of the tiles will depend both on the slope $s(\ell)$ and the neighboring edges. Heuristically, where the discrete projection $\pi_{E}^{\varphi}$ behaves as in the case of the distance from a point, we will still use the tiles $Q(\mathbf{i})$, as in the proof of Proposition 27. In order to match the coverings of the regions projecting onto adjacent edges, we will need tiles $R^{\text {hor }}$ (i) and $R^{\text {ver }}$ (i) (see Steps 2 and 3 of the proof), in which the even checkerboard is the minimizer by virtue of Remark 32. Moreover, we will take into account that the effective boundary $\partial^{\text {eff }} E$ may present some irregularities due to the discrete nature of the problem (see Steps 4 and 5). In that case, where needed, we will use the "siding tiles" $R^{+}(\mathbf{i})$ and $R^{-}(\mathbf{i})$ which are compatible with the rest of the covering and still favor the even configurations in the local minimum problems therein.

Proof of Proposition 30 According to the discussion in Remark 8, we reduce the description of the covering corresponding to the discrete edges of $E$ contained in $\left\{\mathbf{x} \in \mathbb{R}^{2}: x_{2} \geq 0\right\}$ complying with

$$
0 \leq s(\ell) \leq 1
$$

as the covering for the remaining edges can be obtained symmetrically. We divide the proof into several steps.

Step 1: ordering of the discrete edges. We label in clockwise order the set of discrete edges of $E$; namely, $\left\{\ell_{m}\right\}_{m=1}^{m_{1}} \subset \mathcal{E}(E)$. For our convenience, writing $\ell_{1}=$ $\left\{\mathbf{j}^{l}\right\}_{l=0}^{L}$, with a slight abuse of notation, in the case that $s\left(\ell_{1}\right)=0$ we write (without relabeling) $\ell_{1}=\left\{\mathbf{j}_{1}^{l}\right\}_{l=\left\lfloor\frac{L}{2}\right\rfloor}^{L}$. If $s\left(\ell_{1}\right)>0$, we set $\ell_{0}:=\left\{\mathbf{j}_{1}^{0}\right\}$. Now, we set

$$
m_{0}:=\max \left\{0 \leq m \leq M: s\left(\ell_{m}\right) \leq \frac{1}{3}\right\}
$$

Step 2: covering of the region outside $E$ projecting onto $\ell_{m}$ with $0<m<m_{0}$. We set $\ell_{m}:=\left\{\mathbf{j}^{l}\right\}_{l=0}^{L}$ assuming, without loss of generality, that $\mathbf{j}^{L}=(0,0)$. We also define

$$
\begin{aligned}
& a_{h}:=\min \left\{h^{\prime} \in 2 \mathbb{Z}:\left(h^{\prime}, 2 h+1\right) \in A\left(\ell_{m}\right)\right\}, \\
& b_{h}:=\max \left\{h^{\prime} \in 2 \mathbb{Z}:\left(h^{\prime}+1,2 h\right) \in A\left(\ell_{m}\right)\right\},
\end{aligned}
$$




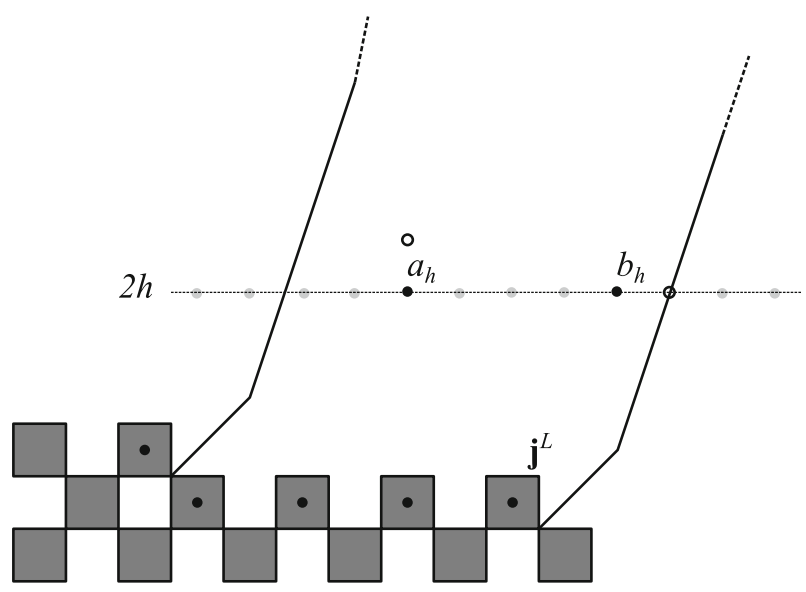

Fig. 21 The point $\left(a_{h}, 2 h+1\right) \in A(\ell)$ (black circle on the left). The point $\left(b_{h}+1,2 h\right) \in A\left(\ell_{m+1}\right)($ black circle on the right). The gray points represent $\mathbb{Z} \times\{2 h\}$
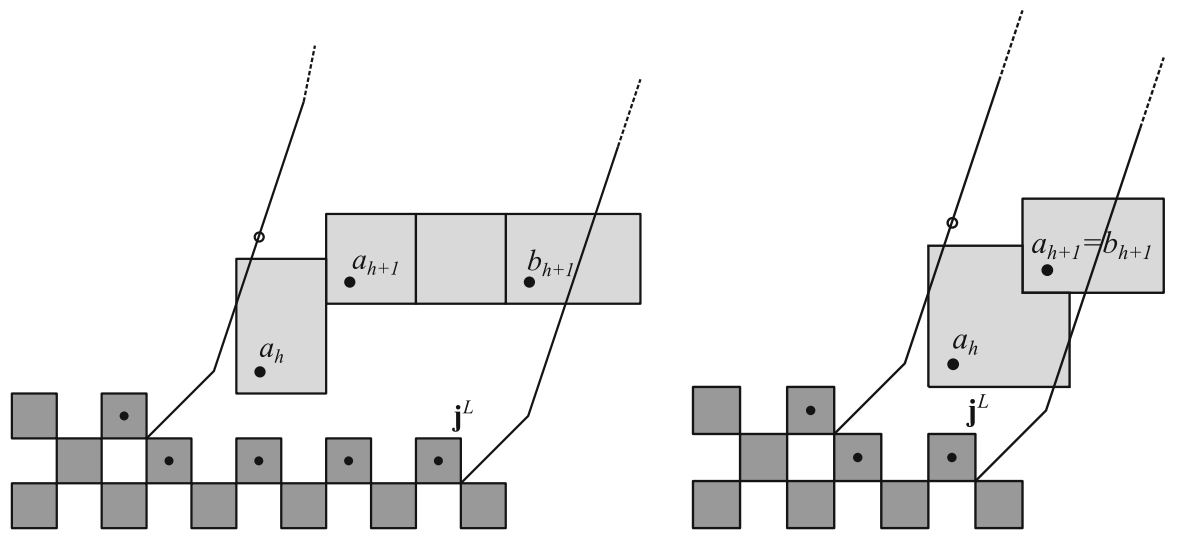

Fig. 22 Examples of coverings defined in (5.32). The black circle represents the point $\left(a_{h-1}, 2 h+3\right)$

for every $h \in \mathbb{N}$, where $A(\ell)$ is defined in (5.17) (see Fig. 21). In the case $m=1$ and $s\left(\ell_{1}\right)=0$, the set $A\left(\ell_{1}\right)$ is still as in (5.17) with $\left\{\mathbf{j}^{l}\right\}_{l=\left\lfloor\frac{L}{2}\right\rfloor}^{L}$ in place of $\left\{\mathbf{j}^{l}\right\}_{l=1}^{L}$. Note that, by Remark 33, assumption (5.13) yields that $a_{h}$ and $b_{h}$ are well-defined for every $h \in \mathbb{N}$. We then introduce the set

$$
\mathcal{J}\left(\ell_{m}\right):=\bigcup_{h \geq 0}\left\{\left(h^{\prime}, 2 h\right): h^{\prime} \in 2 \mathbb{Z}, a_{h} \leq h^{\prime} \leq b_{h}\right\} .
$$



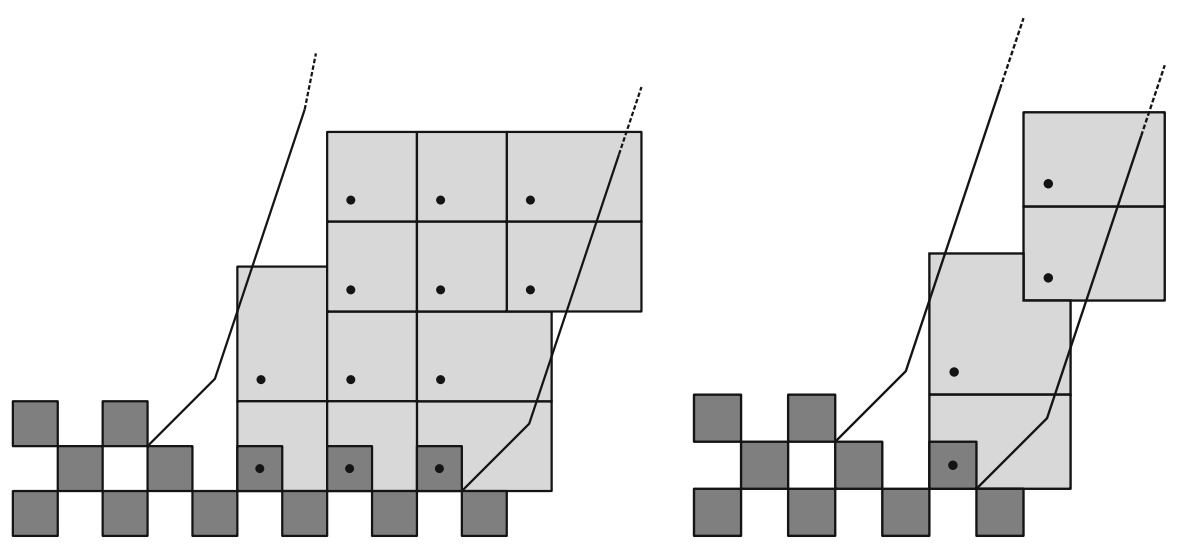

Fig. 23 Examples of whole coverings defined in formula (5.32). The black dots represent the points of $\mathcal{J}\left(\ell_{m}\right)$

Correspondingly, for every $h \in \mathbb{N}$ we define the following covering (see Fig. 22):

$$
\begin{aligned}
& C(\mathbf{i}):=\left\{\begin{array}{ll}
R^{\mathrm{ver}}(\mathbf{i}) & \mathbf{i}=\left(a_{h}, 2 h\right) \text { and }\left(a_{h}, 2 h+3\right) \notin A\left(\ell_{m}\right) \\
Q(\mathbf{i}) & \mathbf{i}=\left(a_{h}, 2 h\right) \text { and }\left(a_{h}, 2 h+3\right) \in A\left(\ell_{m}\right) \\
Q(\mathbf{i}) & i_{1} \in 2 \mathbb{Z}, a_{h}<i_{1}<b_{h} \\
R^{\text {hor }}(\mathbf{i}) & \mathbf{i}=\left(b_{h}, 2 h\right)
\end{array} \text { if } a_{h}<b_{h},\right. \\
& C(\mathbf{i}):=\left\{\begin{array}{ll}
R^{+}(\mathbf{i}), & \mathbf{i}=\left(a_{h}, 2 h\right) \text { and }\left(a_{h}, 2 h+3\right) \notin A\left(\ell_{m}\right) \\
R^{\text {hor }}(\mathbf{i}), & \mathbf{i}=\left(a_{h}, 2 h\right) \text { and }\left(a_{h}, 2 h+3\right) \in A\left(\ell_{m}\right)
\end{array}, \quad \text { if } a_{h}=b_{h},\right.
\end{aligned}
$$

(see Fig. 23 for an example of $\left.\left\{C(\mathbf{i}): \mathbf{i} \in \mathcal{J}\left(\ell_{m}\right)\right\}\right)$.

Step 3: covering of the region outside $E$ projecting onto $\ell_{m}$ with $m_{0} \leq m \leq$ $m_{1}-1$. As before, we label clockwise the set of points $\left\{\mathbf{j}^{r}\right\}_{r \geq 0}=\bigcup_{m=m_{0}+1}^{m_{1}} \ell_{m} \backslash\left\{\ell_{0}\right\}$. For every $m_{0}+1 \leq m \leq m_{1}-1$, writing $\ell_{m}=\left\{\mathbf{j}^{l}\right\}_{l=0}^{L}$ we define

$$
r_{0}:=\min \left\{r \in 2 \mathbb{Z}: \mathbf{j}^{r} \in \ell_{m}\right\} \text { and } r_{1}:=\max \left\{r \in 2 \mathbb{Z}: \mathbf{j}^{r} \in \ell_{m} \backslash\left\{\mathbf{j}^{L}\right\}\right\}
$$

Fix first $\ell_{m}$ with $m_{0}+1<m \leq m_{1}-1$ and assume, without loss of generality, that $\mathbf{j}^{r_{1}}=(0,0)$. Now, for every $h \in \mathbb{N}$ we determine the integers $a_{h}$ and $b_{h}$ as in (5.30) (see Fig. 24), which are well defined by Remark 33, where $A(\ell)$ is as in (5.19). Correspondingly, we define the sets $\mathcal{J}\left(\ell_{m}\right)$ as in (5.31) and $C(\mathbf{i})$ for every $\mathbf{i} \in \mathcal{J}\left(\ell_{m}\right)$ as in (5.32), respectively (see Figs. 25 and 26).

The covering outside the discrete edges $\ell_{m_{0}}$ and $\ell_{m_{0}+1}$ must be treated separately. Let $r_{0}, r_{1}$ be as in (5.33) with $m=m_{0}+1$. Again, we assume that $\mathbf{j}^{r_{1}}=(0,0)$, define $a_{h}, b_{h}$ as in (5.30) for every $h \in \mathbb{N}$ with $A\left(\ell_{m_{0}}\right) \cup A\left(\ell_{m_{0}+1}\right)$ in place of $A\left(\ell_{m}\right)$ and the set $\mathcal{J}\left(\ell_{m_{0}} \cup \ell_{m_{0}+1}\right)$ as in (5.31). The sets $C(\mathbf{i})$ are defined, for every $\mathbf{i} \in \mathcal{J}\left(\ell_{m_{0}} \cup \ell_{m_{0}+1}\right)$, as in (5.32) with $A\left(\ell_{m_{0}}\right) \cup A\left(\ell_{m_{0}+1}\right)$ in place of $A\left(\ell_{m}\right)$ (see Fig. 27). Note that in this case $a_{h} \neq b_{h}$ for every $h \in \mathbb{N}$. 
Fig. 24 The point

$\left(a_{h}, 2 h+1\right) \in A\left(\ell_{m}\right)$ (black circle on the left). The point $\left(b_{h}+1,2 h\right) \in A\left(\ell_{m}\right)$ (black circle on the right)
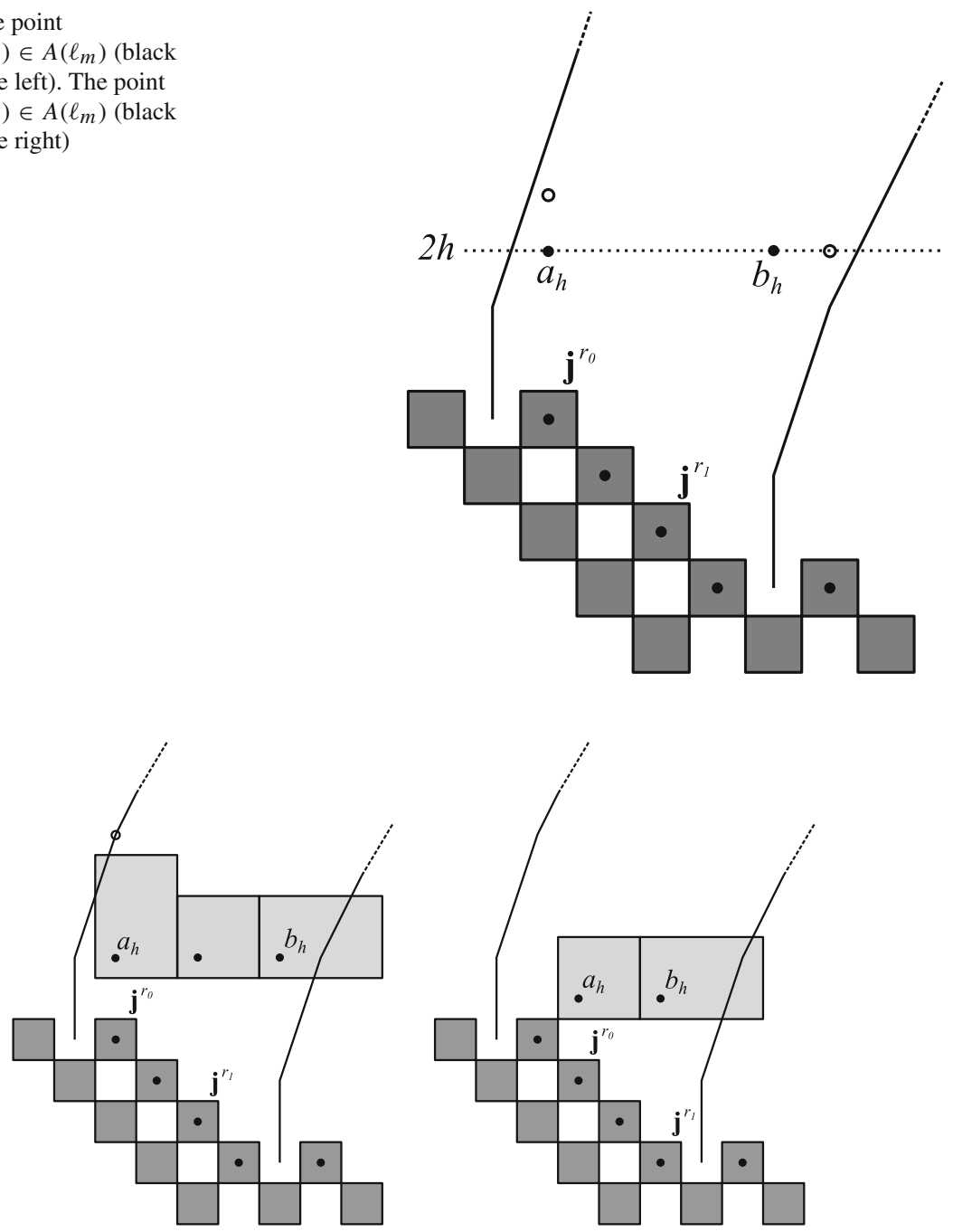

Fig. 25 Examples of coverings of the two possible parities defined in formula (5.32). The black circle represents the point $\left(a_{h}, 2 h+3\right)$

Step 4: covering of the region outside $E$ projecting onto $\ell_{m_{1}}$. We set $\ell_{m_{1}}=\left\{\mathbf{j}^{l}\right\}_{l=0}^{L}$ with $\mathbf{j}^{L}=(0,0)$. There are different possible cases depending on $\boldsymbol{v}\left(\ell_{m_{1}}\right)$ :

(i) let $m_{0}=m_{1}$; i.e., $s\left(\ell_{m}\right) \leq \frac{1}{3}$ for every $m$. We set, for every $h \in \mathbb{N}, a_{h}$ as in (5.30), $b_{h}=2 h$ and $\mathcal{J}\left(\ell_{m_{1}}\right)$ as in (5.31). Then, $C$ (i) is defined as in (5.32) for every $\mathbf{i} \in \mathcal{J}\left(\ell_{m_{1}}\right) \backslash\left\{\left(b_{h}, 2 h\right)\right\}_{h \in \mathbb{N}}$ and $C\left(\left(b_{h}, 2 h\right)\right)=Q\left(\left(b_{h}, 2 h\right)\right)$ for every $h \in \mathbb{N}$ (Fig. 28);

(ii) let $\frac{1}{3}<s\left(\ell_{m_{1}}\right)<1$ and let $r_{1}$ be defined as in (5.33) with $m=m_{1}$. Then, $a_{h}$ and $b_{h}$ are as in (5.30) for every $h \in \mathbb{N}$ with $A\left(\ell_{m_{1}}\right)$ or $A\left(\ell_{m_{1}}\right) \cup A\left(\ell_{m_{1}-1}\right)$ in place of $A\left(\ell_{m}\right)$ whether $m_{1}-1>m_{0}$ or $m_{1}-1=m_{0}$, respectively. We define $\mathcal{J}\left(\ell_{m_{1}}\right)$ as in (5.31). If $\mathbf{j}^{r_{1}}=\mathbf{j}^{L-2}, C(\mathbf{i})$ is defined as in (5.32), whereas if $\mathbf{j}^{r_{1}}=\mathbf{j}^{L-1}, C(\mathbf{i})$ is 

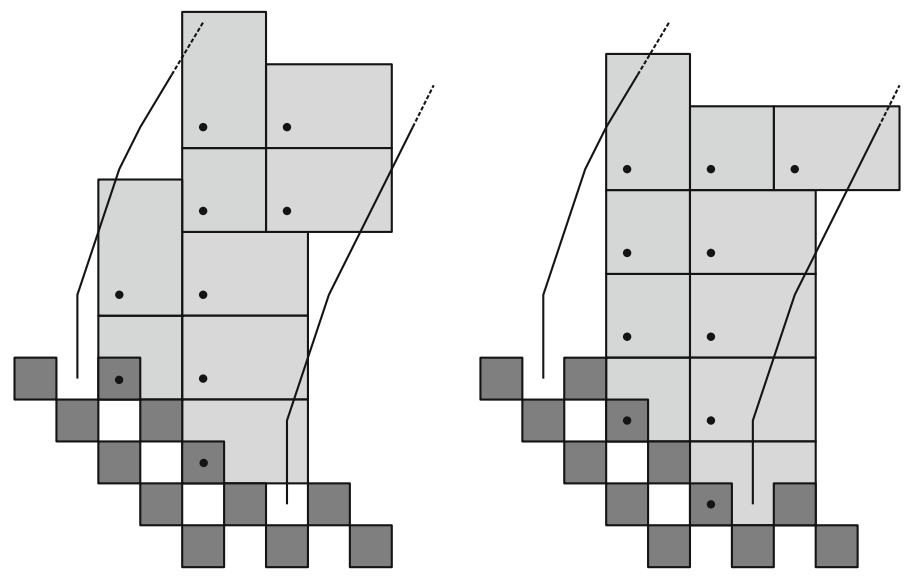

Fig. 26 Examples of whole coverings defined in formula (5.32) of two different parities. The black dots represent the points of $\mathcal{J}\left(\ell_{m}\right)$
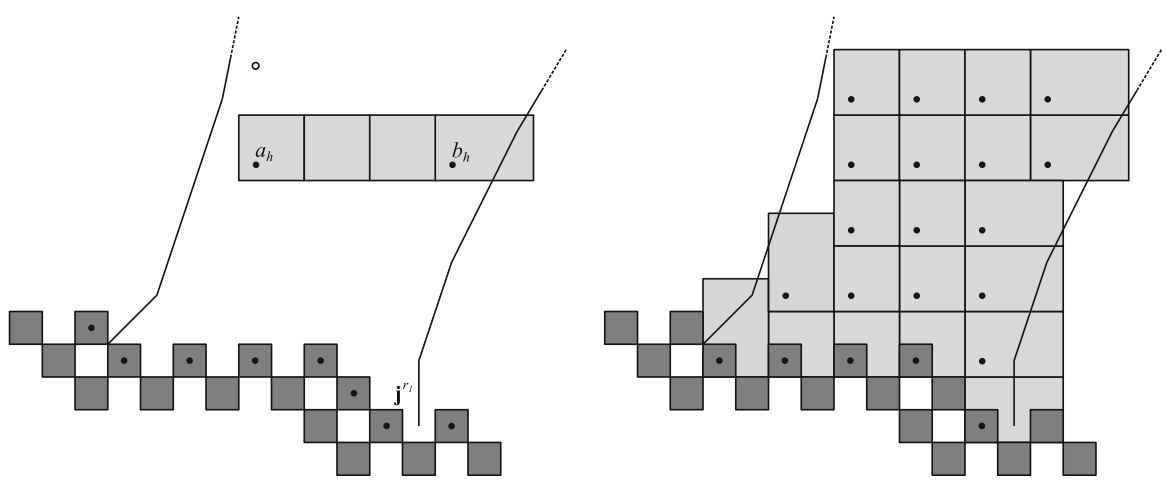

Fig. 27 On the left, an example of $a_{h}$ and $b_{h}$, and the black circle represents the point $\left(a_{h}, 2 h+3\right)$. On the right the corresponding covering, where the black dots represent the points of $\mathcal{J}\left(\ell_{m_{0}-1} \cup \ell_{m_{0}}\right)$

defined as in (5.32) for every $\mathbf{i} \in \mathcal{J}\left(\ell_{m_{1}}\right) \backslash\left\{\mathbf{j}^{r_{1}}, \mathbf{j}^{r_{1}}+(0,2)\right\}$ and

$$
C\left(\mathbf{j}^{r_{1}}\right)=\emptyset, \quad C\left(\mathbf{j}^{r_{1}}+(0,2)\right)=R^{-}\left(\mathbf{j}^{r_{1}}+(0,2)\right) .
$$

Then, setting $A\left(\mathbf{j}^{L}\right)=\left\{\mathbf{i} \in \mathbb{Z}^{2}: i_{1}, i_{2}>0, \pi_{E}^{\varphi}(\mathbf{i})=\mathbf{j}^{L}\right\}$, we introduce the integers

$$
\begin{aligned}
& a_{h}^{\prime}= \begin{cases}\min \left\{h^{\prime} \in 2 \mathbb{Z}:\left(h^{\prime}, 2 h+1\right) \in A\left(\mathbf{j}^{L}\right)\right\} & \text { if } \mathbf{j}^{r_{1}}=\mathbf{j}^{L-2} \\
\min \left\{h^{\prime} \in 2 \mathbb{Z}+1:\left(h^{\prime}, 2 h+2\right) \in A\left(\mathbf{j}^{L}\right)\right\} & \text { if } \mathbf{j}^{r_{1}}=\mathbf{j}^{L-1}\end{cases} \\
& b_{h}^{\prime}= \begin{cases}2 h & \text { if } \mathbf{j}^{r_{1}}=\mathbf{j}^{L-2} \\
2 h+1 & \text { if } \mathbf{j}^{r_{1}}=\mathbf{j}^{L-1}\end{cases}
\end{aligned}
$$




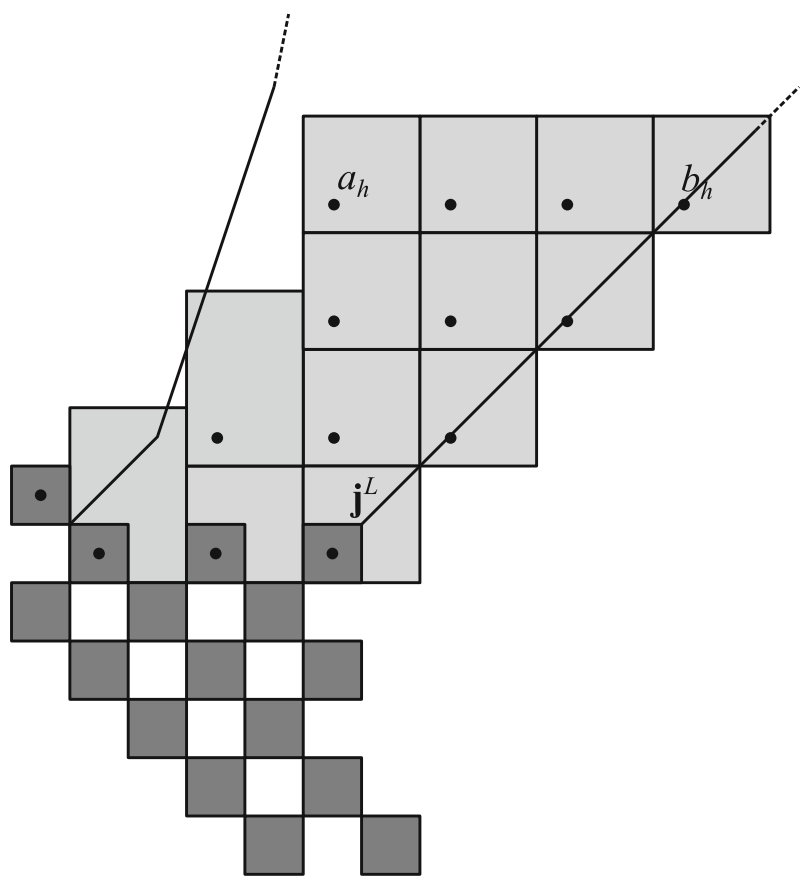

Fig. 28 Example of covering outside $\ell_{m_{1}}$ in the case (i)
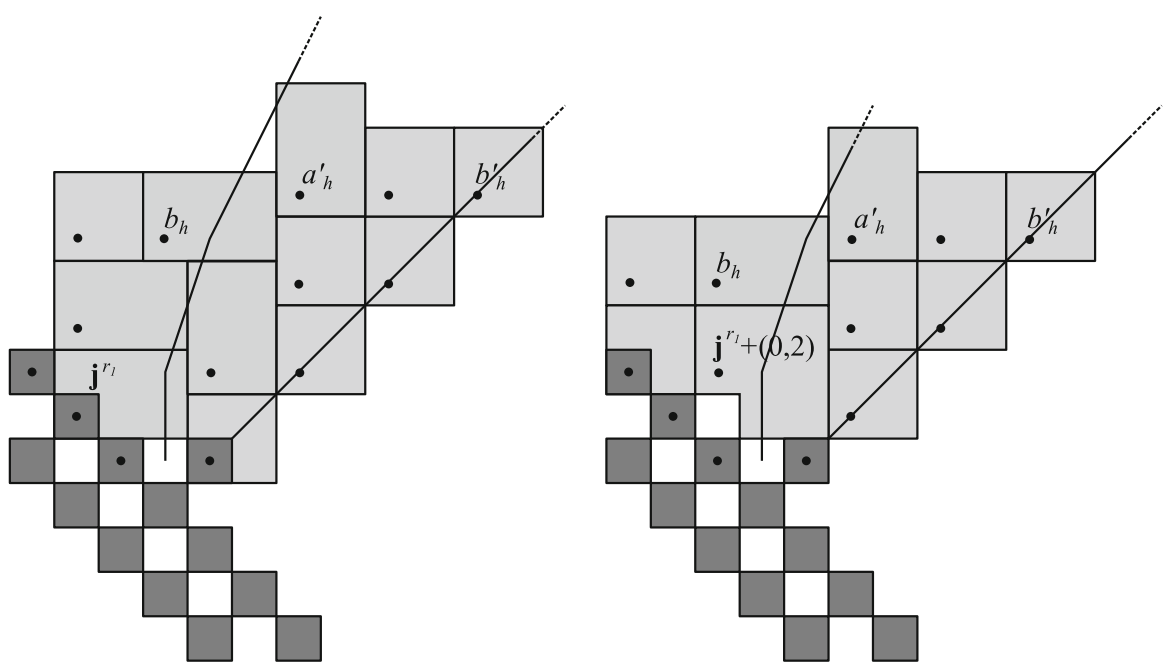

Fig. 29 The covering in the cases $\mathbf{j}^{r_{1}}=\mathbf{j}^{L-1}$ (on the left) and $\mathbf{j}^{r_{1}}=\mathbf{j}^{L}$ (on the right) 
Fig. 30 The covering considered in (iii) in the case $L$ even
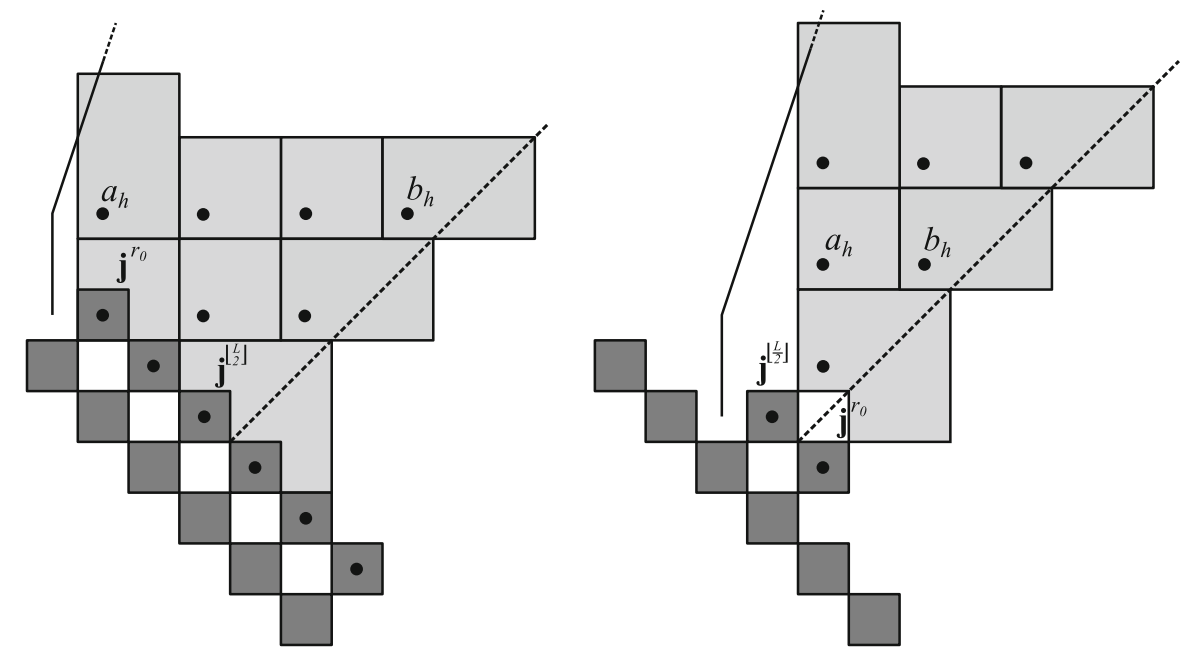

Fig. 31 The coverings defined in (iii), in the case $L$ odd, for $\mathbf{j}^{r_{0}} \neq \mathbf{j}^{L}$ (on the left) and $\mathbf{j}^{r_{0}}=\mathbf{j}^{L}$ (on the right)

Now, we define $\mathrm{J}\left(\ell_{m_{1}}^{\prime}\right)$ as in (5.31) with $a_{h}^{\prime}, b_{h}^{\prime}$ in place of $a_{h}$ and $b_{h}$, and the tile $C(\mathbf{i})$ as in (5.32) for every $\mathbf{i} \in \mathcal{J}\left(\mathbf{j}^{L}\right) \backslash\left\{\left(b_{h}^{\prime}, b_{h}^{\prime}\right)\right\}_{h \in \mathbb{N}}$, and $C\left(\left(b_{h}^{\prime}, b_{h}^{\prime}\right)\right)=Q\left(\left(b_{h}^{\prime}, b_{h}^{\prime}\right)\right)$ (see Fig. 29);

(iii) consider now the case $s\left(\ell_{m_{1}}\right)=1$. Let $r_{0}$ be defined as in (5.33) with $m=m_{1}$. Without relabeling, we set $\ell_{m_{1}}:=\left\{\mathbf{j}^{l}\right\}_{l=0}^{\left\lfloor\frac{L}{2}\right\rfloor}$ and assume $\mathbf{j}^{\left\lfloor\frac{L}{2}\right\rfloor}=(0,0)$. Here, the covering depends on the parity of $L$. If $L$ is even, $a_{h}$ is defined as in (5.30) with $m=m_{1}$ and $b_{h}=2 h$ for every $h \in \mathbb{N}$. $\mathcal{J}\left(\ell_{m_{1}}\right)$ is defined as in (5.31). Then, $C(\mathbf{i})$ is defined as in (5.32) for every $\mathbf{i} \in \mathcal{J}\left(\ell_{m_{1}}\right) \backslash\left\{\left(b_{h}, 2 h\right)\right\}_{h \in \mathbb{N}}$ and $C\left(\left(b_{h}, 2 h\right)\right)=Q\left(\left(b_{h}, 2 h\right)\right)$ (Fig. 30). 

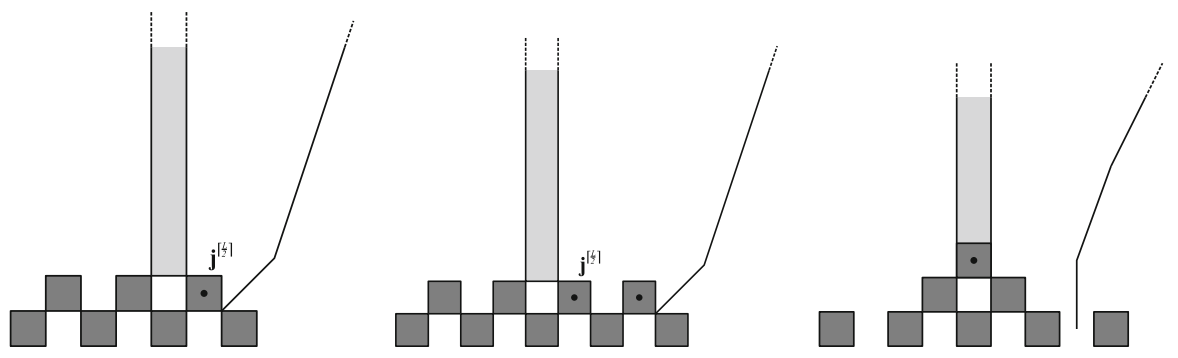

Fig. 32 The covering $\mathcal{S}_{0}$ in the cases listed in (5.34)

If $L$ is odd, analogously to what done in case (ii), for every $h \in \mathbb{N}$ we define

$$
\begin{aligned}
& a_{h}= \begin{cases}\min \left\{h^{\prime} \in 2 \mathbb{Z}:\left(h^{\prime}, 2 h+1\right) \in A\left(\ell_{m_{1}}\right)\right\} & \text { if } \mathbf{j}^{r_{0}} \neq \mathbf{j}^{L} \\
\min \left\{h^{\prime} \in 2 \mathbb{Z}+1:\left(h^{\prime}, 2 h+2\right) \in A\left(\mathbf{j}^{L}\right)\right\} & \text { if } \mathbf{j}^{r_{0}}=\mathbf{j}^{L}\end{cases} \\
& b_{h}^{\prime}= \begin{cases}2 h & \text { if } \mathbf{j}^{r_{0}} \neq \mathbf{j}^{L} \\
2 h+1 & \text { if } \mathbf{j}^{r_{1}}=\mathbf{j}^{L} .\end{cases}
\end{aligned}
$$

Then, $\mathcal{J}\left(\ell_{m_{1}}\right)$ is defined as in (5.31) and $C(\mathbf{i})$ is defined as in (5.32) for every $\mathbf{i} \in$ $\mathcal{J}\left(\ell_{m_{1}}\right) \backslash\left\{\left(b_{h}, 2 h\right)\right\}_{h \in \mathbb{N}}$ and

$$
C_{h}\left(\left(b_{h}, b_{h}\right)\right)= \begin{cases}R^{-}\left(\left(b_{h}, b_{h}\right)\right) & \text { if } h=0 \\ R\left(\left(b_{h}, b_{h}\right)\right) & \text { if } h>0\end{cases}
$$

see Fig. 31.

Step 5: covering of the region outside $E$ projecting onto $\ell_{0}$. We define the set

$$
\mathcal{S}_{0}= \begin{cases}E\left(\left\{\mathbf{i} \in \mathbb{Z}^{2}: i_{1}=j_{1}^{\left\lceil\frac{L}{2}\right\rceil}-1, i_{2} \geq 1\right\}\right) & \text { if } s\left(\ell_{1}\right)=0, \\ \emptyset & \text { if } 0<s\left(\ell_{1}\right)<\frac{1}{3}, \\ E\left(\left\{\mathbf{i} \in \mathbb{Z}^{2}: i_{1}=j_{1}^{0}, i_{2} \geq 0\right\}\right) & \text { if } \frac{1}{3}<s\left(\ell_{1}\right) \leq 1 .\end{cases}
$$

(see Fig. 32).

If $\ell$ is such that $0<s(\ell)<\frac{1}{3}$, we define $\mathcal{J}\left(\ell_{0}\right)=\{(0,2 h)\}_{h \in \mathbb{N}}$ and for every $h \in \mathbb{N}$

$$
b_{h}=\max \left\{h^{\prime} \in 2 \mathbb{Z} \mid\left(h^{\prime}+1,2 h\right) \in A\left(\mathbf{j}^{0}\right)\right\},
$$

where $A\left(\ell_{0}\right)$ is as in (5.26). Then, for every $\mathbf{i} \in \mathcal{J}\left(\ell_{0}\right)$ we choose the tile

$$
C(\mathbf{i})=\bigcup\left\{q((k, 2 h)) \cup q((k, 2 h+1)): k \in \mathbb{Z},-b_{h}-2 \leq k \leq b_{h}+2, i_{2}=2 h\right\},
$$




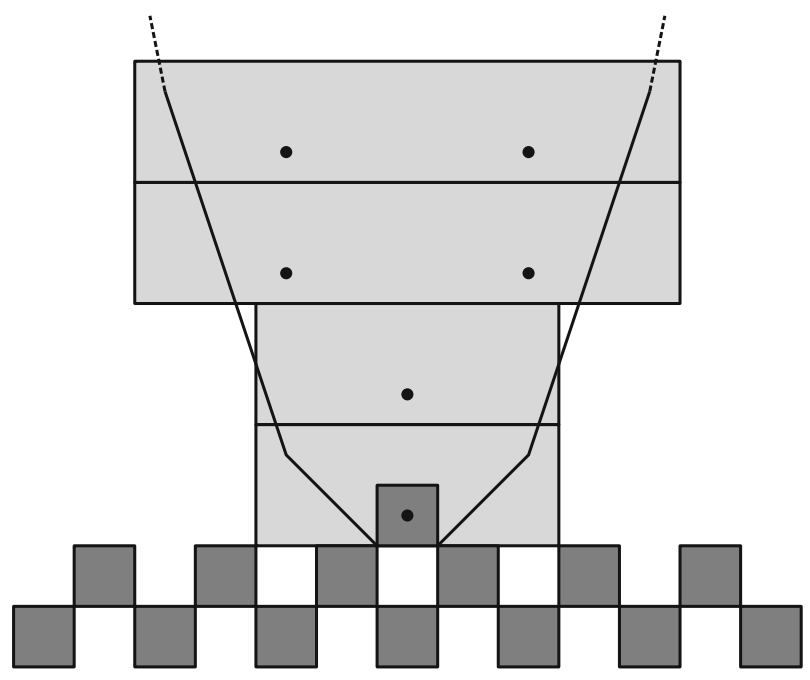

Fig. 33 Example of $C(\mathbf{i}), \mathbf{i} \in \mathcal{J}\left(\ell_{0}\right)$. The black dots represent the lattice points $\left( \pm b_{h}, 2 h\right)$

see Fig. 33.

Step 6: compatibility between different coverings. Here, we note that the family of sets $\left\{C(\mathbf{i}): \mathbf{i} \in \mathcal{J}\left(\ell_{m}\right), 0 \leq m \leq m_{1}, \mathbf{i} \in \mathcal{J}\left(\ell_{m_{1}}^{\prime}\right)\right\}$ is a covering of $E\left(\left\{\mathbf{i} \in \mathbb{Z}^{2}\right.\right.$ : $\left.\left.\inf _{\mathbf{j} \in Z(E)}\|\mathbf{i}-\mathbf{j}\|_{1}, i_{2} \geq i_{1}\right\}\right)$, which is the region of plane "outside" the edges as in Step 1. We point out that if case (ii) of Step 4 does not hold, then $\mathcal{J}\left(\ell_{m_{1}}^{\prime}\right)=A\left(\ell_{m_{1}}^{\prime}\right)=\emptyset$. Indeed, for every pair $\ell, \ell^{\prime} \in \mathcal{E}(E)$ with $\ell^{\prime}$ preceding $\ell$, the sets

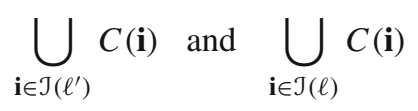

are non-overlapping and their union does not leave uncovered regions.

We denote by $a_{h}, b_{h}$ and $a_{h}^{\prime}, b_{h}^{\prime}$ the values defined in (5.30) corresponding to $\ell$ and $\ell^{\prime}$, respectively. We assume, for simplicity, that $\mathbf{j}^{L}=(0,0)$. Hence, every $\mathbf{i} \in \mathcal{J}(\ell)$ and $\mathbf{i}^{\prime} \in \mathcal{J}\left(\ell^{\prime}\right)$ are such that $i_{2}=2 h$ and $i_{2}^{\prime}=2 h+1+2 h_{0}$, where $h_{0}=0$ if $0 \leq s(\ell) \leq \frac{1}{3}$ and $2 h_{0}=r_{1}-r_{0}$ otherwise, where $r_{0}$ and $r_{1}$ are defined in Step 3. Therefore, in this coordinate system, the definition of $b_{h-h_{0}}^{\prime}$ reads

$$
b_{h-h_{0}}^{\prime}=\max \left\{h^{\prime} \in 2 \mathbb{Z}+1:\left(h^{\prime}+1,2 h+1\right) \in A\left(\ell^{\prime}\right)\right\} .
$$

Now, it is sufficient to note that if $Q\left(\left(a_{h}, 2 h\right)\right)=R^{\mathrm{ver}}\left(\left(a_{h}, 2 h\right)\right)$, then $a_{h+1}=a_{h}+2$, while if $Q\left(\left(a_{h}, 2 h\right)\right)=Q\left(\left(a_{h}, 2 h\right)\right)$, then $a_{h+1}=a_{h}$, as it immediately follows from (5.32) (see Fig. 34).

The covering of the regions projecting onto discrete edges $\ell \in \mathcal{E}(E)$ not fulfilling (5.29) can be obtained symmetrically; we use the notation $\mathcal{J}(\ell)$ and $C$ (i) to denote the sets obtained symmetrically as in (5.31) and (5.32), respectively. With $\mathcal{C}_{0}$, we denote the union of the set $\mathcal{S}_{0}$ defined in (5.34) and its symmetric analogs. 


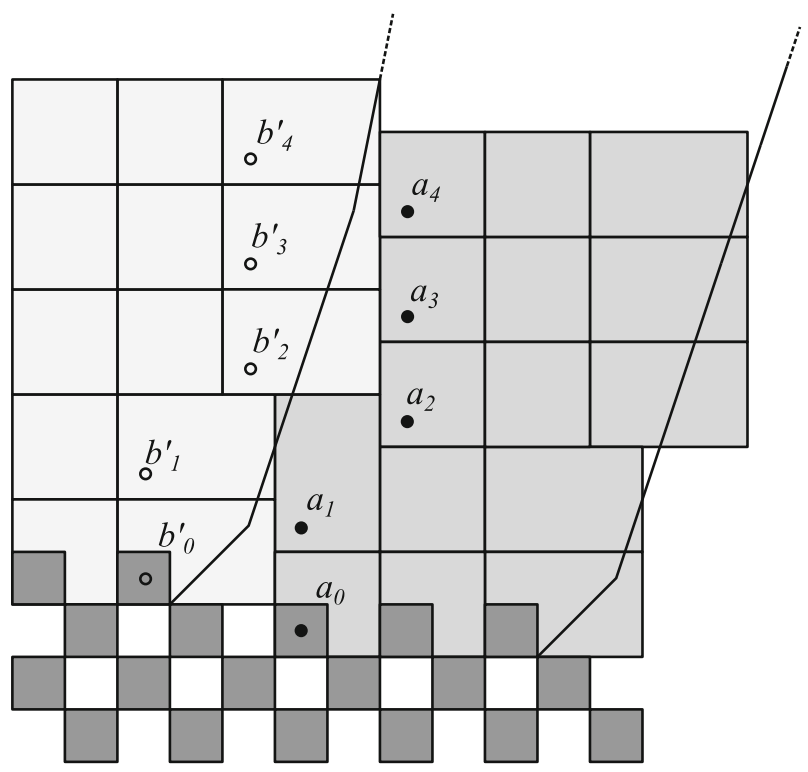

Fig. 34 Matching of the coverings outside a pair of adjacent discrete edges
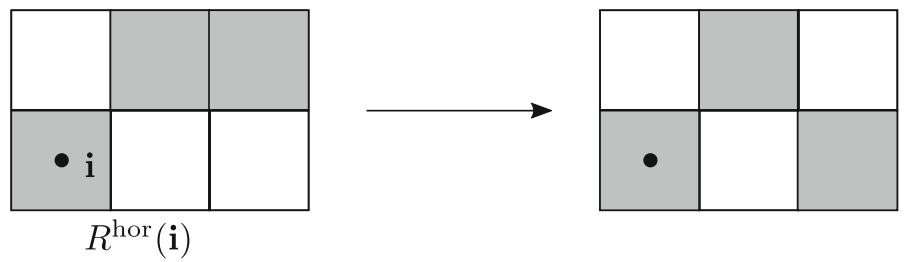

Fig. 35 The checkerboard configurations are energetically favorable inside each $R^{\text {hor }}(\mathbf{i})$

Step 7: local minimum problems on $C$ (i). As a next step, we prove that the configuration with minimal energy inside each tile $C(\mathbf{i})$ is the even checkerboard, for any $\mathbf{i} \in \mathcal{J}(\ell), \ell \in \mathcal{E}(E)$; i.e.,

$$
\mathcal{F}_{\alpha}^{\varphi}\left(E\left(\mathbb{Z}_{e}^{2}\right) \cap C(\mathbf{i}), E\right) \leq \mathcal{F}_{\alpha}^{\varphi}(F \cap C(\mathbf{i}), E)
$$

for every $F \in \mathcal{D}$, and the same for $\mathcal{C}_{0}$; i.e.,

$$
\mathcal{F}_{\alpha}^{\varphi}\left(E\left(\mathbb{Z}_{e}^{2}\right) \cap \mathcal{C}_{0}, E\right) \leq \mathcal{F}_{\alpha}^{\varphi}\left(F \cap \mathcal{C}_{0}, E\right)
$$

Indeed, if $C(\mathbf{i})=Q(\mathbf{i})$ from Remark 31, either (5.18) or (5.20) holds. Hence, by arguing as in the proof of Proposition 27, from (5.8) we get (5.36).

If $C_{h}(\mathbf{i})=R^{\text {hor }}(\mathbf{i})$, we can restrict the minimization in (5.36) to the checkerboard configurations. Indeed, if $\mathbf{j} \in Z\left(R^{\text {hor }}(\mathbf{i})\right)$ has a nearest-neighbor $\mathbf{j}^{\prime}$, then by suitably shifting one of them toward an "empty" location the corresponding variation of the energy is at most $-2+\alpha<0$ (see Fig. 35); the case $\alpha>2$ is trivial. Moreover, 


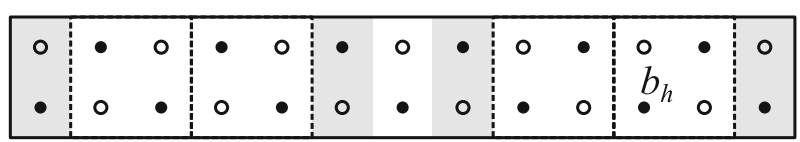

Fig. 36 The lattice points involved in (5.38). The black dots are points of $\mathbb{Z}_{e}^{2}$, and circles are points of $\mathbb{Z}_{o}^{2}$. The energy contribution of the even checkerboard in the white regions is negative

by the definition of $b_{h}$ we have that either (5.18) or (5.20) is satisfied, thence from Remark 32, (5.21) holds yielding (5.36). The cases of $C(\mathbf{i})=R^{\text {ver }}(\mathbf{i}), C(\mathbf{i})=R^{+}(\mathbf{i})$ and $C(\mathbf{i})=R^{-}$(i) can be treated analogously.

Now, consider the case $C(\mathbf{i})$ as in (5.35) with $\mathbf{i} \in \mathcal{J}\left(\ell_{0}\right)$. Reasoning as above, we can reduce minimum problem (5.36) to a comparison between the energies of the two checkerboards. Then, for every $h \in \mathbb{N}, k \in 2 \mathbb{Z}$ with $0<|k| \leq b_{h}$, the even checkerboard has minimum energy in $Q((k, 2 h))$, as above. Hence, $(5.36)$ is proved if

$$
\begin{aligned}
& \varphi(0,2 h)+2 \varphi(1,2 h+1)+2 d^{\varphi}\left(\left(b_{h}+2,2 h\right), E\right) \\
& \leq \varphi(0,2 h+1)+2 \varphi(1,2 h)+2 d^{\varphi}\left(\left(b_{h}+2,2 h+1\right), E\right)
\end{aligned}
$$

that is,

$$
\varphi(1,2 h+1)+d^{\varphi}\left(\left(b_{h}+2,2 h\right), E\right) \leq \frac{1}{2}+\varphi(1,2 h)+d^{\varphi}\left(\left(b_{h}+2,2 h+1\right), E\right),
$$

see Fig. 36. If $\left(b_{h}+2,2 h+1\right) \notin A\left(\ell_{0}\right)$, the inequality above is trivial, since $d^{\varphi}\left(\left(b_{h}+\right.\right.$ $2,2 h), E) \leq \varphi(1,2 h)$ and $d^{\varphi}\left(\left(b_{h}+2,2 h\right), E\right) \leq \varphi(1,2 h+1)$. If, instead, $\left(b_{h}+\right.$ $2,2 h+1) \in A\left(\ell_{0}\right),(5.38)$ reduces to

$$
\varphi(1,2 h+1)+\varphi\left(b_{h}+2,2 h\right) \leq \frac{1}{2}+\varphi(1,2 h)+\varphi\left(b_{h}+2,2 h+1\right),
$$

which holds from (5.8) and (H3).

Reasoning as in point (c) of the proof of Proposition 27, there holds

$$
\mathcal{F}_{\alpha}^{\varphi}\left(E\left(\mathbb{Z}_{e}^{2}\right) \cap \bigodot_{0}, E\right) \leq \mathcal{F}_{\alpha}^{\varphi}\left(F \cap \bigodot_{0}, E\right) .
$$

As a final remark, we note that for every $\mathbf{i} \in E\left(\mathbb{Z}_{e}^{2}\right)$ such that $d^{\varphi}(\mathbf{i}, E)>\frac{4}{\alpha}$ the variation of removing $q(\mathbf{i})$ is negative; hence,

$$
\underset{E^{\prime} \supset E}{\operatorname{argmin}} \mathcal{F}_{\alpha}^{\varphi}\left(E^{\prime}, E\right) \subset E\left(\left\{\mathbf{i} \in \mathbb{Z}^{2}: d^{\varphi}(\mathbf{i}, E)<\frac{4}{\alpha}\right\}\right) .
$$

Step 8: conclusion. Set

$$
\mathcal{J}:=\left(\bigcup_{\ell \in \mathcal{E}(E)} \mathcal{J}(\ell)\right) \cap\left\{\mathbf{i} \in \mathbb{Z}^{2}: d^{\varphi}(\mathbf{i}, E)<\frac{4}{\alpha}\right\}
$$


An analogous argument as that in the proof of Proposition 27 (see (5.12)) shows that

$$
\mathcal{F}_{\alpha}^{\varphi}\left(E^{\prime}, E\right) \geq \sum_{\mathbf{i} \in \mathcal{J}} \mathcal{F}_{\alpha}^{\varphi}\left(E^{\prime} \cap C(\mathbf{i}), E\right)+\mathcal{F}_{\alpha}^{\varphi}\left(E^{\prime} \cap \mathcal{C}_{0}, E\right)
$$

for every $E^{\prime} \supset E, E^{\prime} \in \mathcal{D}$. By virtue of Step 7, we get

$$
\min _{E^{\prime} \supset E} \mathcal{F}_{\alpha}^{\varphi}\left(E^{\prime}, E\right) \geq \sum_{\mathbf{i} \in \mathcal{J}} \mathcal{F}_{\alpha}^{\varphi}\left(E\left(\mathbb{Z}_{e}^{2}\right) \cap C(\mathbf{i}), E\right)+\mathcal{F}_{\alpha}^{\varphi}\left(E\left(\mathbb{Z}_{e}^{2}\right) \cap \mathcal{C}_{0}, E\right)=\mathcal{F}_{\alpha}^{\varphi}(E(\mathcal{J} \cup Z(E)), E)
$$

which implies that the ground state of the energy is achieved by the even checkerboard configuration. Lastly, the monotonicity constraint yields the uniqueness of the solution.

We will apply Proposition 30 iteratively to each $E=E_{\alpha}^{k}, k \geq 1$ in order to characterize the solutions of the recursive scheme (5.2) (see Theorem 38). Indeed, as shown with Proposition 27, the first step $E_{\alpha}^{1}$ coincides with $E\left(B_{\frac{4}{\alpha}}^{\varphi} \cap \mathbb{Z}_{e}^{2}\right)$ which satisfies the symmetry conditions (5.14) and, thanks to the following Lemma, the non-degeneracy condition (2.3).

Lemma 35 If $\varphi$ satisfies $(\mathrm{H} 1)$ and $(\mathrm{H} 2)$, then for every $r>2$ the set $E=B_{r}^{\varphi} \cap \mathbb{Z}_{e}^{2}$ satisfies (2.3).

Proof By the symmetric assumption (H1), we can restrict our analysis to points $\mathbf{i} \in$ $\partial^{\text {eff }} E$ with $i_{2} \geq i_{1} \geq 0$. We subdivide the proof into two cases. If $i_{1}=0$, then $i_{2}>0$ from $(\mathrm{H} 2)$ and the condition $r>2$. Since $(0,0) \in Z(E)$, we have that $\left(0, i_{2}-2\right) \in Z(E)$. By $(\mathrm{H} 1)$, the point $\left( \pm i_{2}, 0\right) \in Z(E)$; then, by the $\mathbb{Z}_{e}^{2}$-convexity of $E$ we get that $\left( \pm 1, i_{2}-1\right) \in Z(E)$. Since, for every $\mathbf{i}^{\prime}$ with $i_{2}^{\prime}>i_{2}, \mathbf{i}^{\prime} \notin Z(E)$, thus $\mathbf{i}$ is non-degenerate. If, instead, $i_{1}>0$, for every $\mathbf{j} \in Z(E)$ such that $\|\mathbf{j}-\mathbf{i}\|_{1} \leq 2$, by the symmetry with respect to the coordinate axes of $\varphi$ we get that $\left(-j_{1}, j_{2}\right)$, $\left(j_{1},-j_{2}\right) \in Z(E)$. The $\mathbb{Z}_{e}^{2}$-convexity and the fact that $(0,0) \in Z(E)$ yield that $\left(j_{1}-2, j_{2}\right),\left(j_{1}, j_{2}-2\right),\left(j_{1}+1, j_{2}-2\right) \in Z(E)$. This implies that $\mathbf{i}$ is non-degenerate.

We conclude this section with some examples clarifying the role of compatibility assumption (5.13) and non-degeneracy condition (H2).

Example 36 Consider $\varphi$ the Euclidean norm, and set $\alpha=0.7$. Then, the resulting $E_{\alpha}^{1}$ complies with (5.13) and the lattice sets $A(\ell)$ fulfill (5.25), as it can be noted in Fig. 37.

If we choose instead $\varphi=\|\cdot\|_{3}$ and $\alpha=0.71$, the compatibility condition (5.13) is violated for $E_{\alpha}^{1}$ as shown in Fig. 38. This also provides an example in which (5.25) is not satisfied; hence, the indices $a_{h}$ and $b_{h}$ introduced in Step 2 of Proposition 30 are not well defined.

Example 37 (one-dimensional motion) We consider an absolute norm which does not satisfy the normalization assumption $\varphi(1,0)=\varphi(0,1)=1$; that is,

$$
\varphi(\mathbf{x})=\left|x_{1}\right|+2\left|x_{2}\right|, \quad \text { for every } \mathbf{x} \in \mathbb{R}^{2},
$$



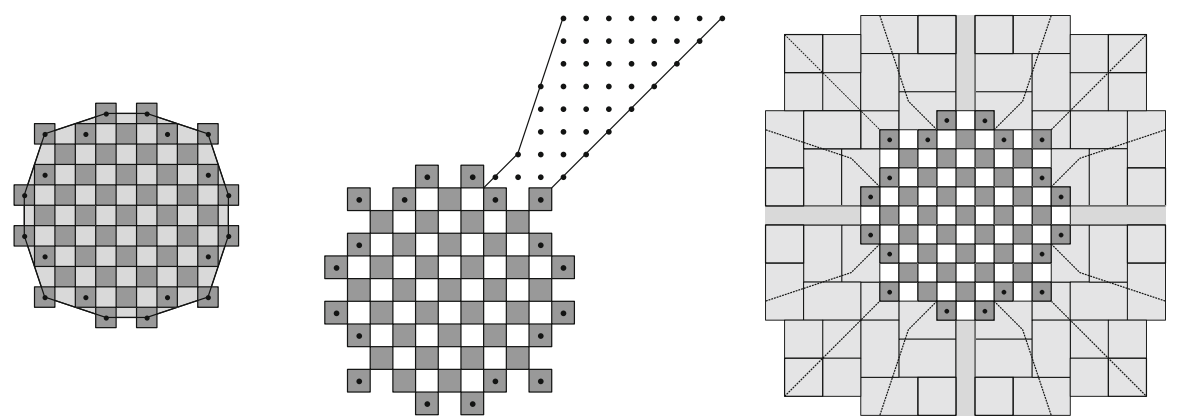

Fig. 37 From the left, the set $E_{\alpha}^{1}$ and the polygon $\operatorname{conv}\left(Z\left(E_{\alpha}^{1}\right)\right)$, the lattice sets $A(\ell), \ell \in \mathcal{E}(E)$ and, lastly, the corresponding covering

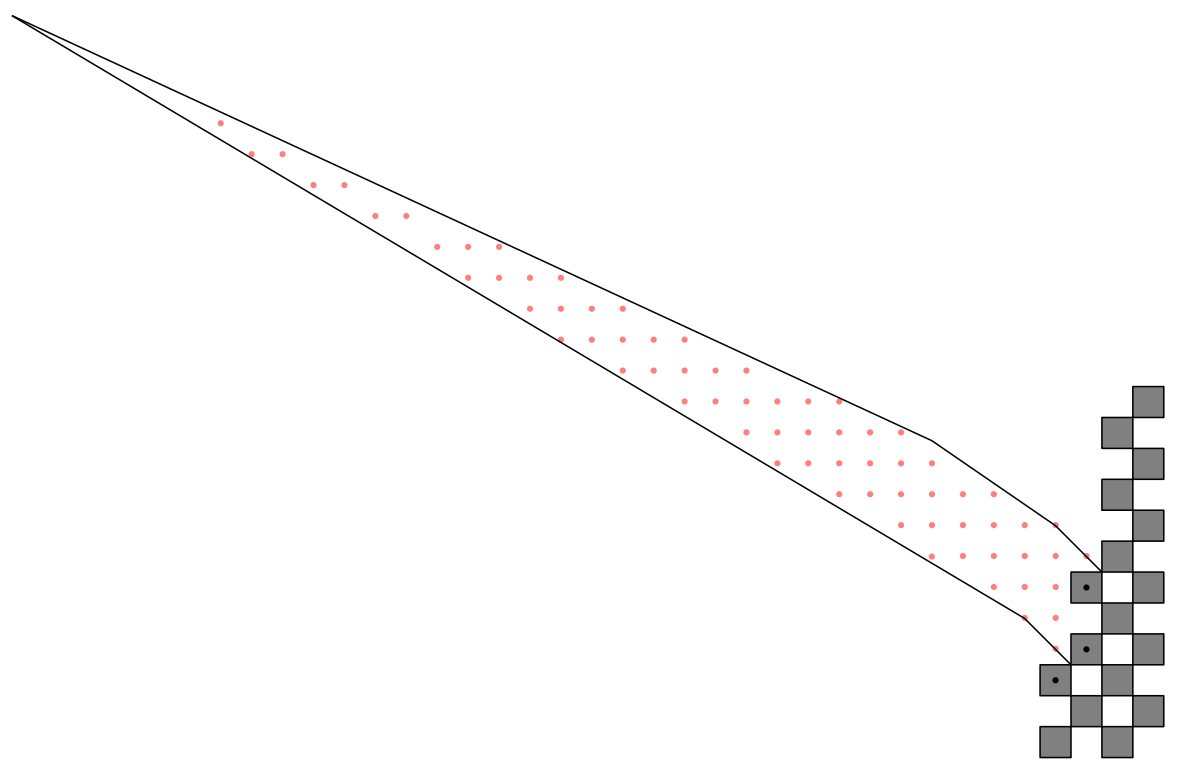

Fig. 38 The discrete edge $\ell$ represented with black dots does not satisfy the compatibility condition (5.13). The red dots denote the points of $A(\ell)$ which does not comply with (5.25). (Color figure online)
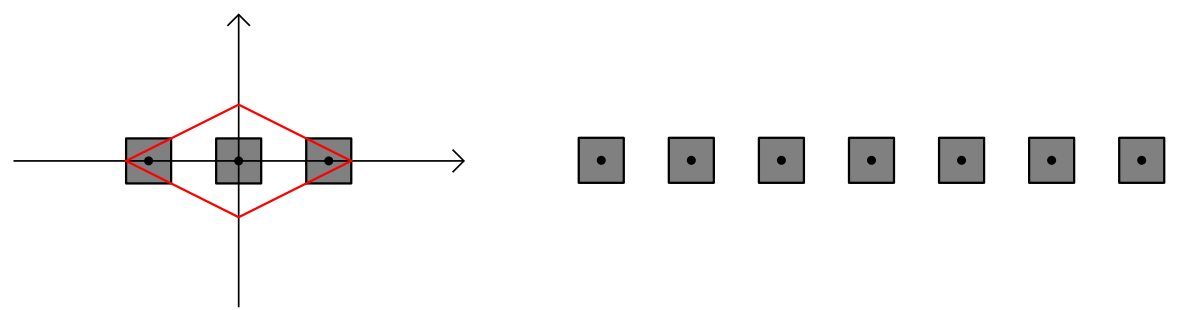

Fig. 39 On the left the set $E_{\alpha}^{1}$ and the boundary of $B_{\frac{4}{\alpha}}^{\varphi}$ in red. On the right the discrete solution $E_{\alpha}^{k}$ after two steps. (Color figure online) 
and take $\frac{4}{3}<\alpha<2$. Then, Proposition 27 applies in this case and gives

$$
E_{\alpha}^{1}=q((-2,0)) \cup q((0,0)) \cup q((2,0))
$$

Even though Proposition 30 cannot be applied on such set, it is straightforward to see in a direct way that the solution of $5.2\left\{E_{\alpha}^{k}\right\}$ is given by

$$
E_{\alpha}^{k}=\bigcup_{h=0}^{k}(q((-2 h, 0)) \cup q((2 h, 0))), k \geq 0,
$$

see Fig. 39. The resulting minimizing movement will be the family of horizontal line segments

$$
E(t)=\lim _{\varepsilon \rightarrow 0} E_{\varepsilon, \tau}(t)=\lim _{\varepsilon \rightarrow 0} \varepsilon E_{\alpha}^{\left\lfloor\frac{t}{\tau}\right\rfloor}=[-2 \alpha t, 2 \alpha t] \times\{0\}, t \geq 0 .
$$

\subsection{Nucleation and Growth of a Set}

By virtue of Proposition 30, we can characterize the time-discrete flow $\left\{E_{\alpha}^{k}\right\}_{k \geq 0}$ solution of (5.2). This evolution admits an alternative interpretation, based on a geometric iterative process that we will call nucleation of the initial set. Indeed, the set of centers of the $k$ th step of the discrete evolution $Z\left(E_{\alpha}^{k}\right)$ can be obtained from that of the previous step $Z\left(E_{\alpha}^{k-1}\right)$ by adding (in the Minkowski sense) the nucleus $\mathcal{N}_{\alpha}^{\varphi}$ (see Definition $28)$; i.e., a lattice set that characterizes the motion.

Theorem 38 Let $\varphi$ be a symmetric absolute normalized norm satisfying (H3), and let $\alpha>0$ be such that $\alpha \notin \Lambda^{\varphi}$. If $E\left(\mathcal{N}_{\alpha}^{\varphi}\right)$ satisfies assumption (5.13), then there exists a unique discrete solution $\left\{E_{\alpha}^{k}\right\}$ of (5.2) which is given, for any $k \geq 1$, by

$$
Z\left(E_{\alpha}^{k}\right)=\underbrace{\mathcal{N}_{\alpha}^{\varphi}+\cdots+\mathcal{N}_{\alpha}^{\varphi}}_{k \text {-times }} .
$$

In particular, $E_{\alpha}^{k} \in \mathcal{A}_{\text {conv }}^{e}$ for every $k \geq 1$.

Proof We first note that for a lattice set $\mathcal{J}$ such that $E(\mathcal{J})$ belongs to $\mathcal{A}_{\text {conv }}^{e}$ and satisfies (5.13)

$$
E(\underbrace{\mathcal{J}+\mathcal{J}+\cdots+\mathcal{J}}_{m \text {-times }}) \in \mathcal{A}_{\text {conv }}^{e} \text { still satisfies }(5.13), \text { for every } m \in \mathbb{N} .
$$

It will suffice to show (5.40) for $m=2$, as the claim for $m \geq 3$ will follow by an induction argument on the number $m$ of the summands. Setting $Q:=\operatorname{conv}(\mathcal{J})$, property (2.8) with $\Lambda=\mathbb{Z}_{e}^{2}$ and $m=2$ reads as $\left(\mathcal{Q} \cap \mathbb{Z}_{e}^{2}\right)+\left(\mathcal{Q} \cap \mathbb{Z}_{e}^{2}\right)=2 \mathcal{Q} \cap \mathbb{Z}_{e}^{2}$, yielding that $E(\mathcal{J}+\mathcal{J}) \in \mathcal{A}_{\text {conv }}^{e}$. Moreover, a property equivalent to (5.13) is that all the discrete vertices of $E(\mathcal{J})$ belongs to $\partial \mathcal{Q}$. This implies that the set of outward unit 
normal vectors of $Q$ coincide with the set of (discrete) outward unit normal vectors of $E(\mathcal{J})$. In particular, every edge $l$ of $Q$ identifies a finite chain of discrete edges of $E(\mathcal{J})$ having the same unit normal vector $v(l)$. This fact depends only on $v(l)$ and not on the length of $l$. Proposition 10 with $A=B=\mathcal{Q}$ implies that the set of outward unit normal vectors of $2 Q$ coincide with that of $Q$. Hence, the edge $l+l$ of $2 Q$ corresponds to a chain of a finite number of discrete edges of $E(\mathcal{J}+\mathcal{J})$ having the same unit normal vector $v(l)$. The $\mathbb{Z}_{e}^{2}$-convexity of $E(\mathcal{J}+\mathcal{J})$ implies $(5.13)$.

Going back to the proof of (5.39), we argue by induction on the step $k$. By Proposition 27 and Lemma 35, $Z\left(E_{\alpha}^{1}\right)=\mathcal{N}_{\alpha}^{\varphi}$ complies with all the assumptions on $E$ of Proposition 30. Now, let $k \geq 2$ and assume that

$$
Z\left(E_{\alpha}^{k-1}\right)=\underbrace{\mathcal{N}_{\alpha}^{\varphi}+\cdots+\mathcal{N}_{\alpha}^{\varphi}}_{(k-1) \text {-times }} .
$$

For what remarked in (5.40), all the hypotheses of Proposition 30 are satisfied. Then, taking into account (5.16) with $E=E_{\alpha}^{k-1}$, we have that

$$
Z\left(E_{\alpha}^{k}\right)=Z\left(E_{\alpha}^{k-1}\right)+\mathcal{N}_{\alpha}^{\varphi}
$$

Indeed, setting $\mathcal{J}_{k}:=Z\left(E_{\alpha}^{k-1}\right)+\mathcal{N}_{\alpha}^{\varphi}$, we have

$$
\max \left\{d^{\varphi}(\mathbf{i}, \mathbf{j}): \mathbf{i} \in \mathcal{J}_{k}, \mathbf{j} \in Z\left(E_{\alpha}^{k-1}\right)\right\} \leq \max _{\mathbf{i} \in \mathcal{N}_{\alpha}^{\varphi}} \varphi(\mathbf{i})<\frac{4}{\alpha},
$$

and this shows that $\mathcal{J}_{k} \subseteq Z\left(E_{\alpha}^{k}\right)$. On the other hand, if $\mathbf{i} \in Z\left(E_{\alpha}^{k}\right)$, there exist $\mathbf{i}^{\prime} \in$ $Z\left(E_{\alpha}^{k-1}\right)$ and $\mathbf{i}^{\prime \prime} \in \mathcal{N}_{\alpha}^{\varphi}$ such that $\mathbf{i}=\mathbf{i}^{\prime}+\mathbf{i}^{\prime \prime}$. This comes by noting that by (5.16) there exists $\mathbf{i}^{\prime} \in Z\left(E_{\alpha}^{k-1}\right)$ such that $\varphi\left(\mathbf{i}-\mathbf{i}^{\prime}\right)=d^{\varphi}\left(\mathbf{i}, E_{\alpha}^{k-1}\right)<\frac{4}{\alpha}$, thus $\mathbf{i}^{\prime \prime}=\mathbf{i}-\mathbf{i}^{\prime} \in \mathcal{N}_{\alpha}^{\varphi}$. This yields (5.39). Moreover, again by (5.40) we get that the Minkowski sum in (5.41) preserves assumption (5.13), so $E_{\alpha}^{k}$ still satisfies (5.13) and the thesis is proved.

\section{The Limit Motion}

In this section, we characterize the minimizing movements of scheme (3.4) as $\tau, \varepsilon \rightarrow 0$ in the critical regime $\varepsilon=\alpha \tau$ for any positive value of the parameter $\alpha$ outside the singular set $\Lambda^{\varphi}$, under the assumption that $E\left(\mathcal{N}_{\alpha}^{\varphi}\right)$ complies with (5.13).

As already explained at the beginning of Sect. 5, we also prove the existence of a value for $\alpha$ depending only on the chosen norm $\varphi$, above which every minimizing movement is trivial. For every $\alpha$ below the pinning threshold, instead, the limit motion is a family of expanding sets, nucleating from the origin with constant velocity, as the limit set $E(t)$ turns out to be a dilation of the (renormalized) polygon

$$
P_{\alpha}^{\varphi}:= \begin{cases}\left(\max _{\mathbf{i} \in \mathcal{N}_{\alpha}^{\varphi}} i_{1}\right)^{-1} \operatorname{conv}\left(\mathcal{N}_{\alpha}^{\varphi}\right) & \text { if } \mathcal{N}_{\alpha}^{\varphi} \neq\{(0,0)\} \\ \{(0,0)\} & \text { if } \mathcal{N}_{\alpha}^{\varphi}=\{(0,0)\}\end{cases}
$$


Note that $\max _{\mathbf{i} \in \mathcal{N}_{\alpha}^{\varphi}} i_{1} \in\left\{2\left\lfloor\frac{2}{\alpha}\right\rfloor,\left\lfloor\frac{4}{\alpha}\right\rfloor\right\}$, from the definition of $\mathcal{N}_{\alpha}^{\varphi}$ and the fact that $\varphi\left(i_{1}, 0\right)=i_{1}, i_{1} \in \mathbb{N}$.

Theorem 39 Let $\varphi$ be a symmetric absolute normalized norm satisfying (H3), let $\alpha>0$ be given such that $\alpha \notin \Lambda^{\varphi}$, and let $\mathcal{F}_{\varepsilon, \tau}^{\varphi}$ be defined by (3.3). Let $\mathcal{N}_{\alpha}^{\varphi}$ be as in Definition 28. If the set $E\left(\mathcal{N}_{\alpha}^{\varphi}\right)$ satisfies assumption (5.13), then there exists a unique minimizing movement $E:[0,+\infty) \rightarrow X$ for the scheme (3.4) at regime $\varepsilon=\alpha \tau$ defined by

$$
E(t)=v_{\alpha}^{\varphi} t P_{\alpha}^{\varphi} \text { for every } t \geq 0
$$

where $P_{\alpha}^{\varphi}$ is defined in (6.1) and $v_{\alpha}^{\varphi}=\alpha \max _{\mathbf{i} \in \mathcal{N}_{\alpha}^{\varphi}} i_{1}$. Moreover, there exists a unique discrete solution $E_{\varepsilon, \tau}(t)$ of scheme (3.4) at regime $\varepsilon=\alpha \tau$ and there holds

$$
\chi_{E_{\varepsilon, \tau}(t)} \stackrel{*}{\rightarrow} \frac{1}{2} \chi_{E(t)}, \quad \text { for every } t \geq 0 \text { as } \varepsilon \rightarrow 0
$$

Proof By a scaling argument, for every discrete solution $E_{\varepsilon, \tau}^{k}$ of (3.4) in the regime $\varepsilon=\alpha \tau$ we have $E_{\varepsilon, \tau}^{k}=\varepsilon E_{\alpha}^{k}$ for every $k \geq 0$, where $E_{\alpha}^{k}$ denotes a discrete solution of (5.2). Then, by Theorem 38 there exists a unique minimizing movement of scheme (3.4) at regime $\varepsilon=\alpha \tau$. Since, by Proposition 12 and (6.1),

$$
\underbrace{\mathcal{N}_{\alpha}^{\varphi}+\cdots+\mathcal{N}_{\alpha}^{\varphi}}_{k \text {-times }}=\left(k \frac{v_{\alpha}^{\varphi}}{\alpha} P_{\alpha}^{\varphi}\right) \cap \mathbb{Z}_{e}^{2},
$$

we get that $\operatorname{conv}\left(Z_{\varepsilon}\left(E_{\varepsilon, \tau}(t)\right)\right)=\varepsilon \frac{v_{\alpha}^{\varphi}}{\alpha}\left\lfloor\frac{\alpha t}{\varepsilon}\right\rfloor P_{\alpha}^{\varphi}$. Moreover, noting that $d_{\mathcal{H}}\left(F, \operatorname{conv}\left(Z_{\varepsilon}(F)\right)\right)<\varepsilon$ for any $F \in \mathcal{A}_{\varepsilon}$, we get

$$
d_{\mathcal{H}}\left(E_{\varepsilon, \tau}(t), v_{\alpha}^{\varphi} t P_{\alpha}^{\varphi}\right)<\varepsilon+v_{\alpha}^{\varphi}\left(t-\frac{\varepsilon}{\alpha}\left\lfloor\frac{\alpha t}{\varepsilon}\right\rfloor\right)
$$

which tends to zero as $\varepsilon \rightarrow 0$, for any $t \geq 0$, whence (6.2) follows. Eventually, from the fact that $\left|E_{\varepsilon, \tau}(t) \cap A\right| \rightarrow \frac{1}{2}|A|$ as $\varepsilon \rightarrow 0$ for any open set $A \subset E(t)$, we get (6.3).

Definition 40 (pinning threshold) We define the pinning threshold of the motion obtained by solving scheme (3.4) as

$\alpha_{\varphi}:=\inf \left\{\alpha>0: E^{\alpha}(t) \equiv\{(0,0)\}\right.$ for every $E^{\alpha}$ minimizing movement of $\left.(3.4)\right\}$

It turns out that $\alpha_{\varphi}$ is related to the singular set $\Lambda^{\varphi}$ defined in (5.4) as follows.

Proposition 41 The pinning threshold is given by $\alpha_{\varphi}=\frac{4}{\varphi(1,1)}=\max \Lambda^{\varphi}$.

Proof We note that $B_{\frac{4}{\alpha}}^{\varphi} \cap \mathbb{Z}_{e}^{2}=\{(0,0)\}$ if and only if $\alpha>\frac{4}{\varphi(1,1)}$, and thus, Proposition 27 yields the result. 
Remark 42 The results of Theorems 38 and 39 can be extended to solutions of a minimizing-movement scheme with a more general initial datum $E^{0}$. Indeed, let $E_{\text {disc }}^{0} \in \mathcal{A}_{\text {conv }}^{e}$ be a set satisfying (2.3), (5.13) and (5.14). We can apply Proposition 30 with $E^{\prime}=E_{\text {disc }}^{0}$ obtaining the first step of the discrete solution corresponding to scheme (5.2) with $E_{\alpha}^{0}=E_{\text {disc }}^{0}$. Then, if $E\left(\mathcal{N}_{\alpha}^{\varphi}\right)$ satisfies assumption (5.13), from the same arguments of the proof of Theorem 38 there exists a unique discrete solution of the scheme

$$
\left\{\begin{array}{l}
E_{\alpha}^{0}=E_{\text {disc }}^{0} \\
E_{\alpha}^{k+1} \in \underset{E^{\prime} \in \mathcal{D}, E^{\prime} \supset E}{\operatorname{argmax}} \mathcal{F}_{\alpha}^{\varphi}\left(E^{\prime}, E_{\alpha}^{k}\right) \quad k \geq 1
\end{array}\right.
$$

which is given, for any $k \geq 1$, by $Z\left(E_{\alpha}^{k}\right)=Z\left(E_{\text {disc }}^{0}\right)+\underbrace{\mathcal{N}_{\alpha}^{\varphi}+\cdots+\mathcal{N}_{\alpha}^{\varphi}}_{k \text {-times }}$.

We therefore obtain a limit result analogous to that of Theorem 39, provided the initial datum $E^{0}$ can be approximated by a sequence of admissible sets $E_{\varepsilon_{j}}^{0} \in \mathcal{A}_{\varepsilon_{j}}$ whose rescaled sets $\frac{1}{\varepsilon_{j}} E_{\varepsilon_{j}}^{0} \in \mathcal{A}_{\text {conv }}^{e}$ satisfy (2.3), (5.13) and (5.14). This implies, in particular, that $E^{0}$ must be a convex symmetric set with respect to coordinated axes and bisectors $x_{1}= \pm x_{2}$. Then, there exists (up to subsequences) a minimizing movement $E:[0,+\infty) \rightarrow X$ for the scheme

$$
\left\{\begin{array}{l}
E_{\varepsilon, \tau}^{0}=E_{\varepsilon}^{0} \\
E_{\varepsilon, \tau}^{k+1} \in \underset{E^{\prime} \in \mathcal{D}_{\varepsilon}, E^{\prime} \supset E_{\varepsilon, \tau}^{k}}{\operatorname{argmin}}
\end{array} \mathcal{F}_{\varepsilon, \tau}^{\varphi}\left(E^{\prime}, E\right) \quad k \geq 1\right.
$$

at regime $\varepsilon=\alpha \tau$ defined by

$$
E(t)=E^{0}+v_{\alpha}^{\varphi} t P_{\alpha}^{\varphi} \text { for every } t \geq 0
$$

Moreover, there exists a unique discrete flat flow $E_{\varepsilon_{j}, \tau_{j}}(t)$ of the scheme (6.5) along the sequence $\varepsilon_{j}=\alpha \tau_{j}$ and there holds $\chi_{E_{\varepsilon_{j}, \tau_{j}}(t)} \stackrel{*}{\rightarrow} \frac{1}{2} \chi_{E(t)}$ for every $t \geq 0$ as $j \rightarrow+\infty$.

\subsection{Examples of Explicit Evolutions}

We continue our analysis by providing several examples of minimizing movements that can be completely characterized, which exhibit interesting phenomena that may appear due to the discrete nature of our problem.

Example 43 (the $\ell^{\infty}$-norm) The solutions of the unconstrained scheme (3.5) have already been analyzed in any dimension in the case $\varphi=\|\cdot\|_{\infty}$ in Braides and Scilla (2013b), where it has been proved that every step of the discrete evolution is an even $\varepsilon$-checkerboard (see Fig. 40). Thus, solutions of (3.4) and (3.5) coincide. The singular set (5.4) corresponds to $\Lambda^{\varphi}=\left\{\frac{4}{n}\right\}_{n \in \mathbb{N}}$ and the pinning threshold is $\alpha_{\varphi}=4$. Here, since $\mathcal{N}_{\alpha}^{\varphi}=\left[-\frac{4}{\alpha}, \frac{4}{\alpha}\right]^{2} \cap \mathbb{Z}_{e}^{2}$ for every $\alpha \notin \Lambda^{\varphi}, E\left(\mathcal{N}_{\alpha}^{\varphi}\right)$ always fulfills (5.13). Therefore, 

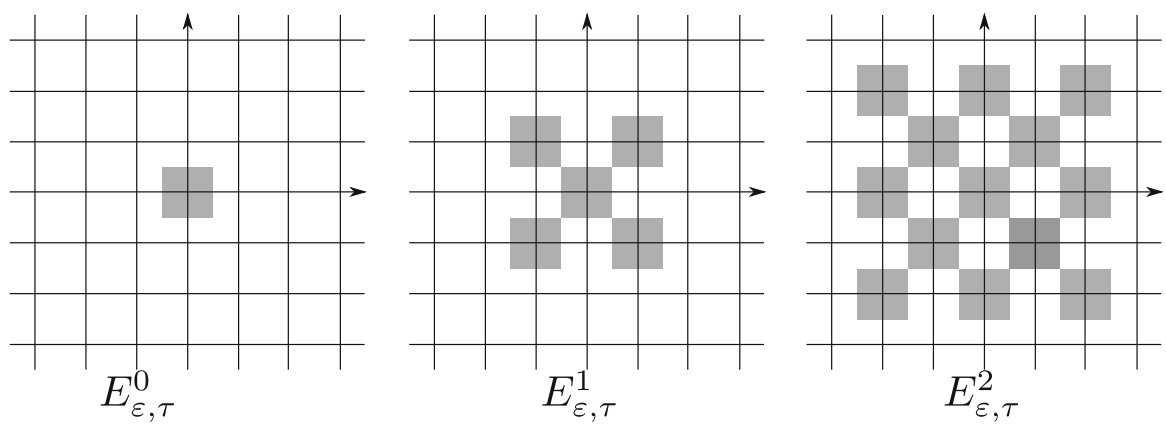

Fig. 40 Some steps of the discrete evolution
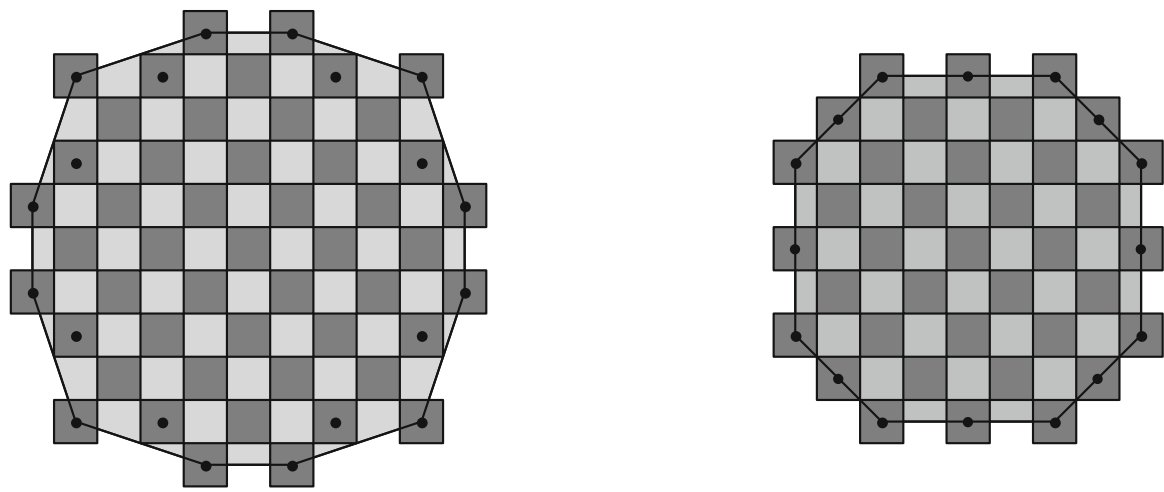

Fig. 41 For different choices of $\alpha$, the polygon $P_{\alpha}^{\varphi}$ may have different shapes

from Theorem 39, for every $\alpha \notin \Lambda^{\varphi}$ the minimizing movement is

$$
E(t)=\left[-\alpha\left\lfloor\frac{4}{\alpha}\right\rfloor t, \alpha\left\lfloor\frac{4}{\alpha}\right\rfloor t\right]^{2}, \quad \text { for every } t \geq 0
$$

We note that, for this choice of the norm $\varphi$, the polygon $P_{\alpha}^{\varphi}=[-1,1]^{2}$ does not depend on $\alpha$.

Example 44 ( $\alpha$-depending shape of $P_{\alpha}^{\varphi}$ ) Contrarily to the previous example, in the case of the Euclidean norm the polygon $P_{\alpha}^{\varphi}$ may change with $\alpha$ (see, for instance, Fig. 41 corresponding to $\alpha=0.85$ on the left and $\alpha=0.7$ on the right). Therefore, the limit motions corresponding to the two different values of $\alpha$ are not homothetic. This phenomenon may happen for those norms $\varphi$ whose balls are not polygons or are polygons having a unit normal vectors different from $(0, \pm 1),( \pm 1,0)$ or $\left( \pm \frac{1}{\sqrt{2}}, \pm \frac{1}{\sqrt{2}}\right)$.

Example 45 (the $\ell^{1}$-norm) We consider now $\varphi=\|\cdot\|_{1}$. Also in this case, as for the $\infty$-norm, the structure of $\varphi$ facilitates the analysis of the unconstrained scheme (3.5). 
Fig. 42 The picture clarifies the $2 \times 2$-square covering $\mathcal{S}_{o}(E)$ for a set $E$, whose boundary is marked by a bold black line. The darker $2 \times 2$-squares are, respectively, in $\oint_{O}^{c}(E)$, the lighter ones in $\mathcal{S}_{o}^{b}(E)$. The areas in white are those left uncovered

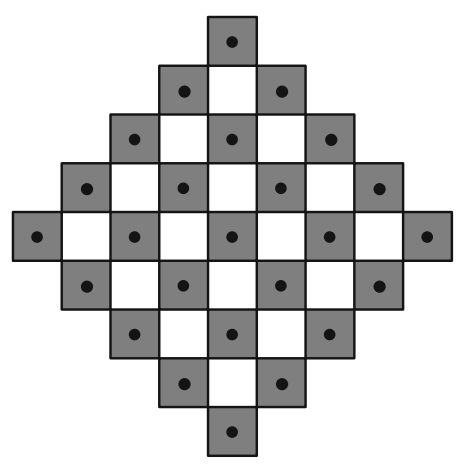

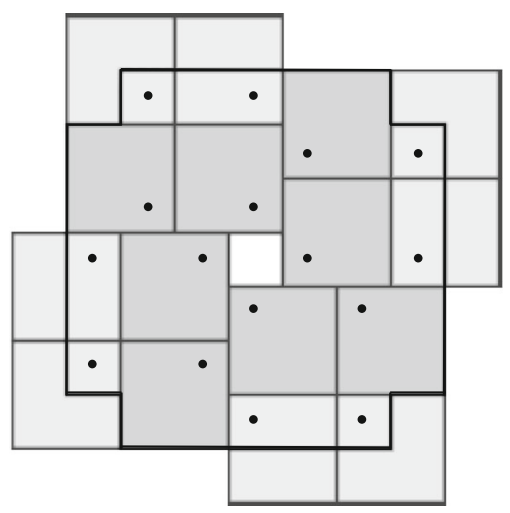

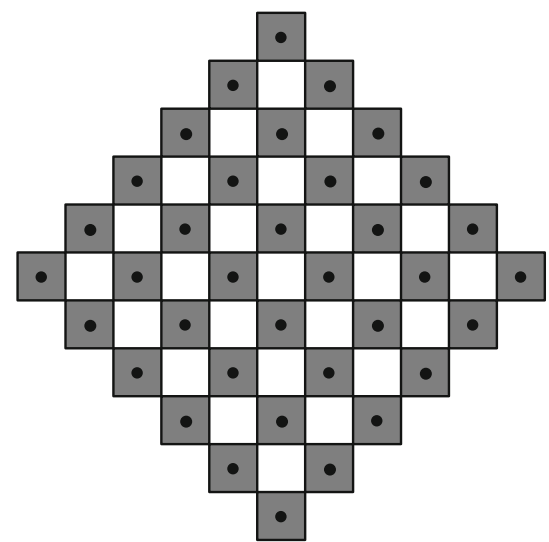

Fig. 43 An example of $E_{\alpha}^{e}$ (on the left) and $E_{\alpha}^{o}$ (on the right)

We then study the rescaled problem

$$
\left\{\begin{array}{l}
E^{0}=q \\
E_{\alpha}^{k+1} \in \underset{E^{\prime} \in \mathcal{D}}{\operatorname{argmin}} \mathcal{F}_{\alpha}^{\varphi}\left(E^{\prime}, E\right) \quad k \geq 1,
\end{array}\right.
$$

where we separately examine the cases in which the minimizer of the first step contains $q$ or not. For this, in addition to $\mathcal{S}_{e}(E)$ of Definition 26 we introduce the family

$$
\mathcal{S}_{o}(E):=\left\{Q(\mathbf{j}): Q(\mathbf{j}) \cap E \neq \emptyset \text { and } j_{1} \text { even, } j_{2} \text { odd }\right\}
$$

which is a covering of $E \backslash q$ (see Fig. 42) and, accordingly, we consider the partition $\mathcal{S}_{o}(E)=\mathcal{S}_{o}^{b}(E) \cup \mathcal{S}_{o}^{c}(E)$.

Now, in the case of scheme (6.7) with the monotonicity constraint, Proposition 27 and (5.4) ensure that if $\alpha \notin\left\{\frac{2}{n}: n \in \mathbb{N}\right\}$, then $\underset{E \supset q}{\operatorname{argmin}} \mathcal{F}_{\alpha}^{\varphi}(E, q)=E\left(\mathbb{Z}_{e}^{2} \cap B_{\frac{4}{\alpha}}^{\varphi}\right)=: E_{\alpha}^{e}$. In the unconstrained case, an analogous argument as in the proof of Proposition 27, 

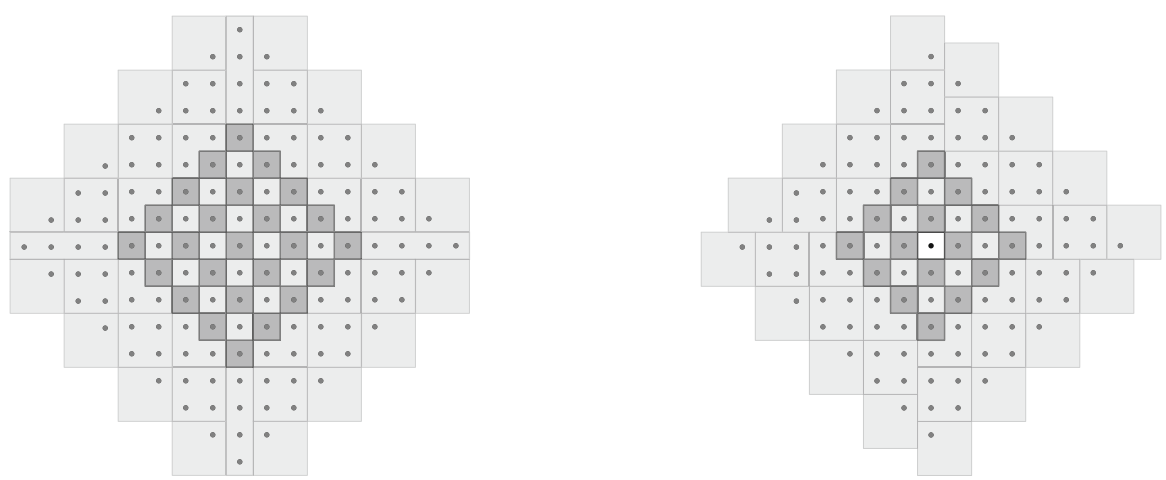

Fig. 44 On the left the covering $\oint_{e}\left(E\left(\mathcal{J}^{e}\right)\right)$ and the set $E_{\alpha}^{e}$. On the right the covering $\mathcal{S}_{o}\left(E\left(\mathcal{J}^{o}\right)\right)$ and the set $E_{\alpha}^{o}$. The darker dots represent $\mathcal{J}^{e}$ and $\mathcal{J}^{o}$, respectively

with $\mathcal{S}_{O}$ in place of $\mathcal{S}_{e}$, shows that if $\alpha \notin\left\{\frac{4}{2 n-1}: n \in \mathbb{N}\right\}$, then $\underset{E \not \supset q}{\operatorname{argmin}} \mathcal{F}_{\alpha}^{\varphi}(E, q)=$ $E\left(\mathbb{Z}_{o}^{2} \cap B_{\frac{4}{\alpha}}^{\varphi}\right)=: E_{\alpha}^{o}$. This proves that $E_{\alpha}^{1}$ is either an even or an odd checkerboard. We remark that $B_{r}^{\varphi}$ is a regular rhombus (of radius $r$ ) and so are the convex hulls of $Z\left(E_{\alpha}^{e}\right)$ and $Z\left(E_{\alpha}^{o}\right)$. The checkerboard sets $E_{\alpha}^{e}$ and $E_{\alpha}^{o}$ are pictured in Fig. 43.

The relevant point of this example is that, for this choice of the norm, the shape of the minimizers is very simple and the $2 \times 2$-square covering argument of Sect. 5.1 directly applies to the $k^{t h}$-step $E_{\alpha}^{k}, k \geq 1$, without any further adjustment. Moreover, it provides a covering of $\mathbb{R}^{2}$ (in the even case) or $\mathbb{R}^{2} \backslash q$ (in the odd case) and not only of $\mathbb{R}^{2} \backslash E\left(\operatorname{conv}\left(Z\left(E_{\alpha}^{k-1}\right)\right)\right)$, see Fig. 44 . Thus, the corresponding localization argument allows to study the unconstrained problem. Indeed, if $E_{\alpha}^{1}=E_{\alpha}^{e}$, then for every $Q(\mathbf{j}) \in \mathcal{S}_{e}\left(E\left(\mathcal{J}^{e}\right)\right)$ we get

$$
\begin{gathered}
\mathcal{F}_{\alpha}^{\varphi}\left(Q(\mathbf{j}) \cap E\left(\mathbb{Z}_{e}^{2}\right), E_{\alpha}^{1}\right)=\min _{E \in \mathcal{D}} \mathcal{F}_{\alpha}^{\varphi}\left(Q(\mathbf{j}) \cap E, E_{\alpha}^{1}\right), \\
\mathcal{F}_{\alpha}^{\varphi}\left(\mathcal{C}_{0} \cap E\left(\mathbb{Z}_{e}^{2}\right), E_{\alpha}^{1}\right) \leq \min _{E \in \mathcal{D}} \mathcal{F}_{\alpha}^{\varphi}\left(\bigodot_{0} \cap E, E_{\alpha}^{1}\right),
\end{gathered}
$$

whereas if $E_{\alpha}^{1}=E_{\alpha}^{o}$, then for every $Q(\mathbf{j}) \in \mathcal{S}_{o}\left(E\left(\mathcal{J}^{o}\right)\right)$ we get

$$
\mathcal{F}_{\alpha}^{\varphi}\left(Q(\mathbf{j}) \cap E\left(\mathbb{Z}_{o}^{2}\right), E_{\alpha}^{1}\right) \leq \min _{E \in \mathcal{D}} \mathcal{F}_{\alpha}^{\varphi}\left(Q(\mathbf{j}) \cap E, E_{\alpha}^{1}\right),
$$

where

$$
\mathcal{J}^{e}=\left\{\mathbf{i} \in \mathbb{Z}_{e}^{2}: d^{\varphi}\left(\mathbf{i}, E_{\alpha}^{1}\right)<\frac{4}{\alpha}\right\}, \quad \mathcal{J}^{o}=\left\{\mathbf{i} \in \mathbb{Z}_{o}^{2}: d^{\varphi}\left(\mathbf{i}, E_{\alpha}^{1}\right)<\frac{4}{\alpha}\right\}
$$

which gives that $Z\left(E_{\alpha}^{2}\right) \in\left\{\mathcal{J}^{e}, \mathcal{J}^{o}\right\}$. This yields, after an inductive argument, that $E_{\alpha}^{k}$ is either an even or an odd checkerboard. The parity of $E_{\alpha}^{k}$ will be determined by a comparison between the two possible (checkerboard) configurations. Nevertheless, a change of parity is eventually not energetically favorable. Indeed, assume $E_{\alpha}^{k}$ to be, 

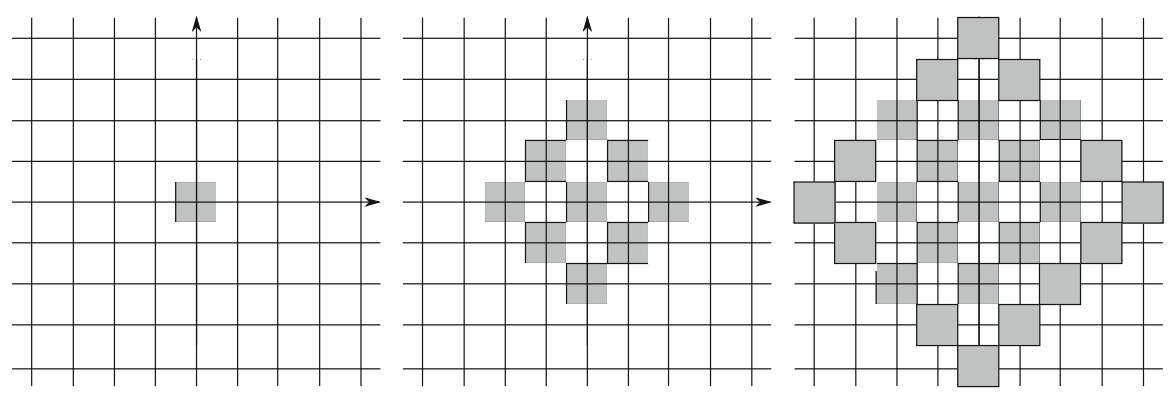

Fig. 45 Some steps of the even evolution

e.g., an even checkerboard and set $\mathcal{J}=\left\{\mathbf{i} \in \mathbb{Z}_{o}^{2}: d^{\varphi}\left(\mathbf{i}, E_{\alpha}^{k}\right)<\frac{4}{\alpha}\right\}$, we then get

$$
\begin{aligned}
\mathcal{F}_{\alpha}^{\varphi}\left(E(\mathcal{J}), E_{\alpha}^{k}\right)-\mathcal{F}_{\alpha}^{\varphi}\left(E_{\alpha}^{k}, E_{\alpha}^{k}\right) & \geq-4 \# Z(E(\mathcal{J}))+2 \alpha \# Z\left(E_{\alpha}^{k}\right)+4 \# Z\left(E_{\alpha}^{k}\right)+c \\
& \geq-8\left(\frac{4(k+1)}{\alpha}\right)^{2}+2 \alpha\left(\frac{4(k+1)}{\alpha}\right)^{2}+-8\left(\frac{4 k}{\alpha}\right)^{2}+c \\
& =-c^{\prime} k+c^{\prime \prime} k^{2}+c,
\end{aligned}
$$

for some positive constants $c, c^{\prime}, c^{\prime \prime}$. Since for $k$ large enough the contribution above is positive, for every fixed $\alpha \notin\left\{\frac{4}{n}\right\}_{n \in \mathbb{N}}$, there exists an index $k_{\alpha} \in \mathbb{N}$ such that

$$
Z\left(E_{\alpha}^{k}\right)=Z\left(E_{\alpha}^{k_{\alpha}}\right)+\underbrace{\mathcal{N}_{\alpha}^{\varphi}+\cdots+\mathcal{N}_{\alpha}^{\varphi}}_{\left(k-k_{\alpha}\right) \text {-times }}, \quad \text { for every } k \geq k_{\alpha}
$$

We can characterize the limit motion as follows. For every $\alpha>0$ such that $\alpha \notin$ $\left\{\frac{4}{n}\right\}_{n \in \mathbb{N}}$ there exists a unique minimizing movement of unconstrained scheme (3.5) $E:[0,+\infty) \rightarrow X$ and it satisfies

$$
E(t)=2 \alpha\left\lfloor\frac{2}{\alpha}\right\rfloor t \mathcal{R}, \quad \text { for every } t \geq 0,
$$

where $\mathcal{R}$ is the regular rhombus of radius 1 . Note that, by Theorem 39 , this coincides with the minimizing movement of the constrained scheme (3.4).

At least for the first step, the comparison between the energies of the two possible minimizers, i.e., $E_{\alpha}^{e}$ and $E_{\alpha}^{o}$, can be performed by a straightforward computation. This induces a partition into subintervals of the set $(0,+\infty) \backslash\left\{\frac{4}{n}: n \in \mathbb{N}\right\}$, wherein one configuration is energetically more favorable than the other one. Setting $R:=\left\lfloor\frac{4}{\alpha}\right\rfloor$, we get

$$
\mathcal{F}_{\alpha}^{\varphi}\left(E_{\alpha}^{e}, q\right)=-4\left(2\left\lfloor\frac{R}{2}\right\rfloor+1\right)^{2}+4 \alpha \sum_{j=1}^{\left\lfloor\frac{R}{2}\right\rfloor}(2 j)^{2},
$$




$$
\mathcal{F}_{\alpha}^{\varphi}\left(E_{\alpha}^{o}, q\right)=-4\left(2\left\lfloor\frac{R+1}{2}\right\rfloor\right)^{2}+4 \alpha \sum_{j=1}^{\left\lfloor\frac{R+1}{2}\right\rfloor}(2 j-1)^{2}+\alpha .
$$

After comparing the values in (6.10) and (6.11) we get that when $R$ is even

$$
\mathcal{F}_{\alpha}^{\varphi}\left(E_{\alpha}^{e}, q\right)<\mathcal{F}_{\alpha}^{\varphi}\left(E_{\alpha}^{o}, q\right) \text { if and only if } \alpha<\frac{4(2 R+1)}{2 R(R+1)-1},
$$

while when $R$ is odd

$$
\mathcal{F}_{\alpha}^{\varphi}\left(E_{\alpha}^{e}, q\right)<\mathcal{F}_{\alpha}^{\varphi}\left(E_{\alpha}^{o}, q\right) \text { if and only if } \alpha>\frac{4(2 R+1)}{2 R(R+1)+1} .
$$

Thus, for the following values of $\alpha$

$$
\alpha_{C}(R):= \begin{cases}\frac{4(2 R+1)}{2 R(R+1)+1} & \text { if } R \text { is odd, } \\ \frac{4(2 R+1)}{2 R(R+1)-1} & \text { if } R \text { is even, }\end{cases}
$$

the energies of the two checkerboards coincide and we also obtain that

$$
E_{\alpha}^{1}= \begin{cases}E_{\alpha}^{e} & \text { if } \alpha \in \bigcup_{h \geq 1}\left(\alpha_{C}(2 h+1), \alpha_{C}(2 h)\right) \cup\left(\alpha_{C}(1),+\infty\right), \\ E_{\alpha}^{o} & \text { if } \alpha \in \bigcup_{h \geq 0}\left(\alpha_{C}(2 h+2), \alpha_{C}(2 h+1)\right)\end{cases}
$$

In particular, (6.12) provides an example of a discrete solution having an oscillating behavior; that is, a change of parity from a step to another, at least from $E_{\alpha}^{0}=q$ to $E_{\alpha}^{1}=E_{\alpha}^{o}$.

In this case, the pinning threshold of unconstrained problem (3.5) is $\alpha_{p}=2$, as can be seen in formula (6.9). This is the same as that of the constrained problem (3.4), given by Proposition 41 . In the constrained problem, for every $\alpha>2$, since $\mathcal{N}_{\alpha}^{\varphi}=\{(0,0)\}$, $E_{\alpha}^{k}=q$ for every $k \geq 1$, whereas, in the unconstrained problem, by (6.12) we get that if $2<\alpha<\frac{12}{5}$, the discrete motion is not trivial; that is, $E_{\alpha}^{k}=\bigcup_{\|\mathbf{i}\|_{1}=1} q$ (i) for every $k \geq 1$, even though the limit motion is pinned.

Example 46 (a strongly anisotropic norm) We now give, along the lines of Example 37, another example where the discrete minimizers are (degenerate) checkerboard sets and the limit set is one-dimensional, i.e., a linearly growing segment. For this, we construct ad hoc a strongly anisotropic non-absolute norm $\varphi$ such that $\varphi(1,1)<\varphi(1,0)=$ $\varphi(0,1)$. Namely, we consider the symmetric positive definite matrix $\mathbf{A}=\left(a_{i j}\right)$ such that $a_{11}=a_{22}>1, a_{12}<0$ and

$$
\frac{1}{8}<a_{11}+a_{12}<\frac{1}{2}, \quad 2<a_{11}-a_{12} .
$$


Fig. 46 The unit ball of $\varphi$ for $a_{11}=2$ and $a_{12}=-\frac{5}{3}$
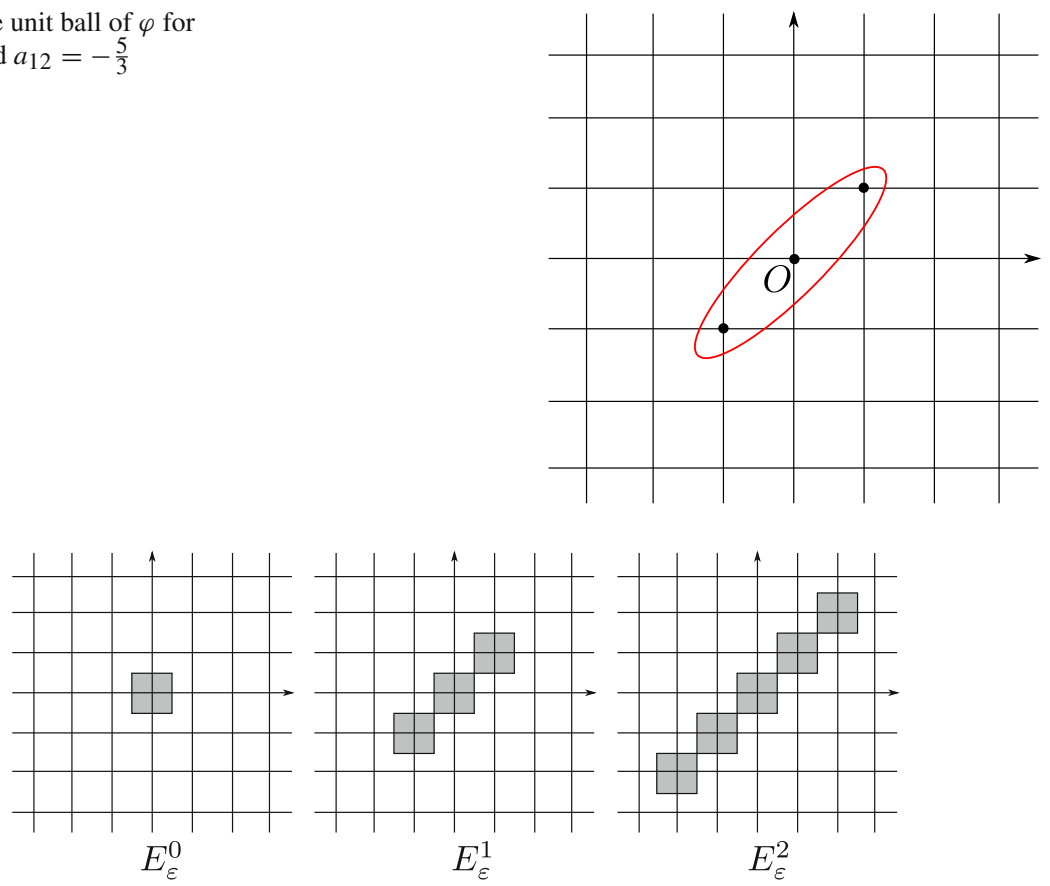

Fig. 47 Some steps of the evolution

Correspondingly, we define the elliptic norm

$$
\varphi(\mathbf{x}):=\sqrt{\mathbf{x}^{t} \mathbf{A} \mathbf{x}}=\sqrt{a_{11}\left(x_{1}^{2}+x_{2}^{2}\right)+2 a_{12} x_{1} x_{2}},
$$

whose unit ball is pictured in Fig. 46.

Assumption (6.13) ensures that $\varphi(1,1)=\sqrt{2\left(a_{11}+a_{12}\right)}<\sqrt{a_{11}}=\varphi(1,0)=$ $\varphi(0,1)$. In addition, we assume that

$$
\frac{4}{\sqrt{a_{11}}}<\alpha \leq \frac{2 \sqrt{2}}{\sqrt{a_{11}+a_{12}}}
$$

In this case, if we let $E_{\alpha}^{0}=q$, the set of centers of the first step is

$$
\mathcal{N}_{\alpha}^{\varphi}=Z\left(E_{\alpha}^{1}\right)=\left\{\mathbf{i} \in \mathbb{Z}^{2}: \varphi(\mathbf{i}) \leq \frac{4}{\alpha}\right\}=\{(-1,-1),(0,0),(1,1)\}
$$

whence, arguing by induction on the step $k$, we infer that

$$
Z\left(E_{\alpha}^{k}\right)=\{(j, j):|j|=0,1, \ldots, k\}, \quad k \geq 1 .
$$


A similar computation as for the proof of Theorem 38 shows that an analogous characterization for $Z\left(E_{\alpha}^{k}\right)$ by means of the Minkowski sum as in (5.39) holds. The polygon $P_{\alpha}^{\varphi}$ here reduces to the line segment $\mathcal{L}$ of length $2 \sqrt{2}$ centered at 0 with slope 1. In Fig. 47, some steps of the discrete evolution are represented. Note that the proof of (6.16)-(6.17) does not require any covering argument in the fashion of Sect. 5.3 or any monotonicity assumption (5.13). The following characterization of the limit evolution immediately follows from the proof of Theorem 39.

Proposition 47 Let $\alpha$ be such that (6.15) holds. Then, there exists a unique minimizing movement of (3.4) $E^{\alpha}(t)=\alpha \mathcal{L} t$ where $\mathcal{L}$ is the line segment above.

\subsection{Further Results and Conjectures}

In this section, we focus on the non-trivial issue of addressing our problem without the monotonicity constraint. If on the one hand in the case of the $\ell^{\infty}$-norm (Example 43), the monotonicity constraint did not play any role, on the other hand in Example 45 we proved that the first step of the unconstrained scheme (6.7) for the $\ell^{1}$-norm can be either an even or an odd checkerboard set. The idea of the proof was to follow the argument of Proposition 27, replacing, when using the $2 \times 2$-square coverings, the family $\mathcal{S}_{e}(E)$ with $\mathcal{S}_{o}(E)$ defined in (6.8) in the case $E \not \supset q$. This approach works for every absolute norm $\varphi$. Therefore, when removing the monotonicity constraint in the minimization scheme, we find the following generalization of Proposition 27.

Proposition 48 Let $\varphi$ be an absolute norm, let $\alpha>0$ be such that $\alpha \notin \Lambda^{\varphi}$, and let $\mathcal{F}_{\alpha}^{\varphi}$ be as in (5.1). Then, the first minimization problem of scheme (6.7) admits the only solutions

$$
E_{\alpha}^{1}=\underset{E \in \mathcal{D}}{\operatorname{argmin}} \mathcal{F}_{\alpha}^{\varphi}(E, q)= \begin{cases}E\left(\mathbb{Z}_{e}^{2} \cap B_{\frac{4}{\alpha}}^{\varphi}\right) \in \mathcal{A}^{e}, & \text { if } q \subset E_{\alpha}^{1}, \\ E\left(\mathbb{Z}_{o}^{2} \cap B_{\frac{4}{\alpha}}^{\varphi}\right) \in \mathcal{A}^{o}, & \text { if } q \not \subset E_{\alpha}^{1} .\end{cases}
$$

At this point, we are forced to depart from Example 45 for the determination of the sets $E_{\alpha}^{k}, k \geq 2$, as the delicate construction of a covering needed in the proof of Proposition 30 strongly relies on the monotonicity constraint on the discrete evolution and thence is no longer enough to infer an analogous result for the subsequent steps of the evolution. The investigation of this issue has therefore to be deferred to further contributions. Anyway, motivated by the previous "positive" examples, we do believe that under suitable assumptions on the norm $\varphi$ and the geometry of the competitors in the minimization problem, one can still infer a (checkerboard) structure result as in Proposition 30 and a characterization by means of Minkowski sums, analogous to that of Theorem 38. Within this scenario, oscillations of the minimizers between checkerboards of different parity, in principle, cannot be excluded. However, energetic considerations suggest that these may occur only for a finite number of steps, depending on $\alpha$ : heuristically, a change of parity at step $k$ involves a variation of the perimeter term of order $k$ which cannot match, for $k$ large, the corresponding increase in the bulk term of order $k^{2}$. In order to see, this we may assume, without loss of generality, 
that $Z\left(E_{\alpha}^{k+1}\right)=(k+1) \mathcal{N}_{\alpha}^{\varphi} \subset \mathbb{Z}_{o}^{2}$ and $Z\left(E_{\alpha}^{k}\right)=k \mathcal{N}_{\alpha}^{\varphi} \subset \mathbb{Z}_{e}^{2}$ for some $k \geq 1$, as an interchanging of the parity of the sets would provide an analogous estimate. Then, by virtue of (2.7)-(2.9), the variation of the energy $\mathcal{F}_{\alpha}^{\varphi}$ from an even checkerboard $E_{\alpha}^{k}$ to the odd one $E_{\alpha}^{k+1}$ is bounded from below by

$$
\begin{aligned}
& -4\left(\# Z\left(E_{\alpha}^{k+1}\right)-\# Z\left(E_{\alpha}^{k}\right)\right)+\alpha \min \{\varphi(1,0), \varphi(0,1)\}\left(\# Z\left(E_{\alpha}^{k}\right)+\# Z\left(E_{\alpha}^{k+1}\right)\right) \\
& =-4 \#\left((k+1) \mathcal{N}_{\alpha}^{\varphi} \cap \mathbb{Z}_{o}^{2}\right)+4 \#\left(k \mathcal{N}_{\alpha}^{\varphi} \cap \mathbb{Z}_{e}^{2}\right)+\alpha\left(\#\left(k \mathcal{N}_{\alpha}^{\varphi} \cap \mathbb{Z}_{e}^{2}\right)+\#\left((k+1) \mathcal{N}_{\alpha}^{\varphi} \cap \mathbb{Z}_{o}^{2}\right)\right) \\
& \geq-4 \#\left((k+1) \mathcal{N}_{\alpha}^{\varphi} \cap \mathbb{Z}_{o}^{2}\right)+4 \#\left(k \mathcal{N}_{\alpha}^{\varphi} \cap \mathbb{Z}_{e}^{2}\right)+\alpha \#\left(k \mathcal{N}_{\alpha}^{\varphi} \cap \mathbb{Z}^{2}\right) \\
& =\alpha\left|\operatorname{conv}\left(\mathcal{N}_{\alpha}^{\varphi}\right)\right| k^{2}+C_{\alpha}^{\prime} k+C_{\alpha}^{\prime \prime} .
\end{aligned}
$$

Thus, there exists $k_{\alpha}:=k(\alpha)$ such that the right-hand side in (6.18) is positive for $k \geq$ $k_{\alpha}$. As a consequence, the change of parity is not energetically favorable (definitely in $k$ ), and we expect either $E_{\alpha}^{k} \in \mathcal{A}_{\text {conv }}^{e}$ or $E_{\alpha}^{k} \in \mathcal{A}_{\text {conv }}^{o}$ for every $k \geq k_{\alpha}$ to hold as a result of iterated Minkowski sums with the even nucleus $\mathcal{N}_{\alpha}^{\varphi}$ of (3.5). In conclusion, since a finite number of oscillations is neglected in the limit, an analogous characterization of the limit evolution as in Theorem 39 holds.

We summarize our conjecture as follows.

Conjecture Under suitable assumptions on $\varphi$ and for suitable values of $\alpha$, the discrete solutions $\left\{E^{k}\right\}$ of scheme (3.5) satisfy

either $Z\left(E_{\alpha}^{k}\right)=\left\{\mathbf{i} \in \mathbb{Z}_{e}^{2}: d^{\varphi}\left(\mathbf{i}, E_{\alpha}^{k-1}\right)<\frac{4}{\alpha}\right\}$ or $Z\left(E_{\alpha}^{k}\right)=\left\{\mathbf{i} \in \mathbb{Z}_{o}^{2}: d^{\varphi}\left(\mathbf{i}, E_{\alpha}^{k-1}\right)<\frac{4}{\alpha}\right\}$.

Moreover, there exists an index $k_{\alpha} \in \mathbb{N}$ such that

$$
Z\left(E_{\alpha}^{k}\right)=Z\left(E_{\alpha}^{k_{\alpha}}\right)+\underbrace{\mathcal{N}_{\alpha}^{\varphi}+\cdots+\mathcal{N}_{\alpha}^{\varphi}}_{\left(k-k_{\alpha}\right) \text {-times }}, \text { for every } k \geq k_{\alpha}
$$

As for the limit evolution, there exists a unique minimizing movement $E:[0,+\infty) \rightarrow$ $X$ for scheme (3.5) defined by $E(t)=v_{\alpha}^{\varphi} t P_{\alpha}^{\varphi}$ for every $t \geq 0$, where $P_{\alpha}^{\varphi}$ and $v_{\alpha}^{\varphi}$ are as in the statement of Theorem 39.

Acknowledgements A. Braides acknowledges the MIUR Excellence Department Project awarded to the Department of Mathematics, University of Rome Tor Vergata, CUP E83C18000100006. G. Scilla has been supported by the Italian Ministry of Education, University and Research through the Project "Variational methods for stationary and evolution problems with singularities and interfaces" (PRIN 2017). A. Tribuzio was supported by the Deutsche Forschungsgemeinschaft (DFG, German Research Foundation) project number RU 2049/1-1 within the SPP 2256 "Variational Methods for Predicting Complex Phenomena in Engineering Structures and Materials."

Funding Open access funding provided by Università degli Studi di Roma Tor Vergata within the CRUICARE Agreement. 
Open Access This article is licensed under a Creative Commons Attribution 4.0 International License, which permits use, sharing, adaptation, distribution and reproduction in any medium or format, as long as you give appropriate credit to the original author(s) and the source, provide a link to the Creative Commons licence, and indicate if changes were made. The images or other third party material in this article are included in the article's Creative Commons licence, unless indicated otherwise in a credit line to the material. If material is not included in the article's Creative Commons licence and your intended use is not permitted by statutory regulation or exceeds the permitted use, you will need to obtain permission directly from the copyright holder. To view a copy of this licence, visit http://creativecommons.org/licenses/by/4.0/.

\section{References}

Alicandro, R., Braides, A., Cicalese, M.: Phase and anti-phase boundaries in binary discrete systems: a variational viewpoint. Netw. Heterog. Media 1, 85-107 (2006)

Ambrosio, L., Fusco, N., Pallara, D.: Functions of Bounded Variations and Free Discontinuity Problems. Oxford University Press, Oxford (2000)

Almgren, F., Taylor, J.E.: Flat flow is motion by crystalline curvature for curves with crystalline energies. J. Diff. Geom. 42(1), 1-22 (1995)

Almgren, F., Taylor, J.E., Wang, L.: Curvature driven flows: a variational approach. SIAM J. Control Optim. 50, 387-438 (1993)

Barki, H., Denis, F., Dupont, F.: Contributing vertices-based Minkowski sum computation of convex polyhedra. Comput. Aided Des. 41(7), 525-538 (2009)

Braides, A.: $\Gamma$-Convergence for Beginners. Oxford University Press, Oxford (2002)

Braides, A.: Local Minimization, Variational Evolution and $\Gamma$-convergence. Lecture Notes in Mathematics, vol. 2094. Springer, Berlin (2013)

Braides, A., Cicalese, M.: Interfaces, modulated phases and textures in lattice systems. Arch. Rat. Mech. Anal. 223, 977-1017 (2017)

Braides, A., Cicalese, M., Yip, N.K.: Crystalline motion of interfaces between patterns. J. Stat. Phys. 165(2), 274-319 (2016)

Braides, A., Gelli, M.S., Novaga, M.: Motion and pinning of discrete interfaces. Arch. Rat. Mech. Anal. 195, 469-498 (2010)

Braides, A., Scilla, G.: Motion of discrete interfaces in periodic media. Interfaces Free Bound. 15, 451-476 (2013)

Braides, A., Scilla, G.: Nucleation and backward motion of discrete interfaces. C. R. Math. Acad. Sci. Paris 351, 803-806 (2013)

Braides, A., Solci, M.: Motion of discrete interfaces through mushy layers. J. Nonlinear Sci. 26, 1031-1053 (2016)

Braides, A., Solci, M.: Geometric Flows on Planar Lattices. Birkhäuser, Basel (2021)

Ciccarese, D.: Influence of temporal and spatial heterogeneity on microbial spatial self-organization. Ph.D. thesis (2020). https://doi.org/10.3929/ethz-b-000401169

Daneri, S., Runa, E.: Exact periodic stripes for minimizers of a local/nonlocal interaction functional in general dimension. Arch. Rat. Mech. Anal. 231, 519-589 (2019)

De Yoreo, J.J., Vekilov, P.G.: Principles of crystal nucleation and growth. Rev. Mineral. Geochem. 54(1), 57-93 (2003)

Gardner, R.J., Gronchi, P., Zong, C.: Sums, projections and sections of lattice sets, and the discrete covariogram. Discrete Comput. Geom. 34, 391-409 (2005)

Giuliani, A., Lebowitz, J.L., Lieb, E.H.: Checkerboards, stripes, and corner energies in spin models with competing interactions. Phys. Rev. B 84, 064205 (2011)

Kalikmanov, V.: Nucleation Theory. Lecture Notes in Physics LNP, Lecture Notes in Physics, vol. 860. Springer, Netherlands (2013)

Lindner, M., Roch, S.: On the integer points in a lattice polytope: $n$-fold Minkowski sum and boundary. Beitr. Algebra Geom. 52, 395-404 (2011)

Marinacci, M., Montrucchio, L.: On concavity and supermodularity. J. Math. Anal. Appl. 344, 642-654 (2008)

Mimura, M., Sakaguchi, H., Matsushita, M.: Reaction-diffusion modelling of bacterial colony patterns. Phys. A Stat. Mech. Its Appl. 282(1-2), 283-303 (2000) 
Murota, K.: Discrete Convex Analysis. SIAM Monographs on Discrete Mathematics and Applications, Society for Industrial and Applied Mathematics (SIAM), Philadelphia, PA (2003)

Pick, G.A.: Geometrisches zur Zahlenlehre. Lotos, Naturwissenschaft. Zeitschr. 19, 311-319 (1899)

Ruf, M.: Motion of discrete interfaces in low-contrast random environments. ESAIM: COCV 24(3), 12751301 (2018)

Scilla, G.: Motion of discrete interfaces in low-contrast periodic media. Netw. Heterog. Media 9, 169-189 (2014)

Scilla, G.: Motion of discrete interfaces on the triangular lattice. Milan J. Math. 88, 315-346 (2020)

Publisher's Note Springer Nature remains neutral with regard to jurisdictional claims in published maps and institutional affiliations. 